\title{
Measurement of Electroweak Parameters from Z Decays into Fermion Pairs
}

\author{
The ALEPH Collaboration
}

\begin{abstract}
We report on the properties of the $\mathrm{Z}$ resonance from $62,500 \mathrm{Z}$ decays into fermion pairs collected with the ALEPH detector at LEP, the Large Electron-Positron storage ring at CERN. We find $M_{\mathrm{Z}}=\left(91.193 \pm 0.016_{\exp } \pm 0.030_{\mathrm{LEP}}\right) \mathrm{GeV}, \Gamma_{\mathrm{Z}}=(2497 \pm$ 31) $\mathrm{MeV}, \sigma_{\text {had }}^{0}=(41.86 \pm 0.66) \mathrm{nb}$, and for the partial widths $\Gamma_{\text {inv }}=(489 \pm 24) \mathrm{MeV}$, $\Gamma_{\text {had }}=(1754 \pm 27) \mathrm{MeV}, \Gamma_{e e}=(85.0 \pm 1.6) \mathrm{MeV}, \Gamma_{\mu \mu}=(80.0 \pm 2.5) \mathrm{MeV}$, and $\Gamma_{\tau \tau}=(81.3 \pm 2.5) \mathrm{MeV}$, all in good agreement with the Standard Model. Assuming lepton universality and using a lepton sample without distinction of the final state we measure $\Gamma_{\ell \ell}=(84.3 \pm 1.3) \mathrm{MeV}$. The forward-backward asymmetry in leptonic decays is used to determine the vector and axial-vector weak coupling constants of leptons, $g_{\mathrm{V}}^{2}\left(M_{\mathrm{Z}}^{2}\right)=(0.12 \pm 0.12) \times 10^{-2}$ and $g_{\mathrm{A}}^{2}\left(M_{\mathrm{Z}}^{2}\right)=0.2528 \pm 0.0040$. The number of light neutrino species is $N_{\nu}=2.91 \pm 0.13$; the electroweak mixing angle is $\sin ^{2} \theta_{\mathrm{W}}\left(M_{\mathrm{Z}}^{2}\right)=$ $0.2291 \pm 0.0040$.
\end{abstract}

Submitted to Zeitschrift für Physik C 


\section{The ALEPH Collaboration}

D. Decamp, B. Deschizeaux, C. Goy, J.-P. Lees, M.-N. Minard

Laboratoire de Physique des Particules (LAPP), IN $P^{3}$-CNRS, 74019 Annecy-le-Vieux Cedex, France

R. Alemany, J.M. Crespo, M. Delfino, E. Fernandez, V. Gaitan, Ll. Garrido, P. Mato, R. Miquel, Ll.M. Mir, S. Orteu, A. Pacheco, J.A. Perlas, E. Tubau

Laboratorio de Fisica de Altas Energias, Universidad Autonoma de Barcelona, 08193 Bellaterra (Barcelona), Spain ${ }^{9}$

M.G. Catanesi, D. Creanza, M. de Palma, A. Farilla, G. Iaselli, G. Maggi, M. Maggi, S. Natali, S. Nuzzo, M. Quattromini, A. Ranieri, G. Raso, F. Romano, F. Ruggieri, G. Selvaggi, L. Silvestris, P. Tempesta, G. Zito

INFN Sezione di Bari e Dipartimento di Fisica dell' Università, 70126 Bari, Italy

Y. Gao, H. Hu, D. Huang, S. Jin, J. Lin, T. Ruan, T. Wang, W. Wu, Y. Xie, D. Xu, R. Xu, J. Zhang, W. Zhao

Institute of High-Energy Physics, Academia Sinica, Beijing, The People's Republic of China ${ }^{10}$

H. Albrecht, W.B. Atwood, F. Bird, E. Blucher, G. Bonvicini, F. Bossi, J. Bourotte, D. Brown, T.H. Burnett, H. Drevermann; F. Dydak, R.W. Forty, C. Grab, R. Hagelberg, S. Haywood, B. Jost, M. Kasemann, G. Kellner, J. Knobloch, A. Lacourt, I. Lehraus, T. Lohse, D. Lüke, A. Marchioro, M. Martinez, J. May2 S. Menary, A. Minten, A. Miotto, J. Nash, P. Palazzi, F. Ranjard, G. Redlinger, A. Roth, J. Rothberg, H. Rotscheidt, W. von Rüden, R. St.Denis, D. Schlatter, M. Takashima, M. Talby, H. Taureg, W. Tejessy, H. Wachsmuth, S. Wasserbaech, S. Wheeler, W. Wiedenmann, W. Witzeling, J. Wotschack

European Laboratory for Particle Physics (CERN), 1211 Geneva 23, Switzerland

Z. Ajaltouni, M. Bardadin-Otwinowska, A. Falvard, R. El Fellous, P. Gay, P. Henrard, J. Jousset, B. Michel, J-C. Montret, D. Pallin, P. Perret, J. Proriol, F. Prulhière

Laboratoire de Physique Corpusculaire, Université Blaise Pascal, IN ${ }^{2} P^{3}$-CNRS, ClermontFerrand, 63177 Aubière, France

J.D. Hansen, J.R. Hansen, P.H. Hansen, R. Møllerud, B.S. Nilsson, G. Petersen

Niels Bohr Institute, 2100 Copenhagen, Denmark ${ }^{11}$

I. Efthymiopoulos, E. Simopoulou, A. Vayaki

Nuclear Research Center Demokritos (NRCD), Athens, Greece

J. Badier, A. Blondel, G. Bonneaud, F. Braems, J.C. Brient, G. Fouque, A. Gamess, R. Guirlet, A. Rosowsky, A. Rougé, M. Rumpf, R. Tanaka, H. Videau, I. Videau ${ }^{1}$

Laboratoire de Physique Nucléaire et des Hautes Energies, Ecole Polytechnique, $I N^{2} P^{3}$. CNRS, 91128 Palaiseau Cedex, France 


\section{D.J. Candlin}

Department of Physics, University of Edinburgh, Edinburgh EH9 3JZ, United Kingdom ${ }^{12}$

G. Parrini

Dipartimento di Fisica, Università di Firenze, INFN Sezione di Firenze, 50125 Firenze, Italy

M. Corden, C. Georgiopoulos, M. Ikeda, J. Lannutti, D. Levinthal, ${ }^{17}$ M. Mermikides, L. Sawyer, G. Stimpfl

Supercomputer Computations Research Institute and Dept. of Physics, Florida State University, Tallahassee, FL 32306, USA $A^{14,15,16}$

A. Antonelli, R. Baldini, G. Bencivenni, G. Bologna, P. Campana, G. Capon, V. Chiarella, B. D'Ettorre-Piazzoli? G. Felici, P. Laurelli, G. Mannocchi? F. Massimo Brancaccio, F. Murtas, G.P. Murtas, G. Nicoletti, M. Pepe-Altarelli, P. Picchi, P. Zografou

Laboratori Nazionali dell'INFN (LNF-INFN), 00044 Frascati, Italy

B. Altoon, O. Boyle, A.W. Halley, I. ten Have, J.L. Hearns, J.G. Lynch, W.T. Morton, C. Raine, J.M. Scarr, K. Smith, A.S. Thompson

Department of Physics and Astronomy, University of Glasgow, Glasgow G12 8QQ, United Kingdom ${ }^{12}$

B. Brandl, O. Braun, R. Geiges, C. Geweniger, P. Hanke, V. Hepp, E.E. Kluge, Y. Maumary, M. Panter, A. Putzer, B. Rensch, A. Stahl, K. Tittel, M. Wunsch

Institut für Hochenergiephysik, Universität Heidelberg, 6900 Heidelberg, Fed. Rep. of Germany ${ }^{18}$

A.T. Belk, R. Beuselinck, D.M. Binnie, W. Cameron, M. Cattaneo, P.J. Dornan, S. Dugeay, A.M. Greene, J.F. Hassard, S.J. Patton, J.K. Sedgbeer, G. Taylor, I.R. Tomalin, A.G. Wright

Department of Physics, Imperial College, London SW7 2BZ, United Kingdom ${ }^{12}$

P. Girtler, E. Kneringer, D. Kuhn, G. Rudolph

Institut für Experimentalphysik, Universität Innsbruck, 6020 Innsbruck, Austria ${ }^{20}$

C.K. Bowdery, T.J. Brodbeck, A.J. Finch, F. Foster, G. Hughes, N.R. Keemer, M. Nuttall, B.S. Rowlingson, T. Sloan, S.W. Snow

Department of Physics, University of Lancaster, Lancaster LA1 4YB, United Kingdom ${ }^{12}$

T. Barczewski, L.A.T. Bauerdick, K. Kleinknecht, B. Renk, S. Roehn, H.-G. Sander, M. Schmelling, F. Steeg

Institut für Physik, Universität Mainz, 6500 Mainz, Fed. Rep. of Germany ${ }^{18}$ 
J-P. Albanese, J-J. Aubert, C. Benchouk, V. Bernard, A. Bonissent, D. Courvoisier, F. Etienne, S. Papalexiou, P. Payre, B. Pietrzyk, Z. Qian

Centre de Physique des Particules, Faculté des Sciences de Luminy, IN ${ }^{2} P^{3}$-CNRS, 13288 Marseille, France

W. Blum, P. Cattaneo, G. Cowan, B. Dehning, H. Dietl, M. Fernandez-Bosman, M. Frank, A. Jahn, E. Lange, G. Lütjens, G. Lutz, W. Männer, H-G. Moser, Y. Pan, R. Richter, A.S. Schwarz, R. Settles, U. Stiegler, U. Stierlin, J. Thomas

Max-Planck-Institut für Physik und Astrophysik, Werner-Heisenberg-Institut für Physik, 8000 München, Fed. Rep. of Germany ${ }^{18}$

V. Bertin, G. de Bouard, J. Boucrot, O. Callot, X. Chen, A. Cordier, M. Davier, G. Ganis, J.F. Grivaz, Ph. Heusse, P. Janot, V. Journé, D.W. Kim, ${ }^{21}$ J. Lefrançois, A.-M. Lutz, J.-J. Veillet, Z. Zhang, F. Zomer

Laboratoire de l'Accéléateur Linéaire, Université de Paris-Sud, $I N^{2} P^{3}$-CNRS, 91405 Orsay Cedex, France

S.R. Amendolia, G. Bagliesi, G. Batignani, L. Bosisio, U. Bottigli, C. Bradaschia, M.A. Ciocci, I. Ferrante, F. Fidecaro, L. Foàํㅕㄹ. E. Focardi, F. Forti, A. Giassi, M.A. Giorgi, F. Ligabue, A. Lusiani, E.B. Mannelli, P.S. Marrocchesi, A. Messineo, F. Palla, G. Sanguinetti, J. Steinberger, R. Tenchini, G. Tonelli, G. Triggiani

Dipartimento di Fisica dell'Università, INFN Sezione di Pisa, e Scuola Normale Superiore, 56010 Pisa, Italy

J.M. Carter, M.G. Green, P.V. March, T. Medcalf, M.R. Saich, J.A. Strong, R.M. Thomas, T. Wildish

Department of Physics, Royal Holloway \& Bedford New College, University of London, Surrey TW20 OEX, United Kingdom ${ }^{12}$

D.R. Botterill, R.W. Clifft, T.R. Edgecock, M. Edwards, S.M. Fisher, J. Harvey, T.J. Jones, P.R. Norton, D.P. Salmon, J.C. Thompson

Particle Physics Dept., Rutherford Appleton Laboratory, Chilton, Didcot, OXON OX11 $O Q X$, United Kingdom ${ }^{12}$

B. Bloch-Devaux, P. Colas, C. Klopfenstein, E. Lançon, E. Locci, S. Loucatos, L. Mirabito, E. Monnier, P. Perez, F. Perrier, J. Rander, J.-F. Renardy, A. Roussarie, J.-P. Schuller

Département de Physique des Particules Élémentaires, CEN-Saclay, 91191 Gif-sur-Yvette Cedex, France ${ }^{19}$

J.G. Ashman, C.N. Booth, F. Combley, M. Dinsdale, J. Martin, D. Parker, L.F. Thompson Department of Physics, University of Sheffield, Sheffield S3 7RH, United Kingdom ${ }^{12}$

S. Brandt, H. Burkhardt, C. Grupen, ${ }^{1}$ H. Meinhard, E. Neugebauer, U. Schäfer, H. Seywerd Fachbereich Physik, Universität Siegen, 5900 Siegen, Fed. Rep. of Germany ${ }^{18}$ 
G. Apollinari, G. Giannini, B. Gobbo, F. Liello, E. Milotti, L. Rolandi ${ }^{1}$

Dipartimento di Fisica, Università di Trieste e INFN Sezione di Trieste, 34127 Trieste, Italy

L. Bellantoni, J.F. Boudreau, D. Cinabro, J.S. Conway, D.F. Cowen, A.J. DeWeerd, Z. Feng, D.P.S. Ferguson, Y.S. Gao, J.L. Harton, J. Hilgart, J.E. Jacobsen, R.C. Jared, R.P. Johnson, B.W. LeClaire, Y.B. Pan, T. Parker, J.R. Pater, Y. Saadi, V. Sharma, J.A. Wear, F.V. Weber, Sau Lan Wu, G. Zobernig

Department of Physics, University of Wisconsin, Madison, WI 53706, USA ${ }^{13}$

(Submitted to Zeitschrift für Physik C)

\footnotetext{
${ }^{1}$ Now at CERN.

${ }^{2}$ Permanent address: DESY, Hamburg, Fed. Rep. of Germany.

${ }^{3}$ On leave of absence from SLAC, Stanford, CA 94309, USA.

${ }^{4}$ On leave of absence from University of Washington, Seattle, WA 98195, USA.

${ }^{5}$ Also Centre de Physique des Particules, Faculté des Sciences, Marseille, France

${ }^{\circ}$ Also Istituto di Fisica Generale, Università di Torino, Torino, Italy.

${ }^{7}$ Also Istituto di Cosmo-Geofisica del C.N.R., Torino, Italy.

${ }^{8}$ Permanent address: LBL, Berkeley, CA 94720 , USA.

${ }^{9}$ Supported by CAICYT, Spain.

${ }^{10}$ Supported by the National Science Foundation of China.

${ }^{11}$ Supported by the Danish Natural Science Research Council.

${ }^{12}$ Supported by the UK Science and Engineering Research Council.

${ }^{13}$ Supported by the US Department of Energy, contract DE-AC02-76ER00881.

${ }^{14}$ Supported by the US Department of Energy, contract DE-FG05-87ER40319.

${ }^{15}$ Supported by the NSF, contract PHY-8451274.

${ }^{16}$ Supported by the US Department of Energy, contract DE-FC0S-85ER250000.

${ }^{17}$ Supported by SLOAN fellowship, contract BR 2703 .

${ }^{18}$ Supported by the Bundesministerium für Forschung und Technologie, Fed. Rep. of Germany.

${ }^{19}$ Supported by the Institut de Recherche Fondamentale du C.E.A..

${ }^{20}$ Supported by Fonds zur Förderung der wisseuschaftlichen Forschung, Austria.

${ }^{21}$ Supported by Non Directed Research Fund, Korea Res. Found., 1989.
} 


\section{Contents}

1 Introduction $\quad 6$

2 The Detector 6

$\begin{array}{llc}3 & \text { The } \text { Triggers } & 8\end{array}$

4 The Determination of Luminosity 9

4.1 The Luminosity Detector and Trigger . . . . . . . . . . . . . . . . 9

4.2 The Fiducial Definition . . . . . . . . . . . . . . . . 11

4.3 The Luminosity Event Selection . . . . . . . . . . . . . . . 11

4.4 The Bhabha Cross Section and Systematic Error . . . . . . . . . 13

5 The Hadronic Z Decays $\quad 16$

5.1 The Selection based on Charged Tracks . . . . . . . . . . . . . . 16

5.2 The Calorimetric Selection . . . . . . . . . . . . . . . 18

5.3 The Hadronic Cross Section . . . . . . . . . . . . . . . . . 21

6 The Leptonic Z Decays 22

6.1 The $Z \rightarrow e^{+} e^{-}$Cross Section $\ldots \ldots \ldots \ldots$

6.2 The $Z \rightarrow \mu^{+} \mu^{-}$Cross Section . . . . . . . . . . . 27

6.3 The $\mathrm{Z} \rightarrow \tau^{+} \tau^{-}$Cross Section . . . . . . . . . . . . 29

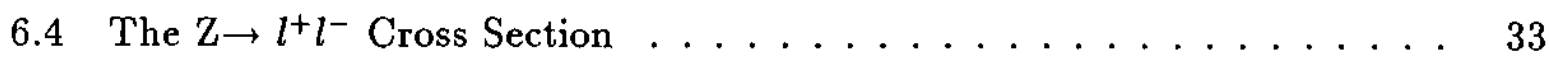

7 The Z Resonance Parameters $\quad 36$

8 The Determination of Vector and Axial-vector Couplings 40

9 Standard Model Interpretation $\quad 43$

9.1 The Measurement of the Number of Light Neutrino Species . . . . . . . 44

9.2 The Determination of $\sin ^{2} \theta_{\mathrm{w}}\left(M_{\mathrm{z}}^{2}\right) \ldots \ldots \ldots \ldots \ldots$

9.3 Comparison with Other Experiments: $\sin ^{2} \theta_{\mathrm{W}}\left(M_{\mathrm{Z}}^{2}\right)$ and Limits on the Top Mass ......................... 46

10 Conclusion $\quad 47$

11 Acknowledgements $\quad 48$ 


\section{Introduction}

In the summer of 1989 , with the beginning of the operation of the Large Electron Positron storage ring (LEP) at CERN and of the SLC at SLAC, a large number of Z particles became available for precise measurements of electroweak parameters [1,2]. The most important result has been the accurate determination of the peak cross-section, with the conclusion that the number of light neutrino species, $N_{\nu}$, into which the Z decays is three. In addition the measurement of the $\mathrm{Z}$ mass with a relative precision of $3 \times 10^{-4}$ has fixed a fundamental constant of the electroweak theory.

The availability of higher luminosity makes possible tests of the Standard Model to a higher level of accuracy than was previously possible in either' neutrino scattering or $e^{+} e^{-}$or $\mathrm{p} \overline{\mathrm{p}}$ collider experiments. Besides the total $\mathrm{Z}$ cross section and width, precise measurements of the leptonic $\mathrm{Z}$ decay widths can be used to test, with high sensitivity, the predictions of the electroweak theory. Furthermore, the measured ratio

$R=\Gamma_{\text {had }} / \Gamma_{\ell \ell}$ of the hadronic to leptonic partial width can be used in a less modeldependent determination of $N_{\nu}$.

In this paper we present results on the $\mathrm{Z}$ resonance parameters from decays into hadrons and charged lepton pairs, based on two data samples recorded in ALEPH in 1989 and up to the end of May 1990. An integrated luminosity of $2.5 \mathrm{pb}^{-1}$ has been obtained corresponding to 56,000 hadronic and 6,500 leptonic decays in an energy scan around the $\mathrm{Z}$ resonance peak ${ }^{1}$. The two data sets, which are about equal in size, have been analysed together. Event-selection procedures and systematic errors are discussed in detail. In addition to more precise results on the $\mathrm{Z}$ mass, partial widths, total width, and $N_{\nu}$, the leptonic decay asymmetries, which permit a direct determination of the leptonic weak vector coupling, are also presented.

\section{The Detector}

The ALEPH detector is described in detail in our technical publication [3]. Displays of computer-reconstructed events from the four visible decay modes of the $\mathrm{Z}$ into hadrons, $e^{+} e^{-}, \mu^{+} \mu^{-}$, and $\tau^{+} \tau^{-}$pairs are shown in Fig. 1. The detector components relevant for the measurements presented in this paper are:

- The Inner Tracking Chamber (ITC), an 8-layer cylindrical drift chamber with sense wires parallel to the beam axis from $13 \mathrm{~cm}$ to $29 \mathrm{~cm}$ in radius. Particles with polar angles from $14^{\circ}$ to $166^{\circ}$ traverse all 8 layers.

- The large cylindrical Time Projection Chamber (TPC), extending from an inner radius of $31 \mathrm{~cm}$ to an outer radius of $180 \mathrm{~cm}$ over a length of $4.4 \mathrm{~m}$. Up to 21 space points are recorded for particles with polar angles from $37^{\circ}$ to $143^{\circ}$. Requiring at least 4 points, tracks are reconstructed down to $15^{\circ}$.

- The Electromagnetic Calorimeter (ECAL), a lead/wire-chamber sandwich covering the polar angle range from $11^{\circ}$ to $169^{\circ}$. The cathode readout is subdivided into a total of 73,728 projective towers. Each tower of less than $1^{\circ} \times 1^{\circ}$ solid angle

\footnotetext{
${ }^{1}$ In 1989 the LEP centre-of-mass energies were $88.278,89.284,90.282,91.034,91.283,91.530,92.280$, $93.287,94.278$, and $95.036 \mathrm{GeV}$; in $1990 \mathrm{LEP}$ has been operating at $\sim 60 \mathrm{MeV}$ lower.
} 

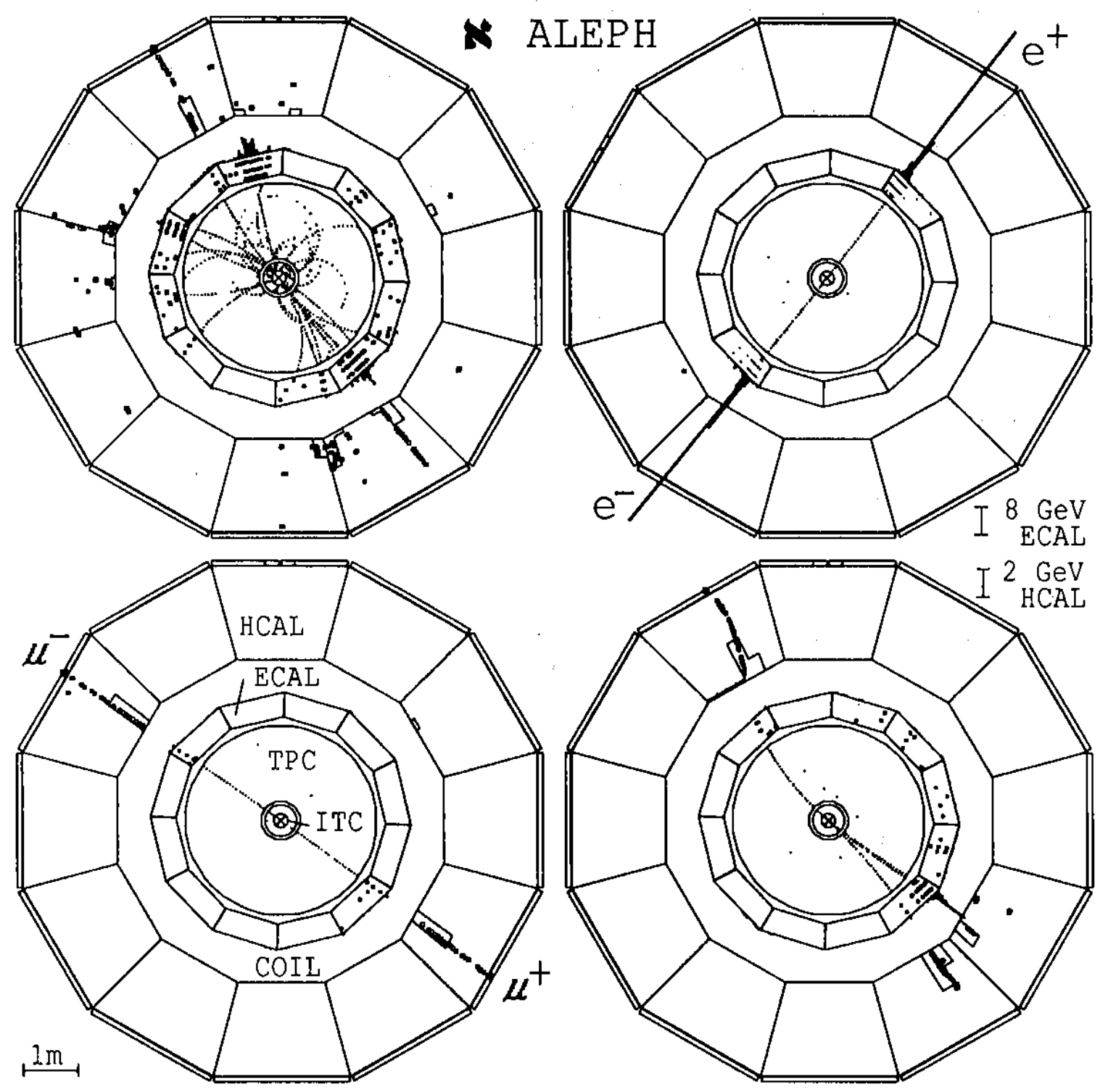

Figure 1: Display of Z decays into hadrons, and $e^{+} e^{-}, \mu^{+} \mu^{-}$and $\tau^{+} \tau^{-}$pairs. The hits in the ITC and TPC, the pulse-height histograms of the ECAL and HCAL, and the digital pattern of HCAL tubes are shown. 
is read out in three storeys of 4,9 and 9 radiation lengths providing detailed energy measurements in both azimuth and polar angles. The signals from the 45 wire planes of each module are also read out, allowing the measurement of the longitudinal shower development in a module. The timing of the pulse-height signals with respect to the beam crossing is recorded.

- The superconducting solenoid providing a magnetic field of $1.5 \mathrm{~T}$.

- The Hadron Calorimeter (HCAL), consisting of 23 layers of streamer tubes interleaved in the iron of the magnet return yoke, segmented in 4800 projective towers. It covers a polar angle range from $6^{\circ}$ to $174^{\circ}$. Digital signals from each of the tubes are also read out. The modules are rotated in azimuth by $\sim 2^{\circ}$ with respect to the electromagnetic calorimeter so that inactive zones do not align in the two calorimeters.

- The luminosity detectors, described in Section 4.

\section{The Triggers}

Full details about the ALEPH trigger system may be found elsewhere [3] but for the Z decays two kinds of triggers are used :

- A total energy trigger derived from the ECAL by summing separately the signals from the wire planes of the 12 modules in the barrel and the 12 modules in each of the two end-caps. An energy above $6.6 \mathrm{GeV}$ in the barrel, $3.8 \mathrm{GeV}$ in either end-cap, or $1.5 \mathrm{GeV}$ in both end-caps is required by this trigger.

- Two charged-particle triggers requiring a track candidate in the ITC in coincidence with a wire signal from a group of ECAL or HCAL modules forming a trigger segment. There are 12 segments in azimuth in the barrel and 6 segments in each of the end-caps. Only the segments close in azimuth to the track are allowed in the coincidence: three segments $\left(90^{\circ}\right)$ in the barrel and two segments $\left(120^{\circ}\right)$ in the end-caps. A track candidate must have hits in at least five out of the eight ITC layers, and the associated energy in an ECAL segment must exceed $1.3 \mathrm{GeV}$, or the particle must penetrate the ECAL and at least $30 \mathrm{~cm}$ to $90 \mathrm{~cm}$ of iron in the HCAL, depending on the polar angle. The ITC trigger is fully efficient for tracks with transverse momenta above $1 \mathrm{GeV}$ and angles with respect to the beam axis of more than $15^{\circ}$.

The trigger efficiency depends on the characteristics of the selected event sample and is determined in each case from the data using redundant trigger information.

The basic trigger for hadronic events is the total ECAL energy trigger. Its performance is monitored by the penetrating charged-particle trigger, which is entirely independent, since it is based on the ITC and HCAL and covers more than $90 \%$ of the events. One finds a trigger efficiency of $(99.96 \pm 0.02) \%$ for the total energy trigger for hadronic events.

For the $e^{+} e^{-}$final state the total energy trigger is compared with the charged-particle trigger requiring an energy deposit in the ECAL, which is far below the cuts used in the event selection. No evidence of any inefficiency is observed. 


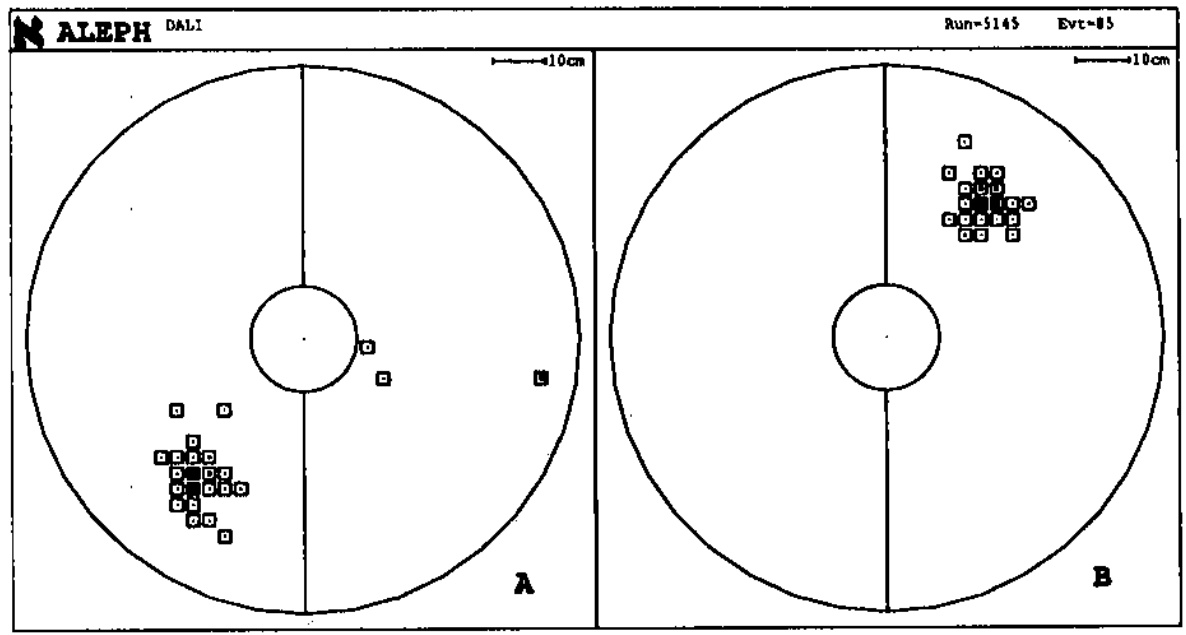

Figure 2: Display of a luminosity event; the energy deposits of the electron and positron in the hit LCAL towers are shown.

For the other leptonic final states the charged-particle triggers are used. Since these triggers are segmented in azimuth, the two leptons of a pair trigger independently. Possible correlations due to insensitive regions between the calorimeter modules are found to be negligible at the present level of statistical accuracy. Moreover, the bending of the magnetic field prevents back-to-back high-momentum tracks both pointing to insensitive regions between modules. The single-lepton trigger efficiency is measured from the observed events and is used to calculate the overall efficiency for lepton pairs. The trigger conditions are observed to be stable with the exception of the first three months of data taking during which the performance of the HCAL was improving.

The triggers for luminosity events are described in the following section.

\section{The Determination of Luminosity}

\subsection{The Luminosity Detector and Trigger}

The luminosity monitor consists of a calorimeter (LCAL) and a small-angle tracking device (SATR). During this first running period SATR was only used for verification of the LCAL alignment and to optimize the beam conditions, since SATR is very sensitive to synchrotron radiation and thus presents a valuable background monitor. The luminosity is determined from small-angle Bhabha events showering in the LCAL, which consists of 38 layers of lead sheets and proportional-wire chambers. The design is optimized to minimize the systematic error from the uncertainty of the positioning of the sensitive elements. LCAL is read out in projective towers, 768 at either side of the interaction point. A display of a typical event is shown in Fig. 2, and the tower layout of one half of one module is shown in Fig. 3.

Each tower is read out in 3 storeys of $4.8,10.6$ and 9.25 radiation lengths in depth. 
Figure 3: One half of one module of the LCAL, showing the tower structure and the fiducial region (outlined in bold).

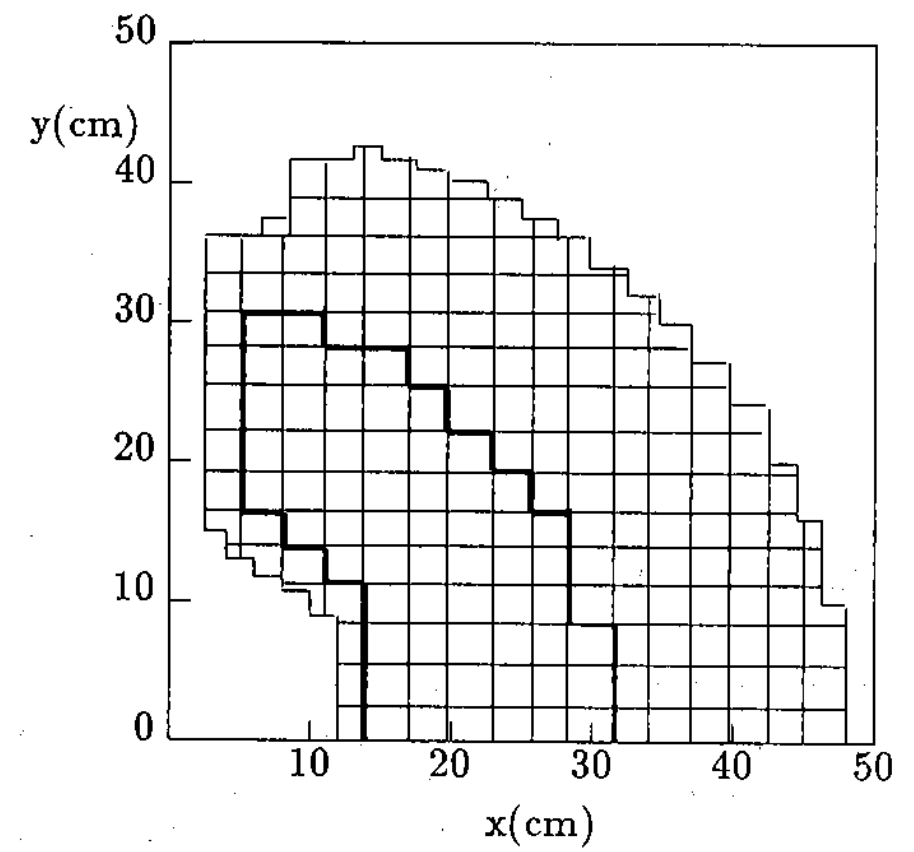

There are in total 4 semicylindrical modules, two each at $\pm 262 \mathrm{~cm}$ from the nominal centre of ALEPH, covering polar angles from 42 to $160 \mathrm{mrad}$. Within each module, the mechanical precision in the relative position of the towers is $120 \mu \mathrm{m}$. The uncertainty on the inner radius due to the relative positioning of the two modules is $140 \mu \mathrm{m}$. The energy resolution at $45 \mathrm{GeV}$ is about $3 \%$, in agreement with test-beam measurements. The tower-to-tower response is uniform within $\pm 2 \%$, and the spatial resolution of the shower position is $1.4 \mathrm{~mm}$ in the horizontal and vertical directions.

Three triggers, based on the analog sum of pulse heights in the towers," are used in the luminosity determination:

- A luminosity trigger, which requires a coincidence between the electron and the positron sides with more than $20 \mathrm{GeV}$ on one and $16 \mathrm{GeV}$ on the other.

- A high-energy single-arm trigger, requiring more than $31 \mathrm{GeV}$ on either side. This trigger permits the efficiency determination of the luminosity trigger.

- Two prescaled single-arm triggers with one requiring more than $20 \mathrm{GeV}$ and the other more than $16 \mathrm{GeV}$ on either side. These enable the determination of the background due to random coincidences caused by off-momentum electrons and positrons.

The luminosity trigger efficiency varies slightly with time; on average it is $99.7 \%$, but it is always larger than $98 \%$. 


\subsection{The Fiducial Definition}

To minimize the systematic uncertainty in the luminosity measurement, a fiducial region is defined on just one calorimeter with the choice of the calorimeter alternating from event to event. The centre of the shower produced by the electron or the positron is required to be in the fiducial region. This fiducial region excludes towers at the inner edges of the calorimeter (see Fig. 3) and an outer region, approximately for polar angles larger than $110 \mathrm{mrad}$, where the calorimeter is shadowed by dense interior support structures, e.g. support rings for the TPC and cabling for the ITC. The cut on the outer region results in a loss of only $\sim 15 \%$ of the luminosity events, but substantially reduces the tails of the energy distributions which are otherwise difficult to simulate.

Events with showers that are well contained inside the fiducial boundary are kept. If the tower with the largest energy, which is typically more than $60 \%$ of the total, occurs next to, but on either side of the fiducial boundary, then the energies in the first two storeys of this tower and its eight nearest neighbors are used to determine if the centroid of the shower is inside or outside the region. In the case where the boundary is straight, the fiducial condition is simply that the sum of the energies of the three towers just inside the boundary be greater than the sum for the adjacent three towers just outside the boundary. If the tower with the largest energy occurs next to a corner of the fiducial boundary, then the condition is applied separately to both the horizontal and vertical sets of the three adjacent towers. In this case the sum of the energies for the row or column that is mostly inside the boundary is required to be greater than that for the row and column that is outside. These cuts are applied only on the fiducial side and there are no such requirements on the other side.

The requirement of fiducial side alternation from event to event, ensures that the acceptance is independent, to first order, of transverse and longitudinal displacements of the collision point, and of small angular changes of the beam direction. The consequent corrections are negligible, for example, a horizontal beam shift of $3 \mathrm{~mm}$ would result in a loss of less than $1 \times 10^{-3}$ in the accepted number of luminosity events. The horizontal and vertical vertex distribution at the nominal beam crossing, an example of which is shown in Fig. 4 , is measured with a precision of $\sim 200 \mu \mathrm{m}$ for each LEP fill on the basis of the shower positions on the two sides. The r.m.s. spread of $1.2 \mathrm{~mm}$ is consistent with the LCAL point resolution of $1.4 \mathrm{~mm}$, taking into account the effect of bremsstrahlung.

\subsection{The Luminosity Event Selection}

The Bhabha event selection is based on the energy deposited by the electron and by the positron in the corresponding calorimeters. Neighbouring towers containing more than $50 \mathrm{MeV}$ are joined into clusters, giving the energy and position of the shower. The energy on each side is required to be larger than $44 \%$ of the beam energy and, to reduce beam-related background, the sum of the two energies must be larger than $60 \%$ of the centre-of-mass energy. Figure 5 a shows the correlation of the two energies together with these cuts.

To further reduce the beam-related background, the difference in the azimuth, $\Delta \phi$, between the $e^{+}$and $e^{-}$is required to be larger than $170^{\circ}$. Figure $6 \mathrm{a}$ shows the $\Delta \phi$ 

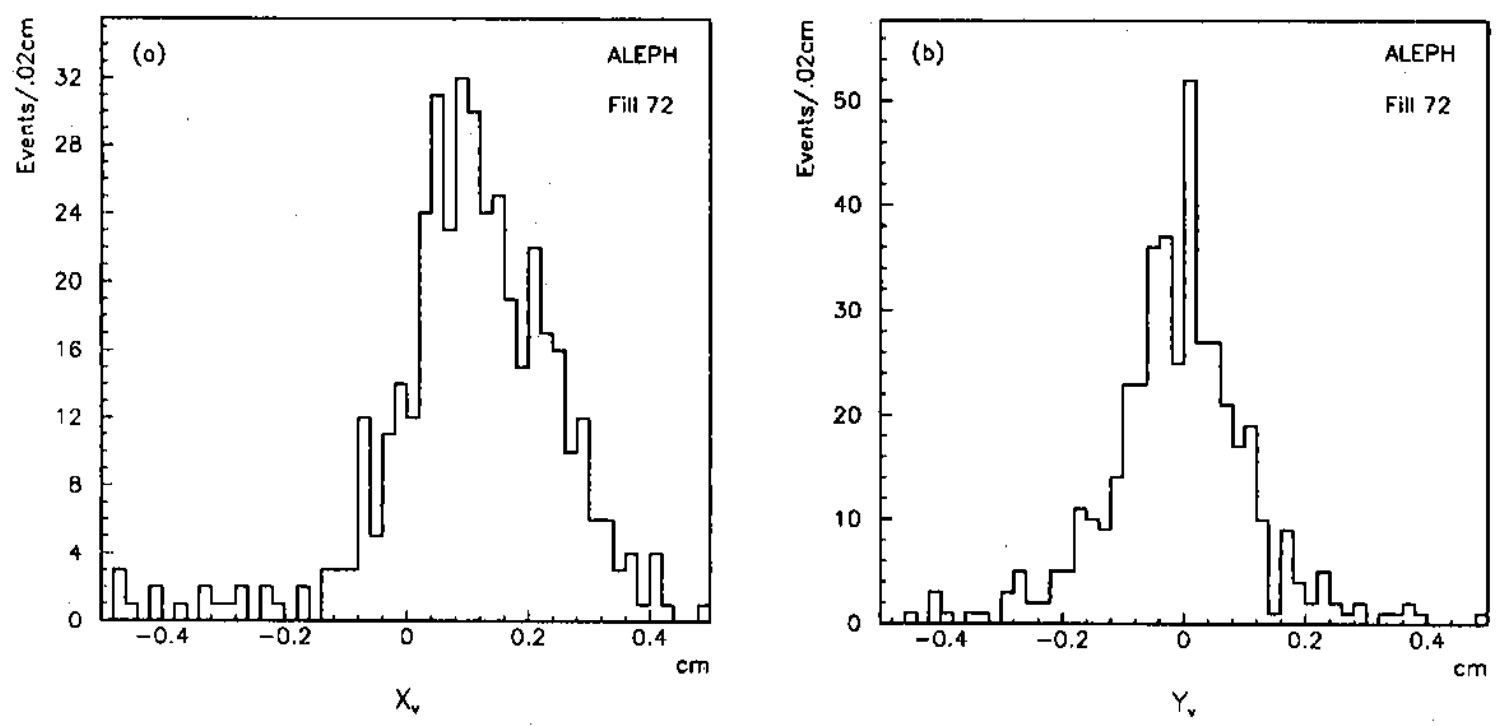

Figure 4: Vertex position at beam crossing as reconstructed from Bhabha events. a) Horizontal, b) vertical.
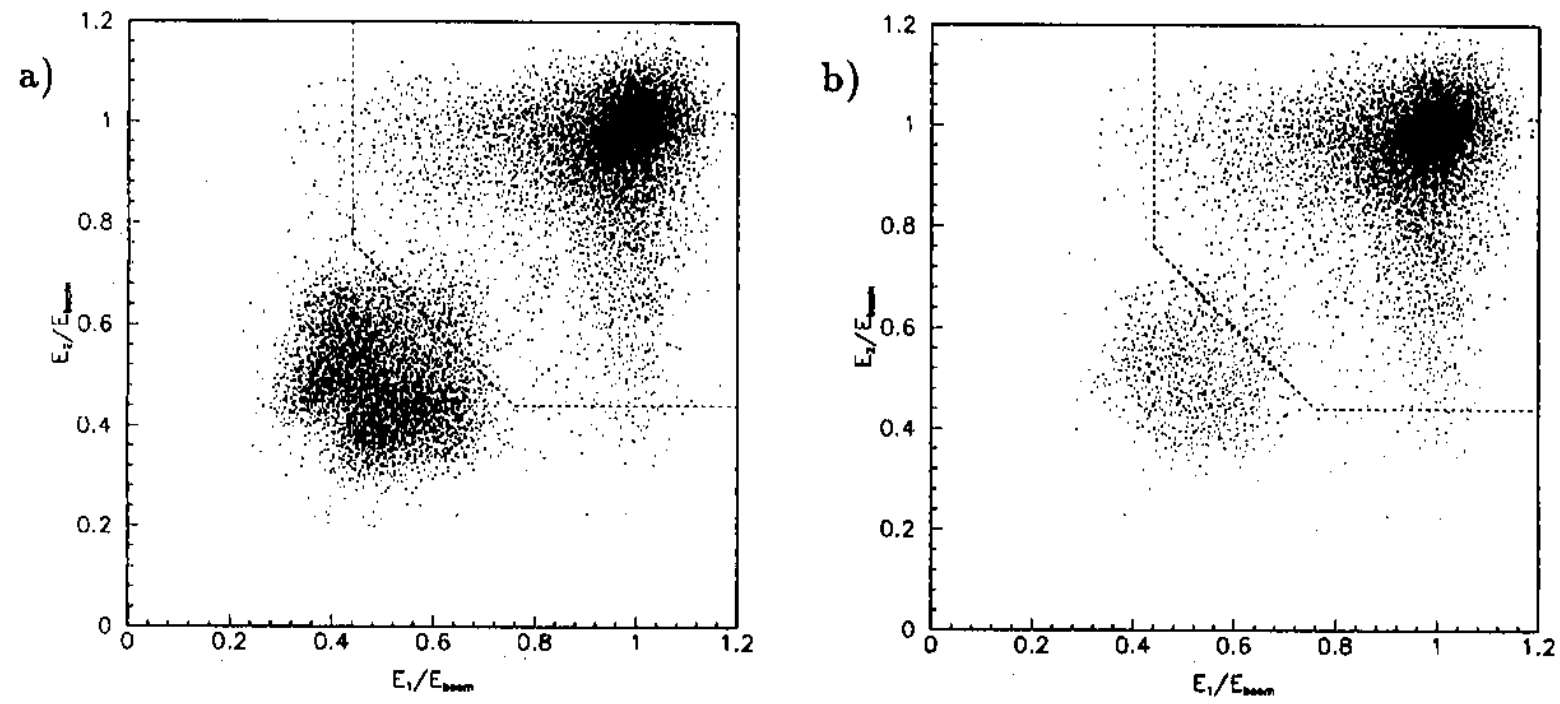

Figure 5: a) Shower energy in the luminosity calorimeter on the fiducial side plotted against that in the non-fiducial side. The dotted line shows the event selection cuts. b) The same but for events with $\Delta \phi>170^{\circ}$. 
Table 1: Summary of systematic errors in the luminosity measurement. Values listed are fractions of the luminosity.

\begin{tabular}{|l|l|}
\hline Position of pad towers within a module and between two halves & 0.003 \\
Energy resolution and cell-to-cell calibration & 0.001 \\
Energy scale & 0.002 \\
Beam parameters & 0.001 \\
Inadequacy of simulation & 0.009 \\
Statistics of simulation & 0.004 \\
\hline Total experimental uncertainty & 0.011 \\
\hline Uncertainty in theory & 0.007 \\
\hline
\end{tabular}

distribution, both for data and simulation, for events accepted by the other cuts after correction for the beam position and magnetic deflection.

The reduction of the background by this $\Delta \phi$ cut is clearly seen in the energy correlation plot of Fig. $5 \mathrm{~b}$; approximately 70,000 luminosity triggers are retained after the $\Delta \phi$ cut alone, with $88.4 \%$ passing also the energy cut. The $\Delta \phi$ distribution also permits an estimate of the background due to random coincidences on the two sides resulting from single, off-momentum particles. Studies using single-arm triggers to create fake coincidence events show that the background events populate the $0^{\circ}<\Delta \phi<20^{\circ}$ and $160^{\circ}<\Delta \phi<180^{\circ}$ regions roughly equally and with like energy distributions. Therefore, for coincidence triggers, the number of events in the region $0^{\circ}<\Delta \phi<10^{\circ}$ which pass the energy cuts is an estimate of background in the signal region. The background is very small, at a level of $\sim 0.4 \%$, as can be seen in Fig. $6 \mathrm{a}$ and in the Bhabha energy distribution in Fig. 6b. The inadequacy of the simulation, seen in Fig. 6b, at high energy does not affect the systematic error on the event selection. Finally, Fig. $6 \mathrm{c}$ shows that the polar-angle distribution of accepted events on the fiducial side is in excellent agreement with the simulation.

\subsection{The Bhabha Cross Section and Systematic Error}

The effective cross section is calculated using an event generator that includes first-order radiative corrections [4] and a full simulation of the detector. The events are generated at $91.0 \mathrm{GeV}$ centre-of-mass energy with a $\mathrm{Z}$ mass of $91.0 \mathrm{GeV}$. The corresponding cross section for our acceptance is found to be $\left(26.59 \pm 0.29_{\exp } \pm 0.20_{\text {theory }}\right) \mathrm{nb}$ [5]. For other energies this cross section is multiplied by a factor of $(91 \mathrm{GeV})^{2} / s$ and corrected for small $(<0.6 \%)$ electroweak interference effects [4]. The hadronic vacuum polarization has been included [6]; for the small momentum transfers involved its uncertainty is of the order of $10^{-4}$ and therefore negligible. The contribution from $e^{+} e^{-} \rightarrow \gamma \gamma$ has been estimated to be $5 \times 10^{-4}$ of the Bhabha cross section for our acceptance and is therefore negligible. Our estimate of various systematic errors is given in Table 1. 

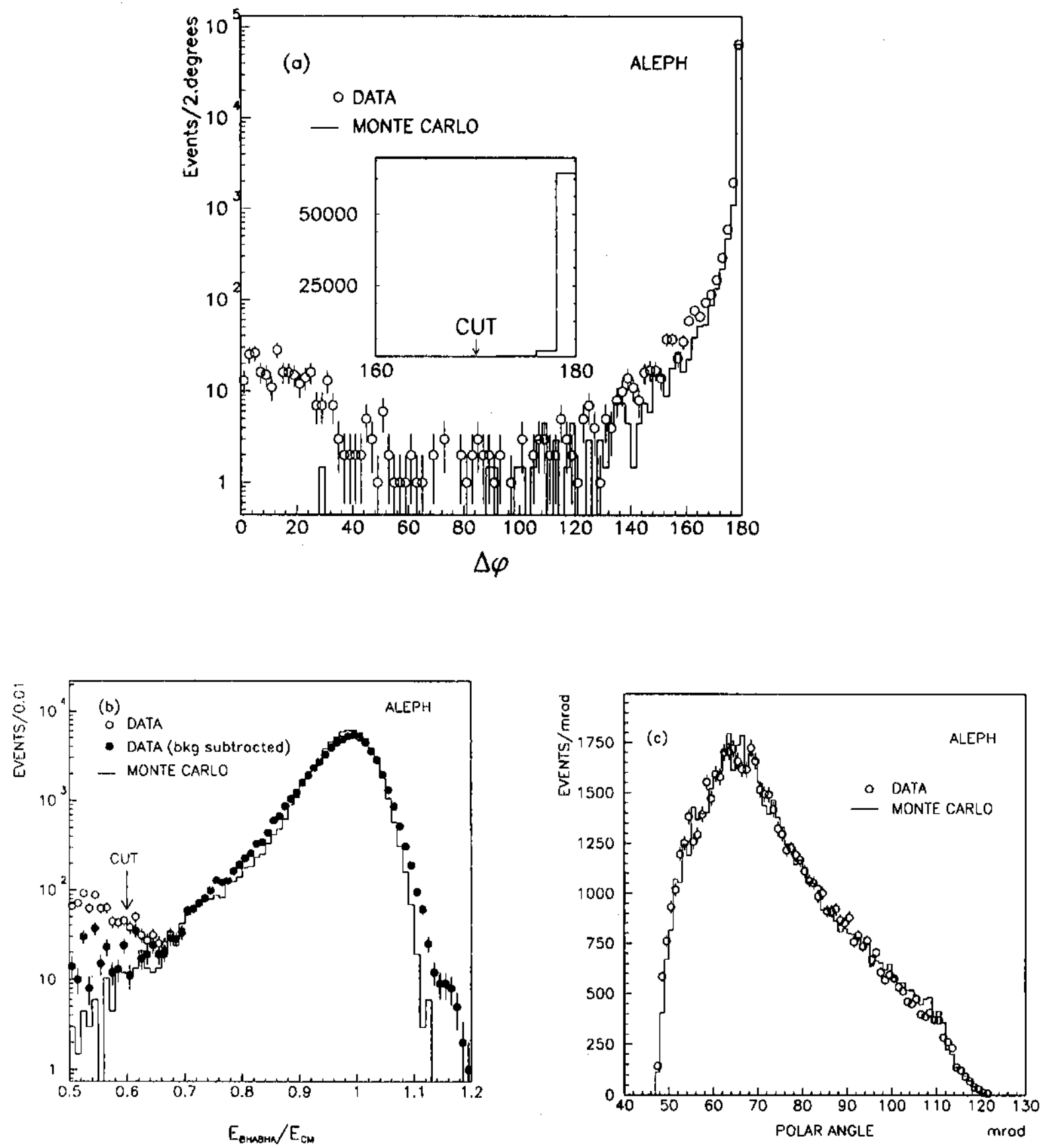

Figure 6: a) Distribution of the difference in the azimuthal angle of the $e^{+}$and $e^{-}$ side after correction for the magnetic deflection. b) Distribution of the total Bhabha energy relative to the centre-of-mass energy before and after background subtraction. c) Distribution of the polar angle of accepted events on the fiducial side. 
The experimental exror on the Bhabha cross section results from Monte Carlo statistics and the effect of uncertainties in the simulation, calibration, and positioning of the calorimeter.

The second error on the Bhabha cross section is an estimate of the uncertainty in the radiative corrections [7]. Within our cuts the cross section is not very sensitive to radiative corrections; the difference between lowest-order and first-order calculations is $+2.5 \%$. The systematic error introduced by neglecting higher orders is expected to be smaller than this difference; and an error of $0.7 \%$ is assumed. The total systematic error attributed to the luminosity measurement is $1.3 \%$.

Table 2: Acceptance changes for different shower energy cuts. The data are background subtracted. The cuts used in this analysis are $E_{1_{12}}>0.44 E_{\text {beam }}$ and $E_{1}+E_{2}>0.6 E_{\text {beam }}$.

\begin{tabular}{|c|c|c|c|}
\hline Cut description & $\begin{array}{c}\text { Data } \\
(\%)\end{array}$ & $\begin{array}{c}\text { Simulation } \\
(\%)\end{array}$ & $\begin{array}{c}\text { Difference } \\
(\%)\end{array}$ \\
\hline$E_{1,2}>0.33 E_{\text {beam }}, E_{1}+E_{2}>0.60 \sqrt{s}$ & +0.18 & +0.09 & +0.09 \\
$E_{1,2}>0.55 E_{\text {beam }}, E_{1}+E_{2}>0.60 \sqrt{s}$ & -0.54 & -0.45 & -0.09 \\
$E_{1,2}>0.44 E_{\text {beam }}, E_{1}+E_{2}>0.55 \sqrt{s}$ & +0.10 & +0.05 & +0.05 \\
$E_{1,2}>0.44 E_{\text {beam }}, E_{1}+E_{2}>0.65 \sqrt{s}$ & -0.15 & -0.07 & -0.08 \\
$\sqrt{E_{1}^{2}+E_{2}^{2}} / E_{\text {beam }}>0.95$ & +0.05 & -0.03 & +0.08 \\
\hline
\end{tabular}

Several checks are made to confirm the systematic error of the luminosity measurement:

- The accuracy of the geometrical event selection is checked by using different acceptance criteria. In one such variation the selection is based on the energy deposited in the second calorimetric storey and compared to that using the first storey. In a sub-sample of data, 21,506 events are selected on the basis of the second storey and 21,480 on the basis of the first. A second variant, based on the selection of the tower with the highest energy in the shower, yielded 21,513 events. The same comparison made with a sample of Monte Carlo events gave 46,279 first storey events, 46,477 second storey events, and 46,406 highest energy tower events. The largest discrepancy, $0.3 \%$, is consistent with the $0.3 \%$ systematic error given in Table 1 .

- Another selection has been made where the inner fiducial boundary is displaced by one tower width to a larger radius. The number of accepted events was found to be $0.7015 \pm 0.0030$ with respect to that obtained by the standard selection; the Monte Carlo expectation is 0.7020 . This indicates that the simulation of the tower boundaries is adequate.

- The sensitivity to the energy cuts has been studied. Both the cuts on single-arm energy as well as on the energy sum have been varied. In addition, a circular cut in the correlation plot of the two LCAL energies $\left(\sqrt{E_{1}^{2}+E_{2}^{2}}>0.95 E_{\text {beam }}\right)$ has been applied. The results are given in Table 2 . The number of accepted events never changed by more than $0.5 \%$ in either data or simulation. The discrepancies between data and simulation are of the order of $0.1 \%$. 
- Although the background is small, a second estimate of background using the singlearm prescaled triggers has been studied. These triggers are combined into artificial double-arm events. The number of such combinations passing the selection cuts with $\Delta \phi<90^{\circ}$ is normalized to the number of events in the region $\Delta \phi<90^{\circ}$ of the actual coincidences. The estimate of background in the signal region is identical with the one obtained by the standard method.

\section{The Hadronic Z Decays}

Events from hadronic $Z$ decays are selected in two independent ways, leading to a reduced systematic error for the average of the two methods. One selection is based on charged tracks only, the other on calorimetric energy. Both methods have an efficiency very close to unity, and the two data samples used in the analysis overlap to the extent of $95 \%$.

\subsection{The Selection based on Charged Tracks}

The selection based on charged tracks, using few cuts, requires at least 5 charged tracks in the TPC. The sum of the energies of the tracks (assuming the pion mass) is required to be larger than $10 \%$ of the centre-of-mass energy. The tracks must have a polar angle above $18.2^{\circ}$, which ensures that at least $6 \mathrm{TPC}$ pad rows are traversed, and must have more than 4 reconstructed coordinates. The distance of closest approach of the tracks to the origin must be less than $10 \mathrm{~cm}$ along the beam direction and $2 \mathrm{~cm}$ transverse to it. After these cuts 56,336 events are retained.

Distributions in global event parameters, such as the charged multiplicity, the polar angle of the sphericity axis and the charged energy are shown to be in excellent agreement with simulation in Fig. 7. Individual track parameters are also in good agreement [8] with hadronization models [9].

The selection efficiency for hadronic $\mathrm{Z}$ decays is calculated from the Monte Carlo simulation to be $(97.5 \pm 0.6) \%$. The error corresponds to the effect of a change of the energy cut by $20 \%$ and the charged multiplicity cut by \pm 1 . Uncertainties in the hadronization models are reduced to a very small level by using the measured sphericity distribution for the acceptance calculation.

The background from $\tau^{+} \tau^{-}$events is estimated to be $0.18 \%$, and is subtracted. Background from beam-gas interactions is estimated from the number of events passing all selection cuts except for the requirement of the vertex position along the beam. It is found to be negligible.

Below the cut of $0.10 \sqrt{s}$ in the total charged energy, the background from twophoton processes, $e^{+} e^{-} \rightarrow e^{+} e^{-}+$hadrons, is dominant (see Fig 7a). The background in the accepted sample has been estimated by exploiting the different centre-of-mass energy dependences of resonant and non-resonant contributions. Figure 8 shows the correlation between the cross sections in the low-energy interval $0.10 \sqrt{s}$ to $0.15 \sqrt{s}$, where a background from two photon processes is expected, and for energies above 

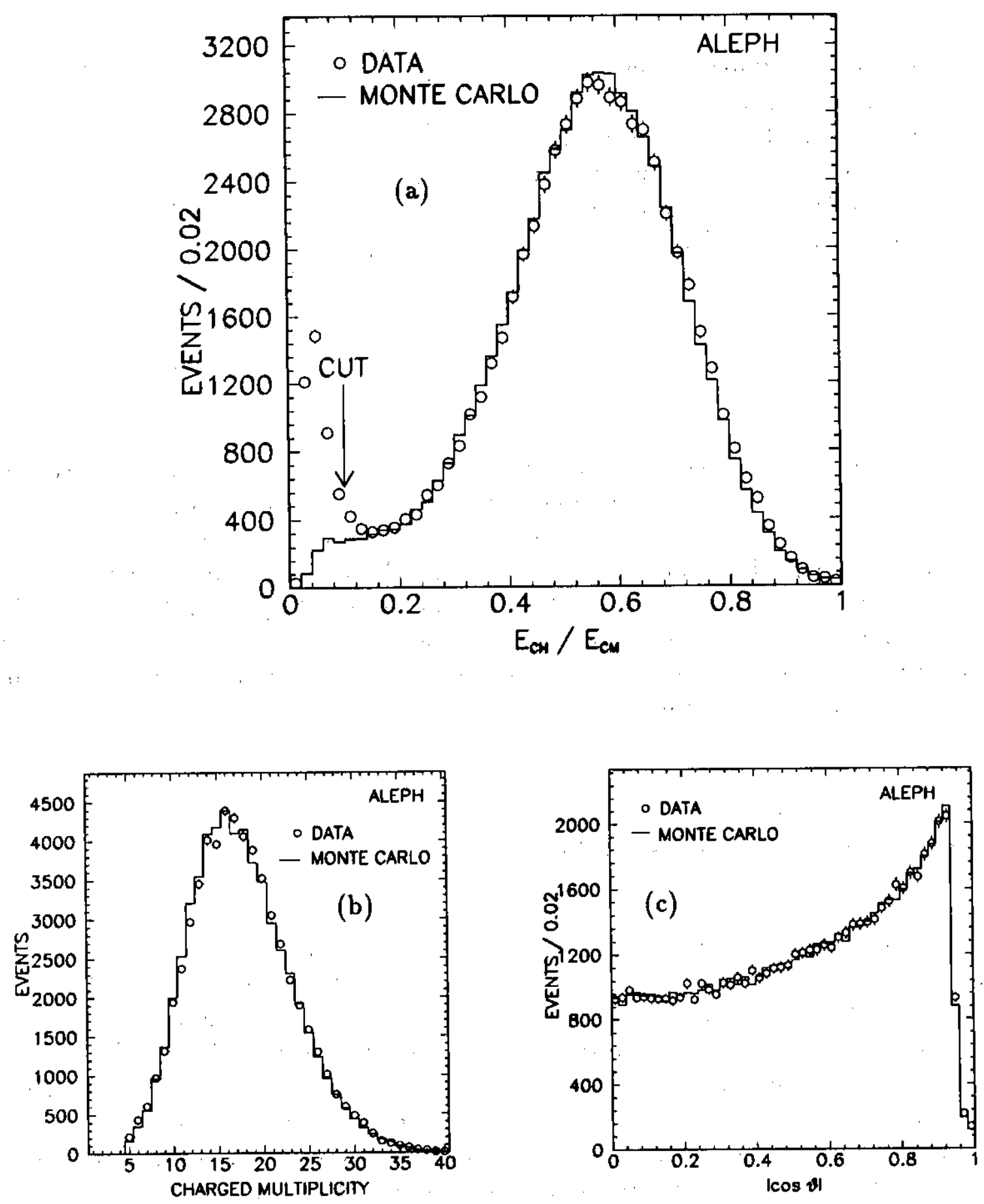

Figure 7: Distributions for events with the track-based selection. a) Total charged energy divided by the centre-of-mass energy. b) Multiplicity of charged tracks. c) Absolute value of the cosine of the angle of the sphericity axis with repect to the beam direction. 
Figure 8: Hadronic cross sections in the charged energy interval $(0.10,0.15) \times \sqrt{s}$ as a function of those in the interval $(0.3,1.0) \times \sqrt{s}$ at different centre-of-mass energies.

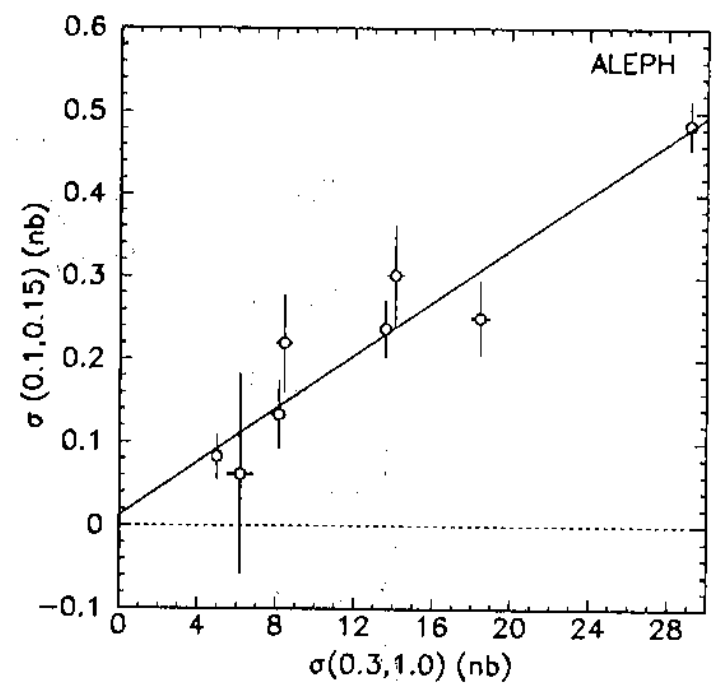

$0.3 \sqrt{s}$, where it is expected to be negligible. A straight-line fit yields a two-photon background in the lower energy interval and this is extrapolated to the full acceptance with a simulation of two-photon processes. This gives $(20 \pm 35) \mathrm{pb}$ for the 1989 data and $(50 \pm 26) \mathrm{pb}$ for 1990 ; these contributions have been subtracted from the cross sections. The excess of data over Monte Carlo beween $0.1 \sqrt{s}$ and $0.15 \sqrt{s}$ (see Fig $7 \mathrm{a}$ ) is not fully explained by the fitted non-resonant cross section. The additional excess (corresponding to about $0.1 \%$ of the peak cross section) ,howewer, appears to be non resonant and is not subtracted.

\subsection{The Calorimetric Selection}

The other, independent, event selection uses calorimetric data to select hadronic $\mathrm{Z}$ decays. The acceptance is slightly larger compared to the track based selection and data collected when the TPC was not operational are recuperated. Events are required to have ECAL energy measured with the wire readout of at least $7 \mathrm{GeV}$ in the barrel or $1.5 \mathrm{GeV}$ in each end-cap, as well as a combined ECAL and HCAL energy of at least $20 \%$ of the centre-of-mass energy. These requirements, as will be seen, reduce twophoton event rates to a negligible level. A time window of $\pm 100 \mathrm{~ns}$, measured on ECAL signals, removes the bulk of cosmic-ray background. After these cuts 75,430 events are preselected of which 52,008 have 5 or more tracks and are accepted. The other 23,422 events with less than 5 tracks contain backgrounds which are removed by additional cuts:

- Bhabha events are removed on the basis of large but local energy deposition in the ECAL: the two most energetic ECAL clusters of the event are required to be less than 35 and $25 \mathrm{GeV}$, respectively. Two particular angular regions, the area 
of overlap between barrel and end-cap, where energy resolution is degraded, and the low-angle area, where part of the event may be lost due to ECAL geometrical acceptance, need more care. For these regions, the additional requirement is made that the summed energy of the two main ECAL clusters is less than $84 \%$ of the total calorimetric energy. In total 12,536 Bhabha candidates are rejected. For a sub-sample of the rejected events the angular distribution is shown in Fig. 9. It compares well with the theoretical expectation for Bhabha events.

- $\mathrm{Z} \rightarrow \tau^{+} \tau^{-}$events are removed by their characteristic collimated back-to-back jet structure. The calorimetrically measured thrust value must be less than 0.996 and the minor value of the energy tensor must be larger than 0.03. After these cuts the remaining $\tau$-pair background, estimated with simulated data, is small $(0.5 \%)$ and a correction is made to the cross section. In total $2,098 \tau^{+} \tau^{-}$candidates are rejected.

- $\mathrm{Z} \rightarrow \mu^{+} \mu^{-}$decays, which pass the total energy trigger threshold because they have a radiative photon, are eliminated by rejecting events with exactly two tracks, each with 4 or more associated hits in the outer 10 layers of the HCAL. This removes 121 events. The remaining $\mu$-pair background is negligible.

- Remaining cosmic-ray background in time with the beam is removed in the following way. Events with exactly one track are rejected unless this track does projects to the vertex cylinder, which is $2 \mathrm{~cm}$ in radius and $\pm 20 \mathrm{~cm}$ along the beam direction. Events are also rejected if there are less than two ECAL clusters of more than $3 \mathrm{GeV}$. These cuts remove 1181 events; $32 \pm 11$ cosmic-ray events are calculated to remain on the basis of the properties of the events outside the timing window.

The final calorimetrically selected hadronic sample contains 59,494 events.

Events from two-photon processes are suppressed very efficiently by the preselection. Possible remaining background has been estimated using the events with between 20 and $35 \mathrm{GeV}$ of calorimetric energy, where the two-photon events would be concentrated. The same method is used as for the track-based selection and at most $9 \mathrm{pb}$ remain in the sample.

The remaining Bhabha background can be studied in a similar way. As it is concentrated at small angles, an enriched sample was chosen as those events with less than 5 tracks and $|\cos \theta|>0.95$ and compared with the full sample as a function of the beam energy. Here, the background term consists not only of the non-resonant photon exchange, but also of the $\mathrm{Z}$ exchange and the interference between them. An estimated background of $9 \mathrm{pb}$ has been removed in the cross section calculations.

The selection efficiency, calculated by Monte Carlo simulation, is $(9.9 .2 \pm 0.2) \%$ for hadronic decays. The total uncertainty in the detection efficiency, including that due to the backgrounds discussed above, is estimated to be $0.6 \%$. The excess of $4.4 \%$ in the calorimetrically selected events with respect to the track selected. events is in part due to the different acceptances for hadronic events (1.9\%), and the rest due to data taken when the TPC was not turned on. Detailed comparison of data with at least 5 tracks finds agreement within $0.3 \%$. 


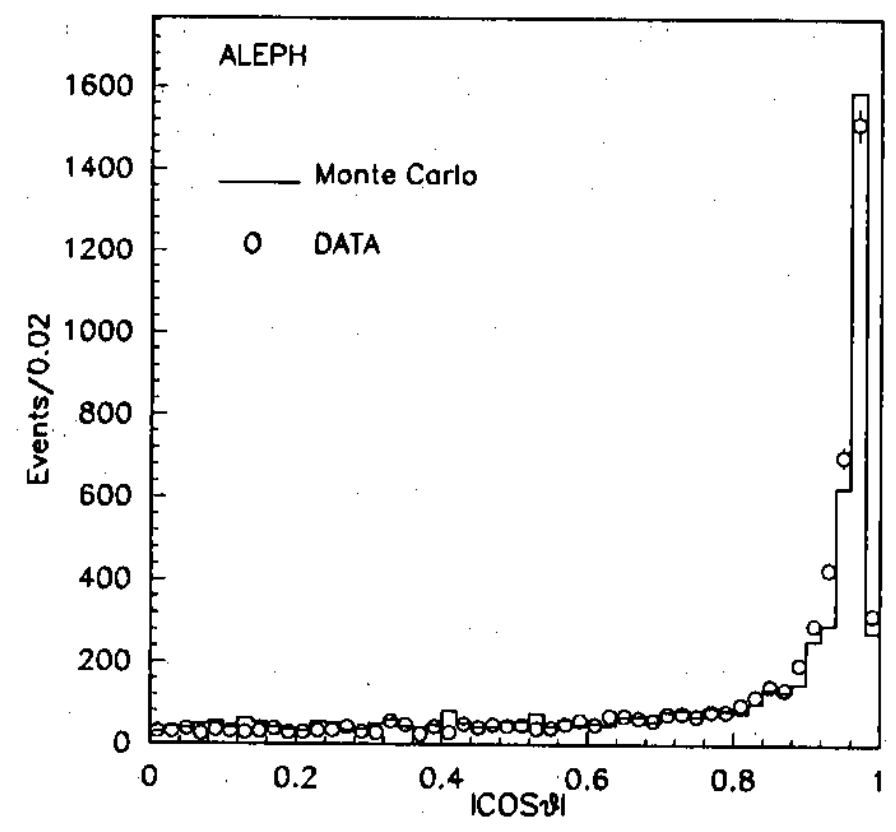

Figure 9: Angular distribution of the thrust axis for selected Bhabha events. The peak at very small angles is due to t-channel photon exchange. The histogram shows the Monte Carlo expectation for the same luminosity.
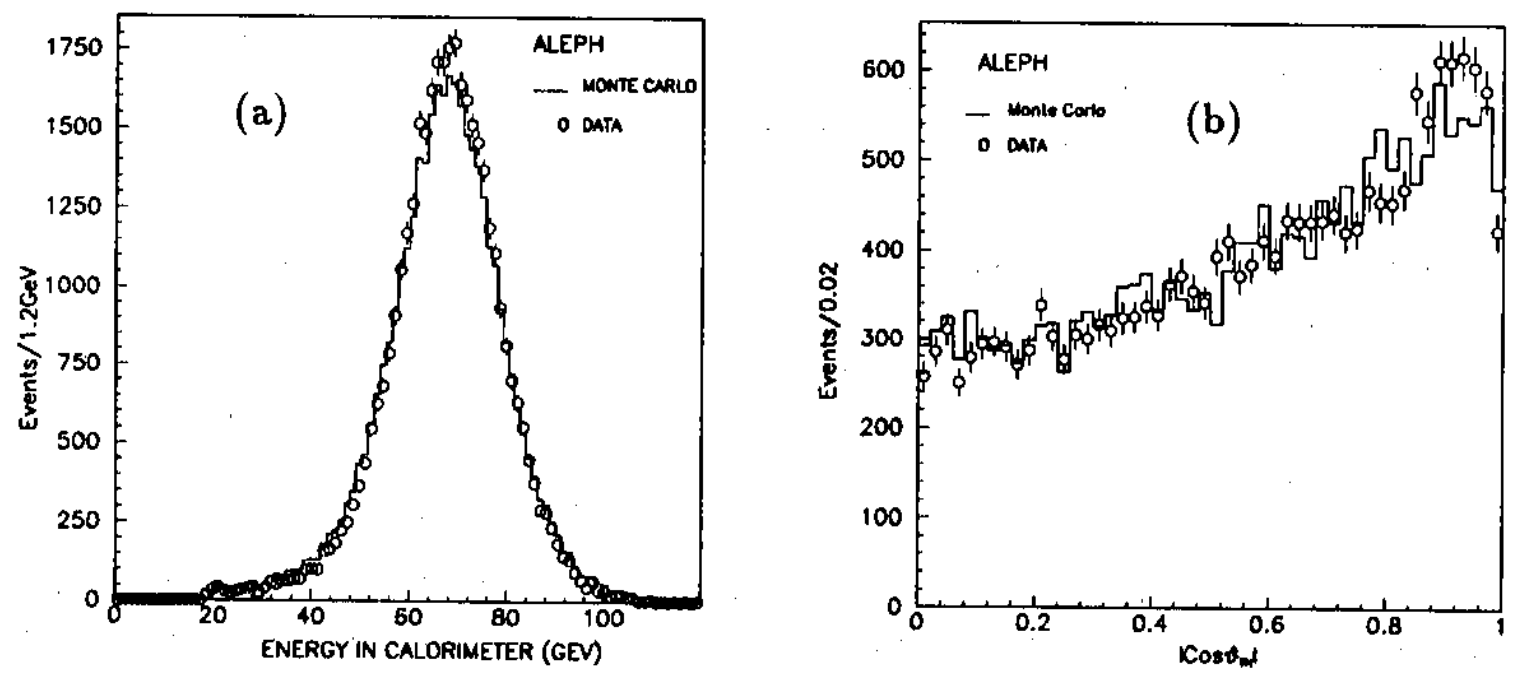

Figure 10: Distributions of calorimetrically selected events. a) Total calorimetric energy. b) Absolute value of the cosine of the angle of the thrust axis. 
Some event distributions are compared to the simulation in Fig. 10. Figure 10a shows the distribution in the total calorimetric energy and Fig. 10b the distribution in the angle of the calorimetrically determined thrust axis. Agreement between data and simulation is good.

\subsection{The Hadronic Cross Section}

Table 3: Event numbers, integrated luminosities, and cross sections for $\mathrm{Z} \rightarrow$ hadrons as a function of centre-of-mass energy. The overall systematic error of $\pm 1.4 \%$ is not included.

\begin{tabular}{|c|rrr|rrr|r|}
\hline$\sqrt{s}$ & \multicolumn{3}{|c|}{ Track selection } & \multicolumn{3}{c|}{ Calorimeter selection } & Average \\
$(\mathrm{GeV})$ & $\mathcal{L}^{\text {int }}$ & \multicolumn{1}{|c|}{$N_{\text {had }}$} & $\begin{array}{l}\sigma_{\text {had }} \\
\left(\mathrm{nb}^{-1}\right)\end{array}$ & \multicolumn{1}{c|}{$\mathcal{L}^{\text {int }}$} & $N_{\text {had }}$ & $\begin{array}{c}\sigma_{\text {had }} \\
\left(\mathrm{nb}^{-1}\right)\end{array}$ & $\sigma_{\text {had }}$ \\
\hline 88.227 & $118.0 \pm 2.0$ & 555 & $4.77 \pm 0.22$ & $118.0 \pm 2.0$ & 556 & $4.72 \pm 0.22$ & $4.74 \pm 0.22$ \\
88.278 & $109.3 \pm 2.0$ & 487 & $4.55 \pm 0.22$ & $125.0 \pm 2.1$ & 575 & $4.73 \pm 0.21$ & $4.63 \pm 0.22$ \\
89.225 & $100.0 \pm 1.9$ & 803 & $8.17 \pm 0.33$ & $100.0 \pm 1.9$ & 817 & $8.19 \pm 0.33$ & $8.18 \pm 0.33$ \\
89.284 & $63.7 \pm 1.5$ & 573 & $9.21 \pm 0.44$ & $84.5 \pm 1.8$ & 771 & $9.23 \pm 0.38$ & $9.22 \pm 0.41$ \\
90.225 & $81.6 \pm 1.7$ & 1492 & $18.68 \pm 0.63$ & $81.5 \pm 1.7$ & 1512 & $18.61 \pm 0.62$ & $18.65 \pm 0.63$ \\
90.282 & $123.9 \pm 2.2$ & 2396 & $19.79 \pm 0.53$ & $126.2 \pm 2.2$ & 2461 & $19.66 \pm 0.52$ & $19.74 \pm 0.52$ \\
91.034 & $182.9 \pm 2.6$ & 5271 & $29.51 \pm 0.59$ & $207.9 \pm 2.8$ & 6070 & $29.43 \pm 0.55$ & $29.47 \pm 0.57$ \\
91.224 & $828.2 \pm 5.6$ & 25202 & $31.11 \pm 0.29$ & $825.1 \pm 5.6$ & 25685 & $31.29 \pm 0.29$ & $31.20 \pm 0.29$ \\
91.283 & $213.6 \pm 2.8$ & 6350 & $30.44 \pm 0.56$ & $224.3 \pm 2.9$ & 6810 & $30.65 \pm 0.55$ & $30.55 \pm 0.55$ \\
91.530 & $175.4 \pm 2.0$ & 5169 & $30.18 \pm 0.62$ & $196.6 \pm 2.7$ & 5780 & $29.61 \pm 0.57$ & $29.89 \pm 0.60$ \\
92.221 & $49.5 \pm 1.4$ & 1111 & $22.95 \pm 0.94$ & $49.5 \pm 1.4$ & 1138 & $23.09 \pm 0.94$ & $23.02 \pm 0.94$ \\
92.280 & $133.6 \pm 2.3$ & 2800 & $21.45 \pm 0.56$ & $137.1 \pm 2.3$ & 2900 & $21.33 \pm 0.54$ & $21.38 \pm 0.55$ \\
92.565 & $8.7 \pm 0.6$ & 130 & $15.31 \pm 1.72$ & $8.8 \pm 0.6$ & 138 & $15.81 \pm 1.73$ & $15.55 \pm 1.73$ \\
93.222 & $139.1 \pm 2.4$ & 1695 & $12.43 \pm 0.37$ & $139.1 \pm 2.4$ & 1724 & $12.34 \pm 0.37$ & $12.39 \pm 0.37$ \\
93.287 & $87.4 \pm 1.9$ & 1141 & $13.37 \pm 0.50$ & $98.1 \pm 2.0$ & 1283 & $13.21 \pm 0.46$ & $13.29 \pm 0.48$ \\
94.219 & $54.7 \pm 1.5$ & 443 & $8.24 \pm 0.45$ & $54.7 \pm 1.5$ & 447 & $8.19 \pm 0.45$ & $8.22 \pm 0.45$ \\
94.278 & $76.4 \pm 1.8$ & 623 & $8.34 \pm 0.39$ & $81.5 \pm 1.8$ & 669 & $8.32 \pm 0.38$ & $8.33 \pm 0.38$ \\
95.036 & $16.4 \pm 0.8$ & 95 & $5.93 \pm 0.69$ & $27.4 \pm 1.1$ & 158 & $5.93 \pm 0.53$ & $5.93 \pm 0.61$ \\
\hline
\end{tabular}

Data have been obtained in a scan around the $\mathrm{Z}$ resonance peak. The number of hadronic $Z$ decays, the luminosity, and the hadronic cross sections are given in Table 3 for the two event-selection methods.

Although the calorimetric selection uses track counting to some extent for background rejection, it is largely independent of the efficiency of the TPC. The dominant sources of systematic error, the geometrical acceptance for the track selection and the background rejection for the calorimetric selection, are thus independent and the systematic error on the average of the two cross section is reduced.

The systematic errors, not included in Table 3 , are $1.3 \%$ for the luminosity measurement, $0.6 \%$ for each hadronic $\mathrm{Z}$ selection, and $0.4 \%$ for the arithmetic mean of the two selections. 
Adding these errors in quadrature, the overall systematic error for the combined hadron cross section is $1.4 \%$. In the following analysis, the mean cross sections of the two event selections, as given in Table 3 , are used.

\section{The Leptonic Z Decays}

The analysis of the leptonic decays of the $\mathrm{Z}$ is performed in two different ways. The first method uses the particle-identification capabilities of ALEPH to isolate separate samples of $e^{+} e^{-}, \mu^{+} \mu^{-}$, and $\tau^{+} \tau^{-}$pairs. In the second, the charged leptonic decays are selected using only the tracking information, and thus all three types of leptons are included without any attempt to distinguish between them. This method is not affected by the systematic errors due to final-state identification and, assuming lepton universality, the leptonic cross section can be measured with smaller systematic error.

In order to extract the forward-backward asymmetry, $A_{\mathrm{FB}}$, the angular distribution at each centre-of-mass energy is fitted by the function

$$
\frac{\mathrm{d} \sigma}{\mathrm{d} \cos \theta^{*}}=C \cdot\left(1+\cos ^{2} \theta^{*}+\frac{8}{3} A_{\mathrm{FB}} \cos \theta^{*}\right) \cdot F\left(\cos \theta^{*}\right)
$$

using a maximum likelihood method. Here $C$ is a normalization constant and $F\left(\cos \theta^{*}\right)$, relevant only for the Bhabha channel, describes the effect of the t-channel exchange. The centre-of-mass scattering angle between the incoming $e^{-}$and the outgoing fermion, $\theta^{*}$, is defined as:

$$
\cos \theta^{*}=\cos \frac{1}{2}\left(\theta_{1}+\pi-\theta_{2}\right) / \cos \frac{1}{2}\left(\theta_{1}-\pi+\theta_{2}\right),
$$

where $\theta_{1}$ and $\theta_{2}$ are the polar angles of the vector sum of the track momenta in each hemisphere (described below) corresponding respectively to the outgoing fermion and anti-fermion. This variable preserves the true angular distribution in the $e^{+} e^{-}$centreof-mass when hard collinear radiation takes place from the initial state.

The observed $e^{+} e^{-} \rightarrow e^{+} e^{-}$cross section has contributions from the s-channel, the $t$-channel, and the interference between them. The correction for the $t$-channel contribution is affected by the inaccuracy of the present generators or formulae used to calculate it. In order to keep this uncertainty on the cross section smaller then $0.5 \%$ the angular range is restricted to $-0.9<\cos \theta^{*}<0.7$ and only data at centre-of-mass energies within $1 \mathrm{GeV}$ of the peak are used. Over this angular range the cross sections for the t-channel and the interference have been computed, multiplied by the experimental luminosity and subtracted from the number of efficiency-corrected events. After this subtraction only the s-channel remains, and, for the leptonic width, it can be treated in the same manner as the other lepton channels.

In the selections it is required that all the parts of the detector essential for this analysis are functioning and that all essential triggers are enabled. The following requirements are designed to separate leptonic from hadronic decays and are common to all channels:

1. A track must have at least 4 space points measured in the $\mathrm{TPC}$, have momentum exceeding $0.1 \mathrm{GeV} / \mathrm{c}$, and originate from the beam-crossing within $5 \mathrm{~cm}$ along the beam direction and $1.5 \mathrm{~cm}$ in the transverse direction. 
2. The event is required to have more than one and less than seven tracks in the polar angle range $|\cos \theta|<0.95$.

3. At least one track must have a momentum larger than $3 \mathrm{GeV} / \mathrm{c}$.

4. The event, divided into two hemispheres by a plane perpendicular to the thrust axis, has to have at least one track in each hemisphere.

5. The track momenta in each hemisphere are summed vectorially. The acollinearity $\eta$, defined as $180^{\circ}$ minus the angle between these vector sums, has to be smaller than $20^{\circ}$.

6. Events with more than 4 tracks are rejected if any track makes an angle greater than $31.8^{\circ}$ with the vector sum of the track momenta in the same hemisphere.

The vertex constraint (1)eliminates most tracks which originate from beam-gas interactions, cosmic rays, or are badly measured. The multiplicity cut (2), and the collimation cut (6) remove hadronic $Z$ decays while retaining nearly all the leptonic decays. Two photon interactions are removed by cuts (4) and (5).

To determine the geometrical acceptances and the selection efficiencies, Monte Carlo programs have been used in which the complete detector response is simulated and the events are reconstructed with the same analysis chain as the data.

Since at least two TPC tracks are required, the loss of events due to tracking inefficiencies which may not be included in the Monte Carlo simulation has been investigated. Clean $e^{+} e^{-}$events have been selected from a sub-sample of the data on the basis of ECAL clusters and ITC tracks and the probability that TPC tracks are found pointing to both ECAL clusters is measured as a function of the polar angle.

The main loss of events is at low angles when a track points into a crack between two TPC sectors. A sub-sample of events has been used to investigate this loss. In the range $0.85<|\cos \theta|<0.95$ there are 682 events, of which 62 are near cracks, and in 33 of these events one or both tracks are lost. The Monte Carlo predicts $32 \pm 5$ events lost near cracks, in good agreement with the data. From this we conclude that the cracks are correctly simulated in the Monte Carlo.

In the range $|\cos \theta|<0.9$ and excluding tracks near cracks, 1137 events are found, of which 14 have lost tracks. These 14 events have been visually scanned and 12 are due to hard bremsstrahlung in material before the TPC, causing the reconstructed track to fail the vertex constraint. The other two are due to TPC inefficiency. The Monte Carlo predicts $5.9 \pm 1.7$ events with tracks lost due to bremsstrahlung and none lost due to TPC inefficiency.

Thus for $\mu^{+} \mu^{-}$or $\tau^{+} \tau^{-}$events in the range $|\cos \theta|<0.9$ there is a loss of $(0.18 \pm 0.12) \%$ events due to TPC inefficiencies which are not simulated by the Monte Carlo. For $e^{+} e^{-}$ events, there is a further loss of $(0.54 \pm 0.34) \%$ because there is more hard bremsstrahlung seen in the data than is simulated by the Monte Carlo.

\subsection{The $\mathrm{Z} \rightarrow e^{+} e^{-}$Cross Section}

The selection of $Z \rightarrow e^{+} e^{-}$events from the sample chosen with the above cuts requires tracks with high momenta and large energy deposition in ECAL. Events are selected in 
Figure 11: ECAL energy associated to the two tracks with highest momentum plotted against the sum of the momenta of the two tracks for preselected lepton pair candidates. The dotted line shows the selection cuts for $e^{+} e^{-}$ pairs.

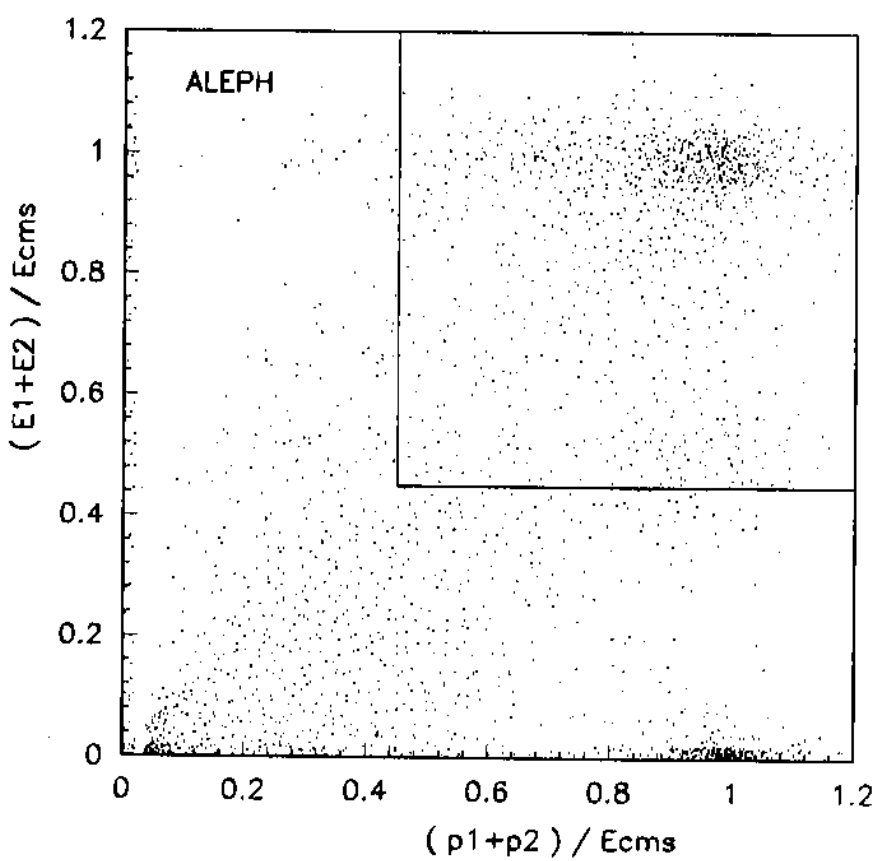

the angular range $-0.9<\cos \theta^{*}<0.7$. They are retained if the sum of the momenta of the two most energetic tracks, and the sum of the cluster energies in the ECAL spatially associated with these tracks both exceed $45 \%$ of the centre-of-mass energy. In Fig. 11 the correlation between the total ECAL energy and the total track momentum is shown for a sub-sample of the preselected events. Below the cuts, indicated as dashed lines, the $\mu$ and $\tau$-pair background events are dominant.

For events in which both selected particles have the same charge (which occurs in only $0.7 \%$ of events for $\left|\cos \theta^{*}\right|<0.9$ and largely arises from the conversion of a hard radiated photon) the particle with the highest momentum is used to select the sign unless the measurement error is very large, in which case the other one is chosen.

These selection criteria are checked by relaxing the requirement of two TPC tracks and demanding only one TPC track opposite an ECAL cluster. The methods differ in only $3 \%$ of the sample, and cross sections calculated using the two selections are consistent to better than $1 \%$. The data are analysed separately for each of the centreof-mass energies used and the number of observed events in the angular range $-0.9<$ $\cos \theta^{*}<0.7$ for each energy is shown in Table 4 .

The selection efficiency has been computed by means of a Monte Carlo simulation using the event generator BABAMC [4]. Although BABAMC is limited to $O(\alpha)$ radiative corrections (no generator is available for $\mathrm{O}\left(\alpha^{2}\right)$ ), it is satisfactory for the purpose of calculating efficiencies. The efficiency has been computed only for those events for which the generated acollinearity is less than $20^{\circ}$. The efficiency is very high $(\sim 99 \%)$ in the central region with a slight but significant fall in the forward and backward regions, caused by loss of energy in insensitive regions between calorimeter modules. The angular distribution at each centre-of-mass energy is corrected by this efficiency, the systematic error of which has been estimated to be less than $1 \%$.

The trigger efficiency has been investigated as described in section 3 and there is no evidence of any inefficiency over the angular region used. Figure 12 shows the corrected angular distribution together with the predicted t-channel contribution. 
Table 4: Event numbers, integrated luminosities, cross sections and forward-backward asymmetries as a function of the centre-of-mass energy for $Z \rightarrow e^{+} e^{-}$. The overall systematic error on the cross sections of $2 \%$ is not included.

\begin{tabular}{|c|c|c|c|c|c|c|}
\hline$\sqrt{s}$ & $\mathcal{L}^{\text {int }}$ & $\begin{array}{c}N_{e^{+} e^{-}} \\
{[-0.9,0.7}\end{array}$ & $\begin{array}{c}N_{\mathrm{t}-\text { chan. }} \\
{[-0.9,0.7]}\end{array}$ & $\begin{array}{c}N_{e^{+} e^{-}} \\
\text {(corrected) }\end{array}$ & $\sigma_{e e}$ & \multicolumn{1}{|c|}{$A_{\mathrm{FB}}$} \\
$(\mathrm{GeV})$ & $\left(\mathrm{nb}^{-1}\right)$ & & & & $(\mathrm{nb})$ & \\
\hline 88.218 & $118.0 \pm 2.0$ & 52 & 25.2 & $36.8 \pm 9.6$ & $0.31 \pm 0.08$ & $-0.04 \pm 0.31$ \\
88.278 & $108.8 \pm 2.0$ & 49 & 23.6 & $35.1 \pm 9.7$ & $0.32 \pm 0.09$ & $-0.30 \pm 0.39$ \\
89.222 & $100.0 \pm 1.9$ & 52 & 23.7 & $38.9 \pm 9.6$ & $0.39 \pm 0.10$ & $-0.27 \pm 0.35$ \\
89.284 & $62.8 \pm 1.5$ & 33 & 15.0 & $24.9 \pm 8.0$ & $0.40 \pm 0.13$ & $-1.43 \pm 1.02$ \\
90.220 & $81.6 \pm 1.7$ & 76 & 20.3 & $76.7 \pm 11.7$ & $0.94 \pm 0.14$ & $-0.35 \pm 0.20$ \\
90.282 & $122.2 \pm 2.1$ & 129 & 30.1 & $136.4 \pm 16.0$ & $1.12 \pm 0.13$ & $-0.18 \pm 0.14$ \\
91.034 & $181.7 \pm 2.6$ & 221 & 29.6 & $265.1 \pm 21.1$ & $1.46 \pm 0.12$ & $0.05 \pm 0.08$ \\
91.220 & $828.2 \pm 5.6$ & 1072 & 110.4 & $1330.2 \pm 44.7$ & $1.61 \pm 0.05$ & $-0.02 \pm 0.04$ \\
91.283 & $209.7 \pm 2.8$ & 226 & 24.1 & $279.2 \pm 21.3$ & $1.33 \pm 0.10$ & $0.04 \pm 0.08$ \\
91.530 & $165.5 \pm 2.5$ & 185 & 12.1 & $240.7 \pm 19.5$ & $1.45 \pm 0.12$ & $-0.06 \pm 0.09$ \\
92.219 & $49.5 \pm 1.4$ & 47 & 0.4 & $65.0 \pm 9.5$ & $1.31 \pm 0.19$ & $0.19 \pm 0.15$ \\
92.280 & $132.2 \pm 2.2$ & 103 & 0.3 & $143.9 \pm 14.6$ & $1.09 \pm 0.11$ & $0.02 \pm 0.11$ \\
93.219 & $139.1 \pm 2.3$ & 70 & 0.0 & $99.2 \pm 11.7$ & $0.71 \pm 0.08$ & $0.31 \pm 0.10$ \\
93.287 & $84.1 \pm 1.8$ & 43 & -0.1 & $61.3 \pm 9.6$ & $0.73 \pm 0.12$ & $0.15 \pm 0.15$ \\
94.214 & $54.7 \pm 1.5$ & 23 & 0.7 & $32.2 \pm 6.8$ & $0.59 \pm 0.13$ & $0.34 \pm 0.19$ \\
94.278 & $75.1 \pm 1.7$ & 23 & 0.7 & $32.0 \pm 7.1$ & $0.43 \pm 0.09$ & $0.29 \pm 0.22$ \\
\hline
\end{tabular}

Figure 12: Distribution of the polar angle for $\mathrm{Z} \rightarrow e^{+} e^{-}$before $t$-channel subtraction. The dashed histogram shows the fit result and the solid one shows the t-channel contribution.

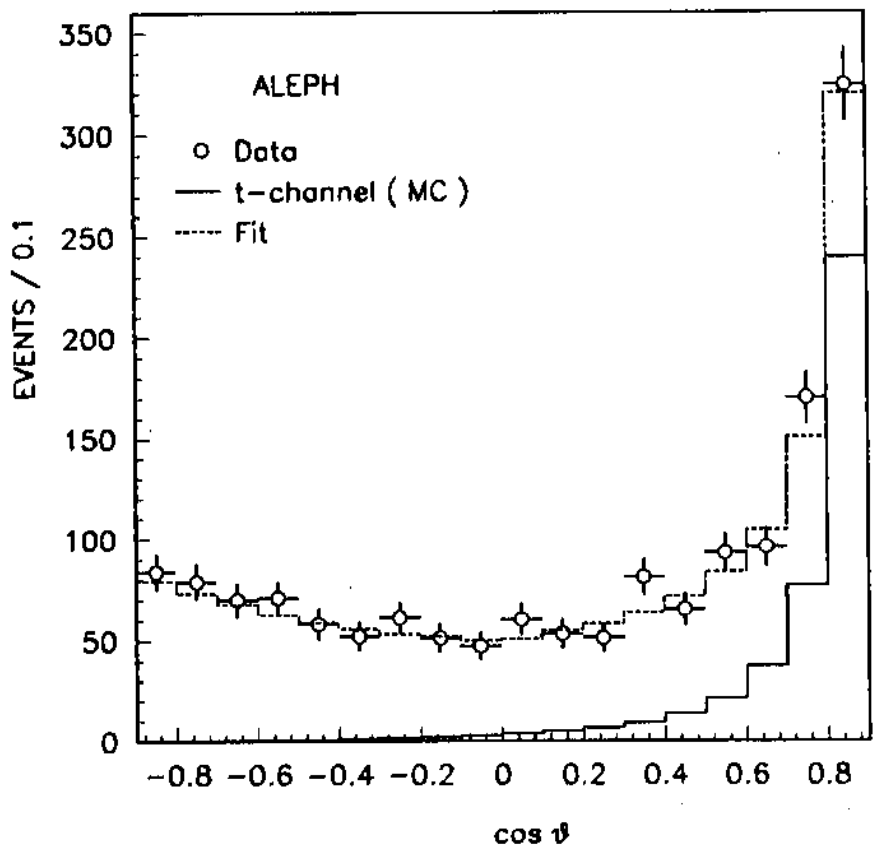


In order to calculate the $t$-channel contribution, since no generator exists which handles $\mathrm{O}\left(\alpha^{2}\right)$ radiative corrections, the cross section has been computed using the formulae of Greco [10] as implemented in a program by Caffo and Remiddi [11]. This program computes the cross sections separately for s-channel, $t$-channel exchange and their interference within certain defined kinematical boundaries and can be used either with $O(\alpha)$ correction, or with multiple soft-photon emission described by exponentiation. When using the latter, the major effects of higher order corrections are included and at the $\mathrm{Z}$ peak, this changes the $\mathrm{t}$-channel contribution by about $15 \%$. The cross section is calculated by the program within cuts. An acollinearity cut of $20^{\circ}$ has been used, as in the data. For the other cut, corresponding to the integration of the radiation from the outgoing particle within a cone, the cone angle is set at $5^{\circ}$. The result is, howewer, not sensitive to the precise value; the difference between cuts of $5^{\circ}$ and $10^{\circ}$ is only $0.1 \%$ in the cross section. Since the analytical formula is obtained by making some approximations in the integration procedure, its validity has been checked by comparing the evaluation of t-channel effects using the formula in first-order approximation with results to the same order from the generators BABAMC and MUONMC [4]. The conclusion is that an error of $3 \%$ should be ascribed to the t-channel subtraction, which includes the normalization error of $1.3 \%$ from the luminosity measurement. Thus in the region $-0.9<\cos \theta^{*}<0.7$, which is the region used for analysis, and where the integrated t-channel contribution is $11 \%$ at the $Z$ peak, the overall systematic uncertainty from t-channel subtraction is about $0.5 \%$ at the five centre-of-mass energies around the peak. Uncertainties in the s-t interference term from the mass and width of the $Z$ are very small.

The only significant background process contaminating the $e^{+} e^{-}$channel is $\tau^{+} \tau^{-}$ decay, while the contributions from $\mu$ pairs, hadrons, and two-photon interactions are negligible. $\tau$-pair events, generated with KORALZ [12], are analysed with the same program chain and the same cuts and analysis procedure. The fraction passing the cuts is $(2.9 \pm 0.2) \%$, uniform in $\cos \theta^{*}$ and this background is subtracted from the t-channel corrected distribution.

Finally, a correction is made for events lost by the $20^{\circ}$ acollinearity cut and by the acceptance cut $-0.9<\cos \theta^{*}<0.7$. This is made using KORALZ since the $s-$ and $\mathrm{t}$-channels have quite different distributions in acollinearity. The correction factor is 1.380 at the $Z$ peak with a statistical error of 0.006 .

The cross section for the pure s-channel part of $e^{+} e^{-} \rightarrow e^{+} e^{-}$is then computed by dividing the angular distributions, corrected for the above effects, by the corresponding integrated luminosity. In Table 4 the number of events observed in the angular range $-0.9<\cos \theta^{*}<0.7$, the number of events computed as the $t$-channel subtraction, and the corrected number of events in the full angular range $\left|\cos \theta^{*}\right|<1.0$, together with the resulting cross sections are listed. No correction for the s-channel one-photon exchange has been applied, and only statistical errors are quoted. The overall systematic error in the cross section of $2 \%$ ( $1.3 \%$ from luminosity, $0.5 \%$ from $t$-channel subtraction, and $1 \%$ from event selection and $\tau$ subtraction) is not included.

The forward-backward asymmetry is determined by a fit of Eq. (1) to the $\cos \theta^{*}$ angular distributions at each centre-of-mass energy using a maximum likelihood method. The results are given in Table 4. 
Figure 13: Distribution of the number of fired planes in the $\mathrm{HCAL}$ relative to the geometrically predicted number; a) for all planes; b) for the last 10 planes. Electrons do not penetrate into the HCAL and are concentrated at zero. Open circles are data and the histogram shows the simulation.

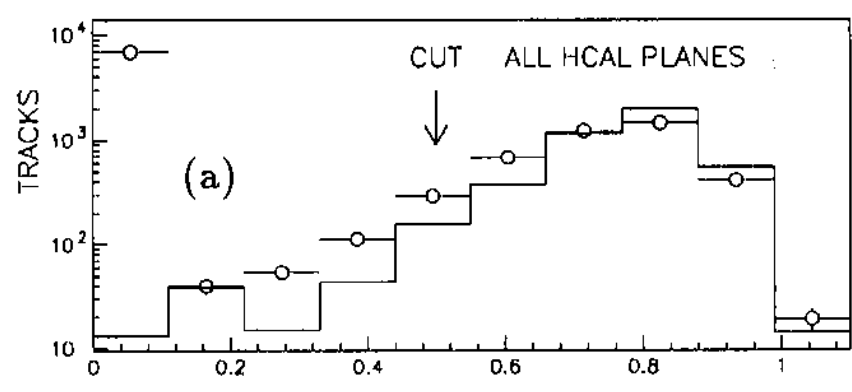

FRACTION OF PLANES

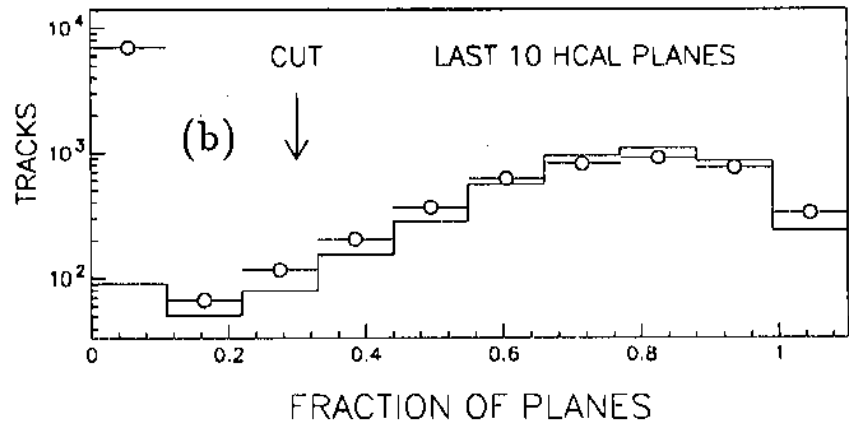

\subsection{The $\mathbf{Z} \rightarrow \mu^{+} \mu^{-}$Cross Section}

The $\mu$-pair events are selected in two steps. First events with at least two high momentum tracks are chosen and then the muons are identified. This requires the proper operation of the HCAL, which slightly reduces the integrated luminosity with respect to the other data samples.

In a preselection, events passing the cuts described in Section 6 are retained for tracks with $|\cos \theta|<0.9$ but with an acollinearity cut enlarged to $\eta<23^{\circ}$. The acceptance after these cuts is $(85.47 \pm 0.35) \%$. The highest momentum is required to be greater than $35 \mathrm{GeV} / \mathrm{c}$ and the second highest has to be greater than $22 \mathrm{GeV} / \mathrm{c}$. In this sample $(97.9 \pm 0.2) \%$ of the $\mu^{+} \mu^{-}$pairs, $<1 \%$ of the $\tau^{+} \tau^{-}$pairs, and the majority of the $e^{+} e^{-}$ pairs are retained. Events with moderate final- or initial-state radiation are included even if one or two photons have created an $e^{+} e^{-}$pair in the material between the interaction point and the TPC. Hadronic $Z$ decays and background from two-photon interactions are negligible after this preselection.

The $\mu^{+} \mu^{-}$pairs are then separated from the $e^{+} e^{-}$pairs, using the digital wire readout of the HCAL, requiring that at least one track penetrates into the iron of the HCAL and that at least one track satisfies the penetration trigger. Each track is extrapolated through the entire apparatus, taking into account the energy loss and multiple scattering in the material as well as the bending due to the magnetic field. The total number of HCAL wire planes which fire inside a $20 \mathrm{~cm}$ wide road around each track must be larger than 6 and larger than $50 \%$ of the number of geometrically expected planes after taking into account the dead zones ( $\sim 5 \%$ by construction and $\sim 2 \%$ from missing electronics). Additionally, in the 10 outermost planes the ratio of the number of fired planes to the expected number has to be larger than $30 \%$. Figure 13 shows these ratios for the preselected events. The $\mu^{+} \mu^{-}$identification probability is measured to be $0.973 \pm 0.001$ by comparing the number of events that have both sides identified with the number of 
Table 5: Event numbers, integrated luminosities, cross sections and forward-backward asymmetries for $\mathrm{Z} \rightarrow \mu^{+} \mu^{-}$decays. The overall systematic error on the cross sections of $1.6 \%$ is not included.

\begin{tabular}{|c|r|r|c|c|}
\hline $\begin{array}{c}\sqrt{s} \\
(\mathrm{GeV})\end{array}$ & \multicolumn{1}{|c|}{$\begin{array}{c}\mathcal{L}^{\text {int }} \\
\left(\mathrm{nb}^{-1}\right)\end{array}$} & $N_{\mu \mu}$ & $\begin{array}{c}\sigma_{\mu \mu} \\
(\mathrm{nb})\end{array}$ & $A_{\mathrm{FB}}$ \\
\hline 88.227 & $118.0 \pm 2.0$ & 28 & $0.28 \pm 0.05$ & $-0.14 \pm 0.20$ \\
88.278 & $108.5 \pm 2.0$ & 20 & $0.22 \pm 0.05$ & $-0.34 \pm 0.20$ \\
89.225 & $100.0 \pm 1.9$ & 49 & $0.59 \pm 0.08$ & $-0.29 \pm 0.11$ \\
89.284 & $44.1 \pm 1.3$ & 11 & $0.30 \pm 0.09$ & $-0.30 \pm 0.20$ \\
90.225 & $81.6 \pm 1.7$ & 64 & $0.94 \pm 0.12$ & $-0.22 \pm 0.11$ \\
90.282 & $70.7 \pm 1.6$ & 54 & $0.93 \pm 0.13$ & $-0.05 \pm 0.10$ \\
91.034 & $147.6 \pm 2.4$ & 159 & $1.30 \pm 0.10$ & $0.02 \pm 0.07$ \\
91.224 & $784.2 \pm 5.2$ & 962 & $1.47 \pm 0.05$ & $-0.01 \pm 0.03$ \\
91.283 & $143.1 \pm 2.3$ & 180 & $1.50 \pm 0.11$ & $-0.03 \pm 0.08$ \\
91.530 & $146.2 \pm 2.4$ & 180 & $1.48 \pm 0.11$ & $0.03 \pm 0.07$ \\
92.221 & $49.5 \pm 1.4$ & 45 & $1.09 \pm 0.16$ & $0.11 \pm 0.10$ \\
92.280 & $114.8 \pm 2.1$ & 101 & $1.05 \pm 0.11$ & $0.14 \pm 0.10$ \\
93.222 & $139.1 \pm 2.3$ & 55 & $0.47 \pm 0.06$ & $0.03 \pm 0.12$ \\
93.287 & $41.7 \pm 1.3$ & 25 & $0.73 \pm 0.14$ & $0.23 \pm 0.17$ \\
94.219 & $54.7 \pm 1.5$ & 24 & $0.52 \pm 0.11$ & $0.11 \pm 0.21$ \\
94.278 & $59.4 \pm 1.6$ & 16 & $0.33 \pm 0.08$ & $0.04 \pm 0.28$ \\
\hline
\end{tabular}

Figure 14: Distribution of the polar angle for $\mathrm{Z} \rightarrow \mu^{+} \mu^{-}$.

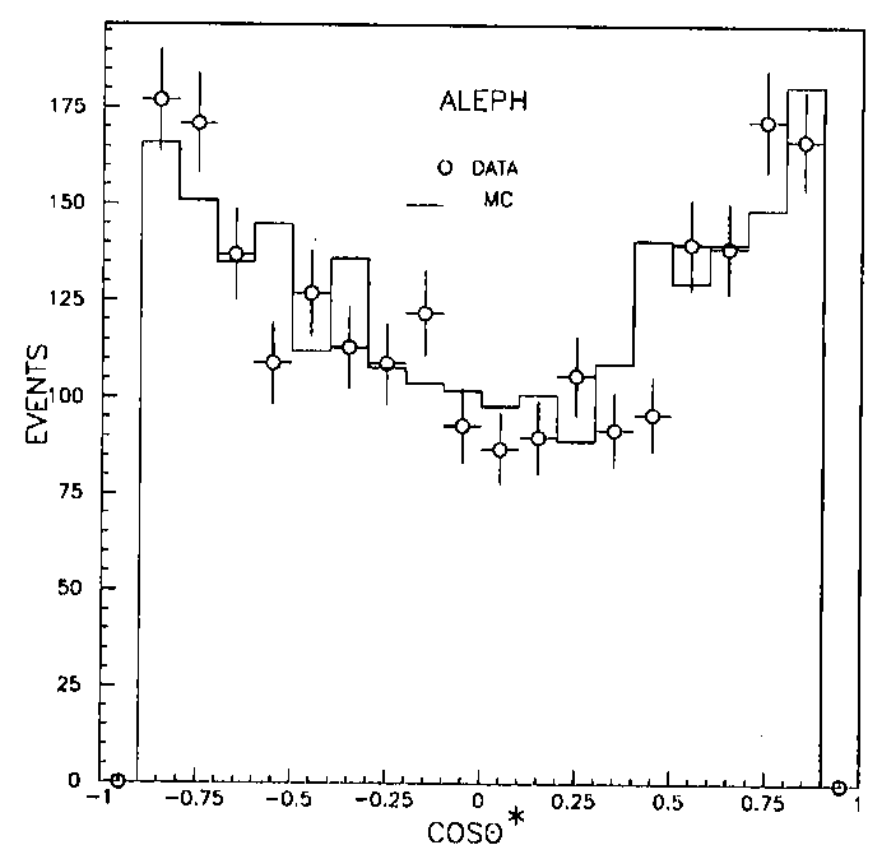


events with only one side identified. The electron-pair contamination is negligible and the $\tau^{+} \tau^{-}$pair contamination is found to be $0.003 \pm 0.003$ from Monte Carlo simulation. Cosmic-ray events are rejected both by cuts on the distance of closest approach of the tracks to the collision point, $4 \mathrm{~cm}$ along the beam and $0.5 \mathrm{~cm}$ transverse, and also the requirement of the HCAL track trigger due to the small time overlap between the ITC and HCAL readouts. The cosmic-ray contamination is less than $10^{-3}$. After these cuts 1973 events remain.

The trigger efficiency has been measured by comparing the number of events in which both muons have triggered with the number of events in which only one muon has triggered and the other is in an active region of the HCAL. The trigger efficiency over the solid angle employed is found to be consistent with $100 \%$.

In an alternative approach $\mu$ pairs are identified on the basis of the energy deposition in the electromagnetic and hadronic calorimeters. Several combinations of ECAL and HCAL energies have been tried yielding basically the same result. The $\tau^{+} \tau^{--}$and $e^{+} e^{-}$ pair background is best suppressed by demanding the maximum energy sum of the ECAL and HCAL to be less than $25 \mathrm{GeV}$ and the minimum energy sum to be less than $12 \mathrm{GeV}$. Comparing the results of these selections with the one based on the HCAL digital readout allows an estimate of the systematic uncertainty in the selection procedure. The agreement is at the level of $0.8 \%$. Combining this result with the error on the trigger efficiency and that from the $\tau^{+} \tau^{-}$background subtraction a total systematic error of $0.9 \%$ is obtained.

In Table 5 cross sections and integrated luminosities are presented for the different centre-of-mass energies. These results are obtained combining the various methods used to select the muon pairs. The errors are statistical only; the systematic errors of $1.3 \%$ from the luminosity and $0.9 \%$ from the selection methods have to be added. The angular distribution of the $\mu^{+} \mu^{-}$events from all the centre-of-mass energies is shown in Fig. 14. The forward-backward asymmetry is determined at each centre-of-mass energy by a maximum likelihood fit of the function given in Eq. (1) to the data. The results are listed in Table 5.

\subsection{The $\mathrm{Z} \rightarrow \tau^{+} \tau^{-}$Cross Section}

The $\tau$-pair events are isolated by applying the following section criteria in addition to the cuts described in Section 6. In each hemisphere we define a "jet" by clustering together all the tracks present in the hemisphere. The direction of the $\tau$ is defined by the vectorial sum of the momenta of all the tracks of the jet (the jet axis). The acollinearity cut is relaxed to include angles up to $26^{\circ}$, while the cosine of the angle between each track and the jet axis is required to be larger than 0.95 . Cosmic-ray events are rejected by demanding that in all events with only two charged tracks the transverse distance to the beam position of at least one of these tracks be less than $5 \mathrm{~mm}$.

A Monte Carlo study has shown that the efficiency of this preselection for $\tau$ pairs is $94 \%$, and that the preselected sample consists of $46.8 \%$ Bhabha, $26.5 \% \mu$, and $24.6 \%$ $\tau$ pairs with $2.0 \%$ of the events coming from background two-photon events and $0.1 \%$ arising from hadronic events.

After the preselection, the process $\mathrm{Z} \rightarrow \tau^{+} \tau^{-}$must be separated from the other lepton species. The dominant background originates from $e$ pairs and a large part of it is 
Figure 15: Total ECAL wire energy relative to the beam energy for the preselected lepton sample compared with Monte Carlo predictions

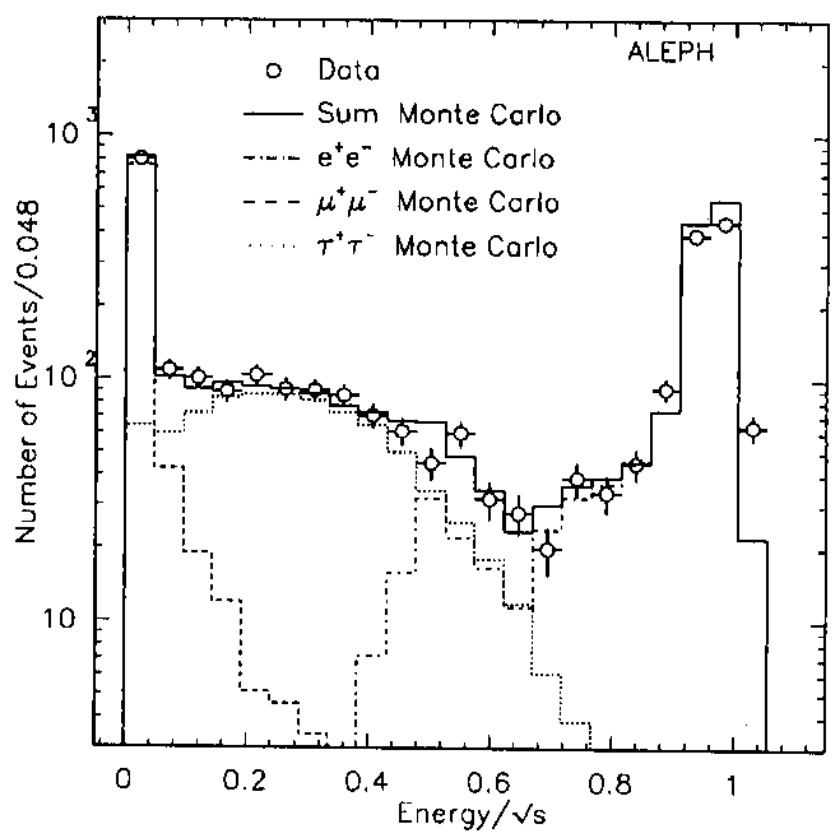

rejected by demanding that the total energy measured by the ECAL (see Fig. 15) be less than $60 \%$ of the centre-of-mass energy. The remainder are rejected with only one further criterion, the square of the missing mass calculated from the detected charged particles (assumed to be massless) and the known centre-of-mass energy. This is required to exceed $400 \mathrm{GeV}^{2}$ (see Fig. 16). This cut also rejects $e$ and $\mu$ pairs when they are produced with an associated photon.

To further reduce the background from two-photon processes it is required that one track in the event has a momentum larger than $5 \mathrm{GeV} / \mathrm{c}$.

Within the range $|\cos \theta|<0.92169$ events are retained with a selection efficeincy of $(86.5 \pm 0.4) \%$. The angular acceptance of this selection for $\mathrm{Z} \rightarrow \tau^{+} \tau^{-}$is $85.7 \%$.

The background from electron pairs has been evaluated by studying the missing mass spectrum of events with ECAL energy larger then $70 \mathrm{GeV}$; it amounts to $(1.2 \pm 0.5) \%$ for the full data sample. The background from muon pair production is negligible and that from cosmic ray muons has been evaluated from the distribution of the transverse distance to the beam position to be $(0.6 \pm 0.4) \%$. Monte Carlo simulations of two-photon and hadronic events have been compared with the data and have been used to estimate the contamination to be $(3.3 \pm 1.4) \%$. The overall background subtraction amounts to $(5.1 \pm 1.4) \%$.

The main uncertainty in the acceptance is due to the error in the momentum scale of the TPC; a smaller error is due to the uncertainty in the energy scale of the ECAL. The overall effect is evaluated to be $1 \%$. The trigger efficiency has been measured using the selected sample by comparing the number of events with one or two triggers and a correction of $0.997 \pm 0.001$ has been applied. Figure 17 shows the scalar sum of the momenta of the charged particles and Fig. 18 shows the angular distribution of the selected sample compared with the Monte Carlo prediction.

An alternative approach for $\tau$-pair selection[1] is based on the identification and rejection of $\mu^{+} \mu^{-} \gamma$ and $e^{+} e^{-} \gamma$ events from the preselected sample, using the measured 
Figure 16: Missing mass squared for the full lepton sample with ECAL wire energy $<0.6 \sqrt{s}$. It is compared with Monte Carlo predictions.

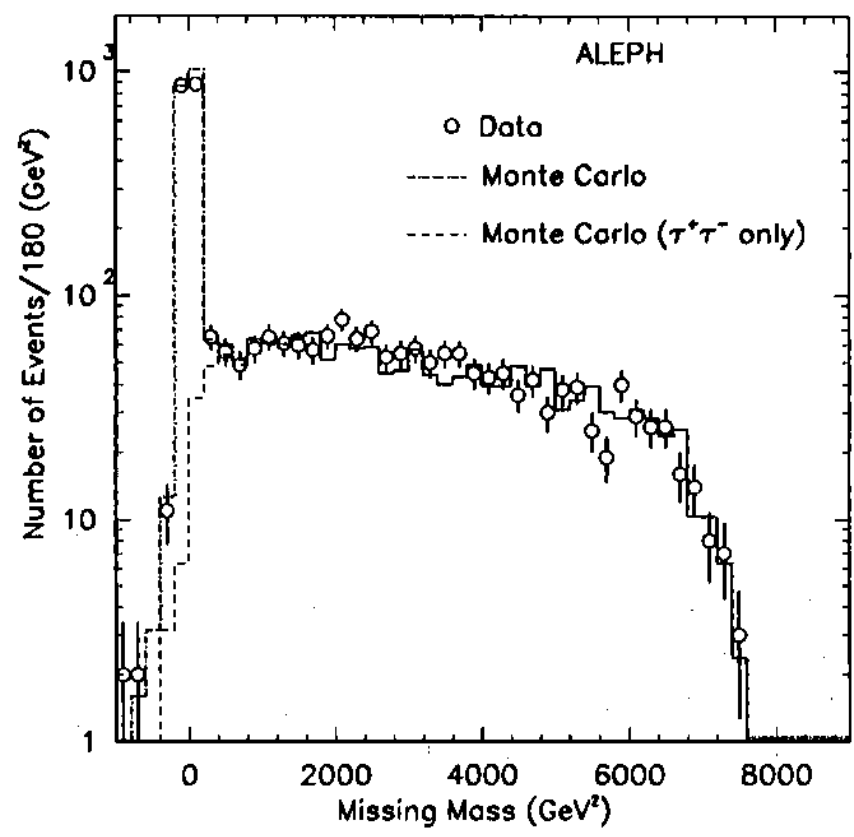

momenta in the TPC, the cluster energies detected in the ECAL, and the patterns from the digital wire readout of the HCAL. This procedure accepts events in the angular range $|\cos \theta|<0.95$. The cross section computed with this method has been compared with the selection already described and an agreement at the level of $1.4 \%$ has been found which is compatible with the statistical fluctuation due to events not in common $(\sim 20 \%$ of the total sample). This comparison has allowed a reduction of the systematic error due to the background subtraction.

Table 6 shows the average of the cross sections evaluated from the two selections with the statistical error taking into account the correlations due to the common sample of events. The overall scale errors due to luminosity (1.3\%) and selection procedure (1\%) are not included. The error due to the background subtraction which has been applied to average cross sections is also not included in the table. The background contamination is estimated differently for the two selection procedures and the two errors are almost independent. The resulting error on the average cross section due to background subtraction is estimated to be $9 \mathrm{pb}$.

In the $\tau^{+} \tau^{-}$decay the asymmetry measurement depends on the contamination from the $e^{+} e^{-}$pairs because of the strong forward asymmetry of the $t$-channel. The event selection used for the cross section measurement is insufficient and has to be extended to reject remaining $e^{+} e^{-}$pairs by identifying either a $\pi$ or $\mu$ in the final state. For each charged track with momentum greater than $3 \mathrm{GeV} / \mathrm{c}$ the energy deposited in the first stack of the ECAL is compared with the expected value for a minimum ionizing particle. If the probability is larger than $5 \%$ for either track, the event is selected.

The $\tau$ direction and charge are defined as the sum of momenta and the sum of the charges of the charged particles in its decay jet. Using KORALZ, the difference between the real and the reconstructed $\tau$ direction, one finds a dispersion of $30 \mathrm{mrad}$, is negligible for the asymmetry measurement.

Events are studied in the angular region $\left|\cos \theta^{*}\right|<0.9$, where $\theta^{*}$ is defined in Eq. (2). In order to have a clear charge assignment, only events with opposite charge sums are 
Figure 17: Scalar sum of the momenta of the charged particles in the selected $\tau$-pair sample. The solid line is the Monte Carlo prediction.
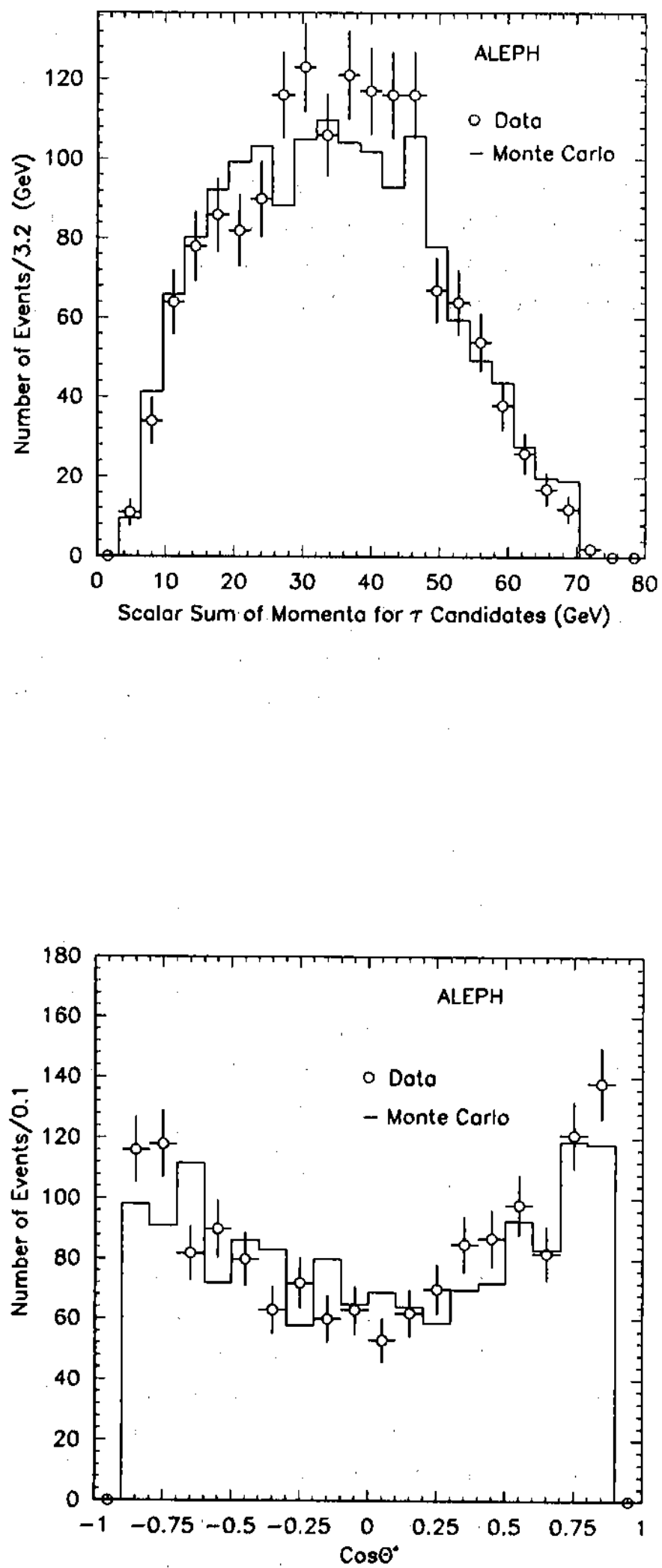
Table 6: Event numbers, integrated luminosities, cross sections, and forward-backward asymmetries for $\mathrm{Z} \rightarrow \tau^{+} \tau^{-}$decays. The systematic errors of $1.7 \%$ and $9 \mathrm{pb}$ in the cross sections are not included.

\begin{tabular}{|c|r|r|r|r|}
\hline $\begin{array}{c}\sqrt{s} \\
(\mathrm{GeV})\end{array}$ & $\begin{array}{r}\mathcal{L}^{\text {int }} \\
\left(\mathrm{nb}^{-1}\right)\end{array}$ & $N_{\tau \tau}$ & $\begin{array}{c}\sigma_{\tau \tau} \\
(\mathrm{nb})\end{array}$ & \multicolumn{1}{c|}{$A_{\mathrm{FB}}$} \\
\hline 88.227 & $118.0 \pm 2.1$ & 16 & $0.14 \pm 0.05$ & $-0.02 \pm 0.12$ \\
88.278 & $108.8 \pm 2.0$ & 31 & $0.31 \pm 0.06$ & $-0.70 \pm 0.18$ \\
89.225 & $99.98 \pm 1.9$ & 30 & $0.36 \pm 0.07$ & $0.07 \pm 0.24$ \\
89.284 & $62.8 \pm 1.5$ & 26 & $0.46 \pm 0.09$ & $-0.17 \pm 0.21$ \\
90.225 & $73.9 \pm 1.7$ & 54 & $0.93 \pm 0.14$ & $0.01 \pm 0.12$ \\
90.282 & $122.2 \pm 2.1$ & 92 & $0.91 \pm 0.10$ & $0.00 \pm 0.12$ \\
91.034 & $181.7 \pm 2.6$ & 202 & $1.38 \pm 0.10$ & $-0.04 \pm 0.08$ \\
91.224 & $828.2 \pm 5.6$ & 985 & $1.52 \pm 0.05$ & $0.04 \pm 0.04$ \\
91.283 & $209.7 \pm 2.8$ & 233 & $1.46 \pm 0.09$ & $0.05 \pm 0.08$ \\
91.530 & $165.5 \pm 2.5$ & 195 & $1.45 \pm 0.10$ & $-0.12 \pm 0.08$ \\
92.221 & $49.5 \pm 1.1$ & 40 & $1.03 \pm 0.17$ & $-0.24 \pm 0.21$ \\
92.280 & $132.3 \pm 2.2$ & 115 & $1.05 \pm 0.10$ & $0.27 \pm 0.09$ \\
93.222 & $139.1 \pm 2.3$ & 66 & $0.59 \pm 0.08$ & $-0.10 \pm 0.14$ \\
93.287 & $84.1 \pm 1.8$ & 46 & $0.67 \pm 0.10$ & $0.32 \pm 0.16$ \\
94.219 & $54.7 \pm 1.5$ & 15 & $0.32 \pm 0.10$ & $-0.07 \pm 0.20$ \\
94.278 & $75.1 \pm 1.7$ & 23 & $0.33 \pm 0.08$ & $-0.21 \pm 0.23$ \\
\hline
\end{tabular}

retained. At each energy the values of $\cos \theta^{*}$ are entered in a likelihood function which is maximized over the range $\left.\mid \cos \theta^{*}\right\}<0.9$. The corrected asymmetry over the whole range $\left|\cos \theta^{*}\right|<1$ is computed from Eq. (1).

The systematic uncertainty resulting from t-channel $e^{+} e^{-}$contamination is below $2 \%$ at all centre-of-mass energies and below $1 \%$ at the $\mathrm{Z}$ peak. Since it is small with respect to the statistical error it is neglected.

In Table 6 the forward-backward asymmetry is listed for the different centre-of-mass energies.

\subsection{The $\mathrm{Z} \rightarrow l^{+} l^{-}$Cross Section}

A selection of $\mathrm{Z}$ decays into lepton pairs $\left(\mathrm{Z} \rightarrow l^{+} l^{-}\right)$, without distinguishing the final-state flavour, is made using the cuts described in Section 6, but with some extra conditions to reduce the residual background of cosmic rays and two-photon interactions:

1. For two-track events at least one track is required to originate from the beamcrossing within $1 \mathrm{~cm}$ in the transverse direction and $5 \mathrm{~cm}$ in the direction along the beam.

2. The transverse momentum relative to the beam of the vector sum of the tracks in each hemisphere (the jet transverse momentum) must be larger than $2.5 \mathrm{GeV}$ in at least one of the two hemispheres. 
Figure 19: Total track momentum distribution for selected lepton events in the centre-of-mass energy range of 91 to $92 \mathrm{GeV}$.

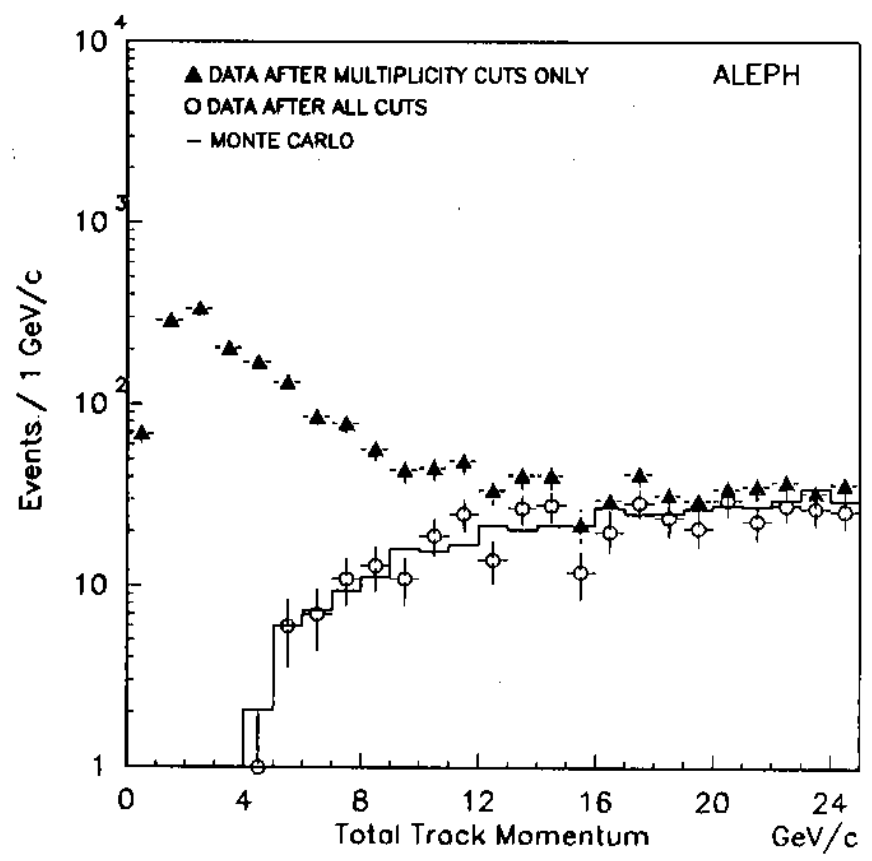

3. For two-track events with both momenta below $6 \mathrm{GeV}$, the ratio of transverse momenta with respect to the beam must differ from unity by more than $15 \%$.

4. The event has to satisfy the condition $-0.90<\cos \theta^{*}<0.70$.

Cut 3 is designed to remove two-photon interactions, which in general have an almost balanced final-state transverse momentum. On the other hand, $\tau$ events, with which they tend to be confused, have in general an unbalanced final-state transverse momentum, especially in the case of low visible momenta when the neutrinos take most of the energy. The last cut is applied to reduce the systematic error due to t-channel subtraction. These cuts result in an efficient selection of leptonic $Z$ decays with little background from hadronic $\mathrm{Z}$ decays, cosmic rays or two-photon interactions. Figure 19 shows the visible momentum spectrum for the events selected by charged multiplicity only (triangles) and by all cuts (open points). The selection cuts affect mainly the region of small visible momentum where the excess of events correspond mainly to twophoton interactions. It has been verified that the energy dependence of the cross section for these events is non-resonant. The solid histogram in Fig. 19 shows the momentum spectrum with all cuts applied for Monte Carlo simulated leptonic Z decays and is in good agreement with the data.

The angular distribution of the $l^{+} l^{-}$events is shown in Fig. 20 together with the expectation from Monte Carlo simulation.

The total background after cuts is estimated from Monte Carlo for hadronic and two-photon events and from data for cosmic-rays. The total background is $(1.3 \pm 0.2) \%$; it is evaluated as a function of $\cos \theta^{*}$ and subtracted bin by bin.

The selection efficiency is estimated by Monte Carlo simulation. The $e^{+} e^{-}$events are generated with the BABAMC generator, whilst $\mu^{+} \mu^{-}$and $\tau^{+} \tau^{-}$events are generated with KORALZ. The resulting combined efficiency in the region of acollinearity $\eta$ less than $20^{\circ}$ is $98.7 \%$ (99.7\% for $e^{+} e^{-}, 99.9 \%$ for $\mu^{+} \mu^{-}$, and $96.4 \%$ for $\tau^{+} \tau^{-}$events). 
Table 7: Event numbers, integrated luminosities, cross sections, and forward-backward asymmetries for $Z \rightarrow$ lepton pairs as a function of the centre-of-mass energy. The overall systematic error of $1.5 \%$ for $\sqrt{s}$ between $89.22 \mathrm{GeV}$ and $93.28 \mathrm{GeV}$ is not included. For points outside this range the systematic error increases and amounts to $1.7 \%$ at $\sqrt{s}=88.227 \mathrm{GeV}$.

\begin{tabular}{|c|r|r|r|r|c|c|}
\hline$\sqrt{s}$ & $\mathcal{L}^{\text {int }}$ & $\begin{array}{r}N_{\text {leptons }} \\
{[-0.9,0.7]}\end{array}$ & $\begin{array}{r}N_{\text {t }- \text { chan. }}[-0.9,0.7] \\
(\mathrm{GeV})\end{array}$ & \multicolumn{1}{c|}{$\begin{array}{c}N_{\text {leptons }}^{\text {corr }} \\
{[-1,1]}\end{array}$} & $\sigma_{l^{+} l^{-}}$ & $A_{\mathrm{FB}}$ \\
\hline 88.227 & $118.0 \pm 2.0$ & 90 & 25.2 & $87.2 \pm 13.0$ & $0.74 \pm 0.11$ & $-0.19 \pm 0.19$ \\
88.278 & $108.8 \pm 2.0$ & 99 & 23.6 & $99.7 \pm 13.7$ & $0.92 \pm 0.13$ & $-0.45 \pm 0.20$ \\
89.225 & $100.0 \pm 1.9$ & 122 & 23.7 & $132.8 \pm 15.1$ & $1.33 \pm 0.15$ & $-0.27 \pm 0.16$ \\
89.284 & $62.8 \pm 1.5$ & 69 & 15.0 & $71.7 \pm 11.4$ & $1.14 \pm 0.18$ & $-0.61 \pm 0.23$ \\
90.225 & $81.6 \pm 1.7$ & 191 & 20.3 & $234.2 \pm 19.2$ & $2.87 \pm 0.24$ & $-0.07 \pm 0.09$ \\
90.282 & $122.2 \pm 2.1$ & 299 & 30.1 & $369.0 \pm 24.1$ & $3.02 \pm 0.20$ & $-0.04 \pm 0.07$ \\
91.034 & $181.7 \pm 2.6$ & 580 & 29.6 & $761.9 \pm 34.0$ & $4.19 \pm 0.20$ & $+0.04 \pm 0.05$ \\
91.224 & $828.3 \pm 5.6$ & 2853 & 110.4 & $3804.9 \pm 75.9$ & $4.59 \pm 0.10$ & $+0.01 \pm 0.02$ \\
91.283 & $209.7 \pm 2.8$ & 673 & 24.1 & $899.0 \pm 36.7$ & $4.29 \pm 0.18$ & $+0.01 \pm 0.04$ \\
91.530 & $165.5 \pm 2.5$ & 533 & 12.1 & $726.7 \pm 32.8$ & $4.39 \pm 0.21$ & $-0.04 \pm 0.05$ \\
92.221 & $49.5 \pm 1.4$ & 122 & 0.4 & $171.1 \pm 15.7$ & $3.46 \pm 0.33$ & $+0.16 \pm 0.10$ \\
92.280 & $132.2 \pm 2.2$ & 283 & 0.3 & $396.8 \pm 24.1$ & $3.00 \pm 0.19$ & $+0.10 \pm 0.06$ \\
93.222 & $139.1 \pm 2.4$ & 199 & 0.0 & $282.8 \pm 20.4$ & $2.03 \pm 0.15$ & $+0.18 \pm 0.08$ \\
93.287 & $84.1 \pm 1.8$ & 124 & -0.1 & $175.9 \pm 16.1$ & $2.09 \pm 0.20$ & $+0.17 \pm 0.10$ \\
94.219 & $54.7 \pm 1.5$ & 55 & 0.7 & $78.6 \pm 11.0$ & $1.44 \pm 0.20$ & $+0.15 \pm 0.14$ \\
94.278 & $75.1 \pm 1.7$ & 67 & 0.7 & $94.1 \pm 12.1$ & $1.25 \pm 0.16$ & $+0.10 \pm 0.13$ \\
\hline
\end{tabular}

Figure 20: Angular distribution of lepton-pair events in the centre-of-mass energy range of 91 to $92 \mathrm{GeV}$. The solid line is the Monte Carlo prediction.

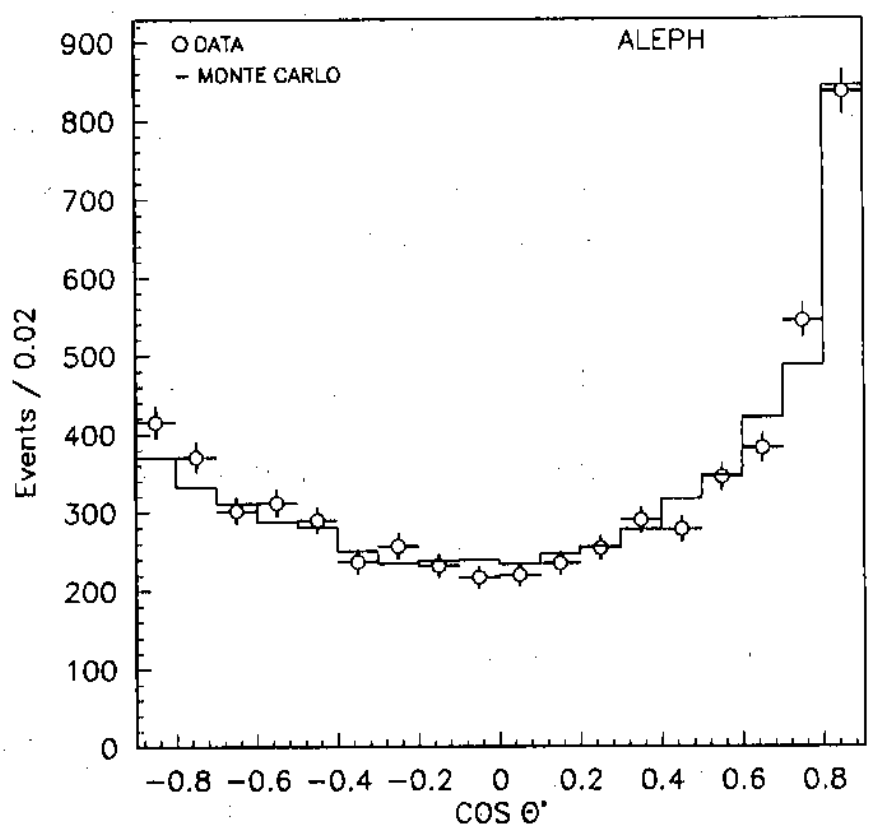


The systematic error on the selection efficiency is mainly due to the uncertainty of the momentum scale of the TPC and to tracking inefficiencies and has been estimated to be $0.5 \%$. The trigger efficiency is measured by comparing the number of events in which only one or both leptons have triggered. The resulting efficiency is $(99.9 \pm 0.1) \%$.

After background subtraction and correction for selection and trigger efficiencies the contribution from t-channel photon exchange, which contributes to the Bhabha events, is subtracted from the data using the same method described in Section 6.1. For the calculation of asymmetries the t-channel contribution is evaluated as a function of $\cos \theta^{*}$ and subtracted bin by bin for each energy. The correction becomes large at small $\cos \theta^{*}$, but the overall subtraction is relatively less important than for Bhabha scattering alone, since the sample includes also $\mu$ and $\tau$ decays of the $\mathrm{Z}$. It contributes $0.2 \%$ to the systematic error in the cross section for the points with $\left|\sqrt{s}-M_{z}\right|<1.5 \mathrm{GeV}$ which are those used in the fits. The systematic error becomes larger outside this range.

Once the $t$-channel contribution is subtracted the event sample is corrected for the loss resulting from the acollinearity cut as well as for acceptance. Both are computed with the program KORALZ for each energy. At the peak of the $\mathrm{Z}$ resonance the combined acollinearity and angular acceptance is $(72.2 \pm 0.2) \%$ ( $73.2 \%$ angular acceptance and $98.5 \%$ acollinearity acceptance). Far from the peak the loss of events due to the acollinearity cut increases and is as large as $5 \%$ at $\sqrt{s}=88.28 \mathrm{GeV}$.

Table 7 shows the cross sections with statistical exrors only at different centre-of-mass energies. The overall systematic error for the cross sections in the range $\left|\sqrt{s}-M_{Z}\right|<$ $1.5 \mathrm{GeV}$ is $1.5 \%$ ( $1.3 \%$ from luminosity , $0.2 \%$ from t-channel subtraction and $0.6 \%$ from event selection).

The forward-backward asymmetry is calculated at each energy by fitting the $\cos \theta^{*}$ distribution, after the t-channel subtraction, according to Eq. (1) using a $\chi^{2}$ minimization method. The resulting coefficients $A_{\mathrm{FB}}$ are shown in Table 7.

\section{The Z Resonance Parameters}

The cross section $\sigma_{e^{+} e^{-} \rightarrow f f}$ for fermion-pair production in $e^{+} e^{-}$annihilation, after correction for initial-state radiation, can be expressed in a model-independent formulation $[13,14,15]$ as a function of the physical parameters of the $\mathrm{Z}$ resonance. It contains three terms:

- The contribution of the $\mathrm{Z}$ exchange that is represented by a Breit-Wigner function.

- The contribution of the photon exchange.

- The interference term.

To a good approximation it is given by:

$$
\begin{aligned}
\sigma(s)_{f f}= & \frac{s}{\left(s-M_{\mathrm{Z}}^{2}\right)^{2}+s^{2} \Gamma_{\mathrm{Z}}^{2} / M_{\mathrm{Z}}^{2}}\left(\frac{12 \pi \Gamma_{f f}}{M_{\mathrm{Z}}^{2}} \frac{\Gamma_{e e}}{1+\frac{3}{4} \frac{\alpha(0)}{\pi}}+I_{f} \frac{N_{\mathrm{c}}\left(s-M_{\mathrm{Z}}^{2}\right)}{s}\right) \\
& +\frac{4}{3} \pi N_{\mathrm{c}} \mathrm{Q}_{f}^{2} \frac{\alpha^{2}(s)}{s}
\end{aligned}
$$


where $\Gamma_{e e}, \Gamma_{f f}$ are the partial widths for $\mathrm{Z}$ decay into $e^{+} e^{-}$or any fermion pair $f \bar{f} ; \mathrm{Q}_{\mathrm{f}}$ is the charge and $N_{c}$ is the colour factor of the fermion. Here, $\Gamma_{\mathrm{Z}}$ is the total width, and $M_{\mathrm{Z}}$ the mass of the $Z$ boson. The invisible width is defined by $\Gamma_{\text {inv }}=\Gamma_{Z}-\Gamma_{\text {had }}-\Gamma_{e e}-\Gamma_{\mu \mu}-\Gamma_{\tau \tau}$ with $\Gamma_{\text {had }}$ denoting the hadronic decay width. In this formula $\Gamma_{e e}$ is divided by the factor $\left(1+\frac{3}{4} \frac{\alpha(0)}{\pi}\right)$ to allow for a separate treatment of the initial state radiation [16], as described below. In a completely model independent description, the magnitude of the interference term, $I_{f}$, is unknown. However, the results depend only weakly on the precise value of $I_{f}$ and so we assume the Standard Model value.

The effect of initial-state radiation must be taken into account when comparing the measurements with the predicted cross sections. This is done by convoluting the expression of the cross section with a suitable radiator function $[15,17]$. The effect is large, of the order of $30 \%$ at the peak, but is known to better than $0.5 \%$ [14] of the cross section.

The peak cross section due to $\mathrm{Z}$ exchange, when unfolded from initial-state radiation, is

$$
\sigma_{f f}^{0}=\frac{12 \pi}{M_{\mathrm{Z}}^{2}} \frac{\Gamma_{e e} \Gamma_{f f}}{\Gamma_{\mathrm{Z}}^{2}}
$$

The resonance parameters have been determined using three different computer programs which calculate the initial-state radiation and the contribution from Z-photon interference terms. The first two programs, based on formulae by Borelli et al. [13] and by Berends et al. [14], use analytic expressions, while the third one following Burgers [15] uses a numerical integration. Differences in the results from the three programs are less than $30 \%$ of the experimental error. The results quoted are from the program using the formula of Burgers [15].

The cross sections are fitted by minimizing $\chi^{2}$ taking into account the various correlations in the data resulting from a common luminosity determination and event selection criteria.

The hadronic cross sections presented in Table 3 and Fig. 21, and leptonic cross sections in Tables $4-7$ and Fig. 22 are used. For the electron-pair sample and the lepton sample without distinction of the final states, points with $\left|\sqrt{s}-M_{\mathrm{Z}}\right|>1.5 \mathrm{GeV}$ are omitted in order to minimize the uncertainty due to the t-channel subtraction far from the peak.

Fitting the hadronic and the three lepton-pair cross sections simultaneously one can determine six parameters: the $\mathrm{Z}$ mass, $M_{\mathrm{Z}}$, the full width, $\Gamma_{\mathrm{Z}}$, the peak hadronic cross section, $\sigma_{\text {had }}^{0}$, and the three ratios of hadron to lepton partial widths, $\Gamma_{\text {had }} / \Gamma_{e e}, \Gamma_{\text {had }} / \Gamma_{\mu \mu}$, $\Gamma_{\text {had }} / \Gamma_{\tau \tau}$. The results are:

$$
\begin{gathered}
M_{\mathrm{Z}}=\left(91.194 \pm 0.016_{\exp } \pm 0.030_{\mathrm{LEP}}\right) \mathrm{GeV} \\
\Gamma_{\mathrm{Z}}=(2489 \pm 30) \mathrm{MeV} \\
\sigma_{\text {had }}^{0}=(41.70 \pm 0.66) \mathrm{nb} \\
\Gamma_{\text {had }} / \Gamma_{e e}=(20.23 \pm 0.61) \\
\Gamma_{\text {had }} / \Gamma_{\mu \mu}=(21.52 \pm 0.55) \\
\Gamma_{\text {had }} / \Gamma_{\tau \tau}=(21.17 \pm 0.54)
\end{gathered}
$$


Figure 21: Cross section for $e^{+} e^{-} \rightarrow$ hadrons as a function of the centre-of-mass energy together with the result of the fit of Eq. (3).

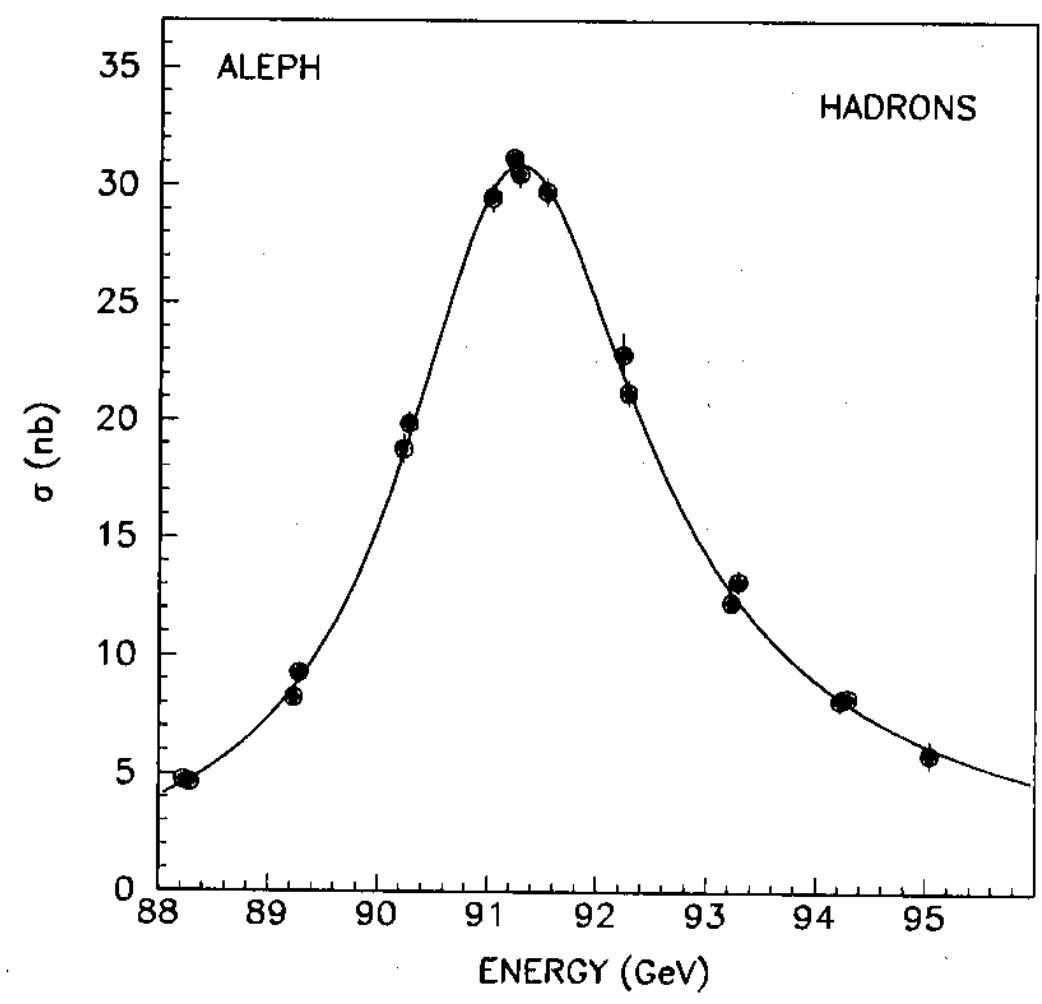

Table 8: $\mathrm{Z}$ resonance parameters derived from the six-parameter fit to the measured hadron and lepton cross sections.

\begin{tabular}{|l|l|c|l|}
\hline Final state & $\begin{array}{l}\text { Partial width } \\
(\mathrm{MeV})\end{array}$ & Branching ratio & $\begin{array}{l}\text { Peak cross section } \\
\text { (nb) }\end{array}$ \\
\hline Hadrons & $1722 \pm 34$ & $0.691 \pm 0.012$ & $41.70 \pm 0.66$ \\
$e^{+} e^{-}$ & $85.0 \pm 1.6$ & $0.0342 \pm 0.0006$ & $2.06 \pm 0.07$ \\
$\mu^{+} \mu^{-}$ & $80.0 \pm 2.5$ & $0.0321 \pm 0.0010$ & $1.94 \pm 0.06$ \\
$\tau^{+} \tau^{-}$ & $81.3 \pm 2.5$ & $0.0331 \pm 0.0010$ & $1.99 \pm 0.06$ \\
Invisible & $521 \pm 34$ & - & - \\
\hline
\end{tabular}

the $\chi^{2}$ is 47 for 52 degrees of freedom. These parameters are generally uncorrelated; the most important correlation is between the hadronic peak cross section and the total width (-28\%).

The second error on the $\mathrm{Z}$ mass is due to the uncertainty in the LEP centre-of-mass energy [18]. The beam energy is stable to a few parts in $10^{-5}$ [18] from one fill to the next.

Other parameters, including the partial widths and branching ratios, can be derived from these results (see Table 8). However, the errors on these parameters have important correlation coefficients, in particular $\Gamma_{e e}$ is strongly anticorrelated with $\Gamma_{\mu \mu}$ and $\Gamma_{\tau \tau}$. The three leptonic widths agree with each other as expected from lepton universality.

Assuming lepton universality we repeat the fit for four parameters, $M_{\mathrm{Z}}, \sigma_{\mathrm{hnd}}^{0}, \Gamma_{\mathrm{Z}}$, $\Gamma_{\ell \ell} / \Gamma_{\text {had }}$ using the hadron sample and the lepton sample selected without distinguishing the final-state flavour (see Fig. 22d). Here, $\Gamma_{\ell \ell}$ is defined as $1 / 3$ of $\Gamma_{\text {leptons. }}$. 
ALEPH
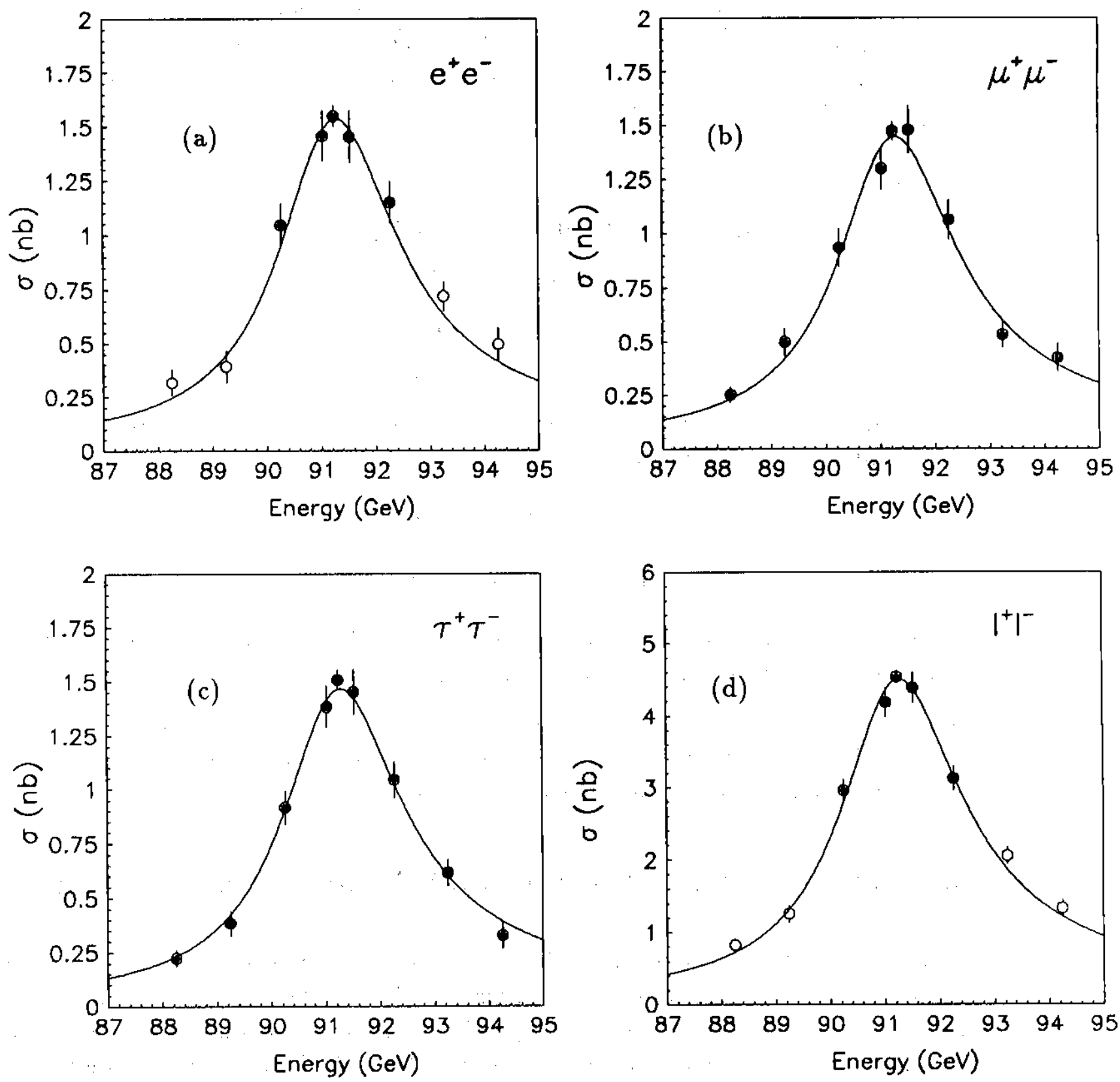

Figure 22: Cross sections for $e^{+} e^{-} \rightarrow$ lepton pairs as a function of the centre-of-mass energy. For points where the energy difference is less than $100 \mathrm{MeV}$ the average cross section is plotted. The full line is result of the fit of Eq. (3). : Points with open circles are not used in the fit. a) $e^{+} e^{-} \rightarrow e^{+} e^{-}$, b) $e^{+} e^{-} \rightarrow \mu^{+} \mu^{-}$, c) $e^{+} e^{-} \rightarrow \tau^{+} \tau^{-}$and d) $e^{+} e^{-} \rightarrow$ lepton pairs. 
Table 9: $\mathrm{Z}$ resonance parameters derived from the four parameter fit to the measured hadron and the combined lepton cross sections.

\begin{tabular}{|l|c|c|c|}
\hline Final state & $\begin{array}{c}\text { Partial width } \\
(\mathrm{MeV})\end{array}$ & Branching ratio & $\begin{array}{c}\text { Peak cross section } \\
(\mathrm{nb})\end{array}$ \\
\hline Hadrons & $1754 \pm 27$ & $0.703 \pm 0.009$ & $41.88 \pm 0.66$ \\
Lepton & $84.3 \pm 1.3$ & $0.0338 \pm 0.0004$ & $2.01 \pm 0.04$ \\
Invisible & $489 \pm 24$ & - & - \\
\hline
\end{tabular}

The result is:

$$
\begin{gathered}
M_{\mathrm{Z}}=\left(91.193 \pm 0.016_{\exp } \pm 0.030_{\mathrm{LEP}}\right) \mathrm{GeV} \\
\Gamma_{\mathrm{Z}}=(2497 \pm 31) \mathrm{MeV} \\
\sigma_{\text {had }}^{0}=(41.86 \pm 0.66) \mathrm{nb} \\
\Gamma_{\text {had }} / \Gamma_{\ell \ell}=20.82 \pm 0.37
\end{gathered}
$$

with $\chi^{2}=18$ for 22 degrees of freedom. Equivalently, the results can be expressed by $\Gamma_{\ell}$ and $\Gamma_{\text {inv }} / \Gamma_{\ell}$ rather than by $\sigma_{\text {had }}^{0}$ and $\Gamma_{Z}$ :

$$
\begin{gathered}
\Gamma_{\ell}=(84.3 \pm 1.3) \mathrm{MeV} \\
\Gamma_{\text {inv }} / \Gamma_{\ell}=5.81 \pm 0.27
\end{gathered}
$$

From these parameters and the correlation between the peak cross section and the full width one obtains the values given in Table 9 .

If one uses the average of the cross sections from the three identified lepton-pair samples instead of the combined lepton sample, one obtains $\Gamma_{\ell}=(83.3 \pm 1.3) \mathrm{MeV}$, which is slightly smaller though statistically consistent.

\section{The Determination of Vector and Axial-vector Couplings}

The measurement of the leptonic width $\Gamma_{\ell}$ permits a determination of the leptonic vector and axial-vector coupling constants or alternatively the electroweak mixing parameter (see sec. 9.2). The high experimental precision, however, requires a consideration of $\mathrm{O}(\alpha)$ electroweak corrections. In Born approximation, the leptonic width is given by

$$
\Gamma_{\ell \ell}=\frac{G_{\mathrm{F}} M_{\mathrm{Z}}^{3}}{6 \sqrt{2} \pi}\left(g_{\mathrm{V} \ell}^{2}+g_{\mathrm{A} \ell}^{2}\right)
$$

In the "improved Born approximation" $[19,20]$, which includes the bulk of the $O(\alpha)$ electroweak corrections, the coupling constants are "running". Their numerical values refer to $\mathrm{Q}^{2}=M_{\mathrm{Z}}^{2}$ and depend on the unknown masses of the top quark and of the Higgs boson.

We adopt here a definition which preserves approximately the established numerical values of the coupling constants and concentrates all the $\mathrm{Q}^{2}$-dependence and the dependence on unknown mass values in the effective running coupling constants $g_{\mathrm{V} \ell}^{2}\left(M_{\mathrm{Z}}^{2}\right)$ and 

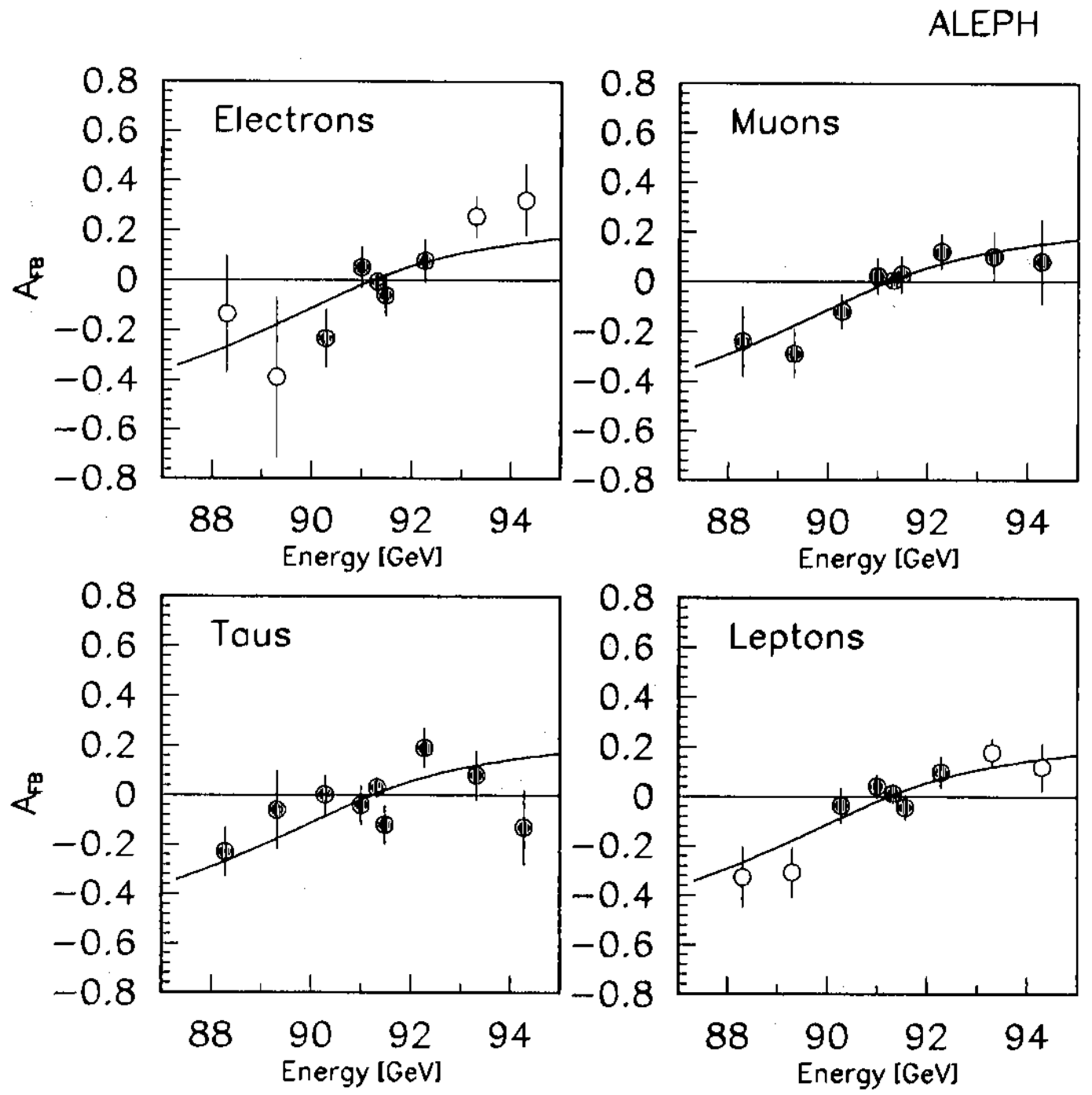

Figure 23: Forward-backward asymmetry for $e^{-}, \mu^{-}, \tau^{-}$, and $l^{-}$in lepton-pair events as a function of the centre-of-mass energy. For points where the energy difference is less than $100 \mathrm{MeV}$ the average asymmetry is plotted. The full lines are the results of the fit. Points with open circles are not used in the fit. 
Figure 24: Probability contours for $g_{\mathrm{V} \ell}\left(M_{\mathrm{Z}}^{2}\right)$ and $g_{\mathrm{A} \ell}\left(M_{\mathrm{Z}}^{2}\right)$.

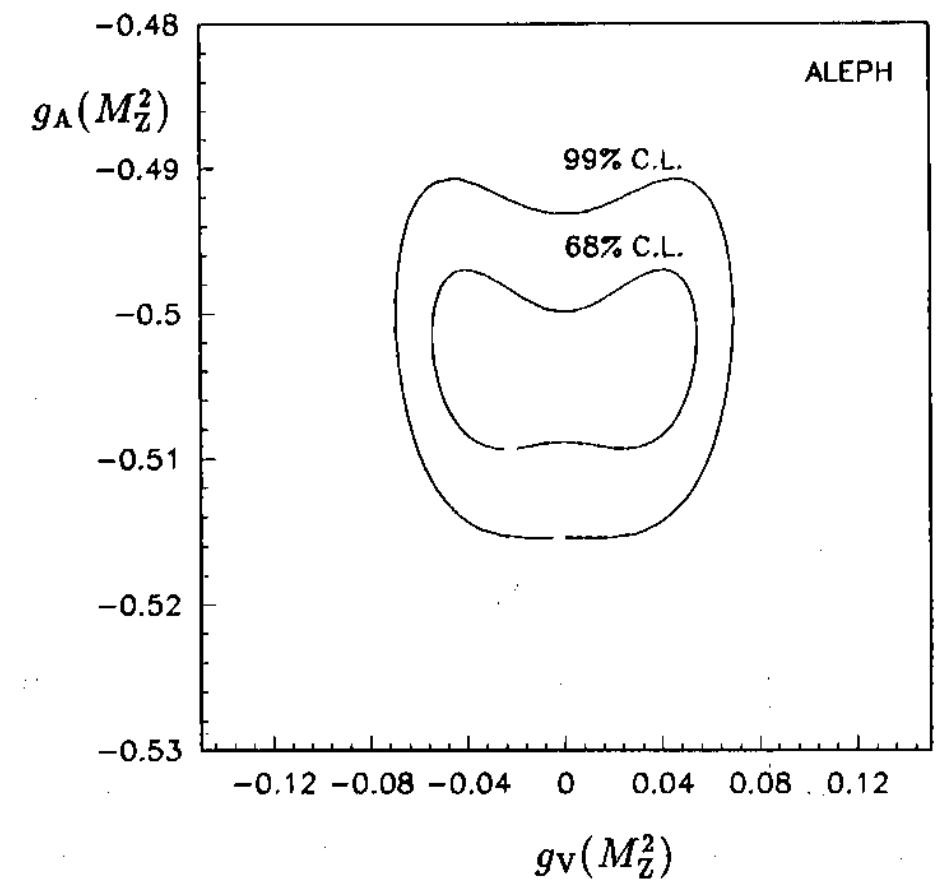

$g_{\mathrm{A} \ell}^{2}\left(M_{\mathrm{Z}}^{2}\right)$. Since they are determined from the measured leptonic width they are valid, in the limit of the improved Born approximation, whatever the mass values of the top quark and the Higgs boson. Defining a dimensionless constant $\mathrm{F}$,

$$
F=\frac{G_{\mathrm{F}} M_{\mathrm{Z}}^{2}}{2 \sqrt{2} \pi \alpha\left(M_{\mathrm{Z}}^{2}\right)}=1.406
$$

we obtain

$$
\Gamma_{\ell \ell}=F \alpha\left(M_{\mathrm{Z}}^{2}\right) \frac{M_{\mathrm{Z}}}{3}\left(g_{\mathrm{V} \ell}^{2}\left(M_{\mathrm{Z}}^{2}\right)+g_{\mathrm{A} \ell}^{2}\left(M_{\mathrm{Z}}^{2}\right)\right)
$$

The forward-backward asymmetry in the angular distribution also depends on $g_{\mathrm{V} \ell}\left(M_{\mathrm{Z}}^{2}\right)$ and $g_{\mathrm{A} \ell}\left(M_{\mathrm{Z}}^{2}\right)$. At the $\mathrm{Z}$ peak the asymmetry is:

$$
A_{\mathrm{FB}}=3 \frac{g_{\mathrm{Ve}}\left(M_{\mathrm{Z}}^{2}\right) g_{\mathrm{Ae}}\left(M_{\mathrm{Z}}^{2}\right)}{g_{\mathrm{Ae}}^{2}\left(M_{\mathrm{Z}}^{2}\right)+g_{\mathrm{Ve}}^{2}\left(M_{\mathrm{Z}}^{2}\right)} \frac{g_{\mathrm{Vf}}\left(M_{\mathrm{Z}}^{2}\right) g_{\mathrm{Af}}\left(M_{\mathrm{Z}}^{2}\right)}{g_{\mathrm{Af}}^{2}\left(M_{\mathrm{Z}}^{2}\right)+g_{\mathrm{Vf}}^{2}\left(M_{\mathrm{Z}}^{2}\right)} .
$$

Off the peak its variation with $s$ depends mainly on $g_{\mathrm{Ae}}\left(M_{\mathrm{Z}}^{2}\right) g_{\mathrm{Af}}\left(M_{\mathrm{Z}}^{2}\right)$.

Different fits have been performed to the measured asymmetries as a function of $\sqrt{s}$ in order to extract the electroweak parameters. Since the statistics are not large, we have assumed lepton universality and the data from e-pair, $\mu$-pair, and $\tau$-pair events have been fit simultaneously. In the $e^{+} e^{-}$channel, only the five points closest to the $\mathrm{Z}$ peak have been used owing to uncertainty in the $t$-channel subtraction at centre-of-mass energies when far from the peak.

Higher order electroweak effects have been taken into account in the fits. Nonphotonic corrections are included by writing the tree-level asymmetry in terms of effective couplings and using the same s-dependence for the $\mathrm{Z}$ width as is used in the line-shape fits. Photonic corrections are added by convoluting the non-radiative forward and backward cross sections with a suitable radiator function. Different theoretical calculations [21], implementing the ideas described above, with different approximations, have been used to fit the data. The results are very similar. 
Figure 25: Contours of constant $\chi^{2}$ for the hadronic peak cross section $\sigma_{\text {had }}^{0}$ as a function of $\Gamma_{\text {had }} / \Gamma_{\ell}$ together with the Standard Model prediction. The uncertainty in the QCD correction is indicated by the error bar and corresponds to $\Delta \alpha_{s}= \pm 0.02$

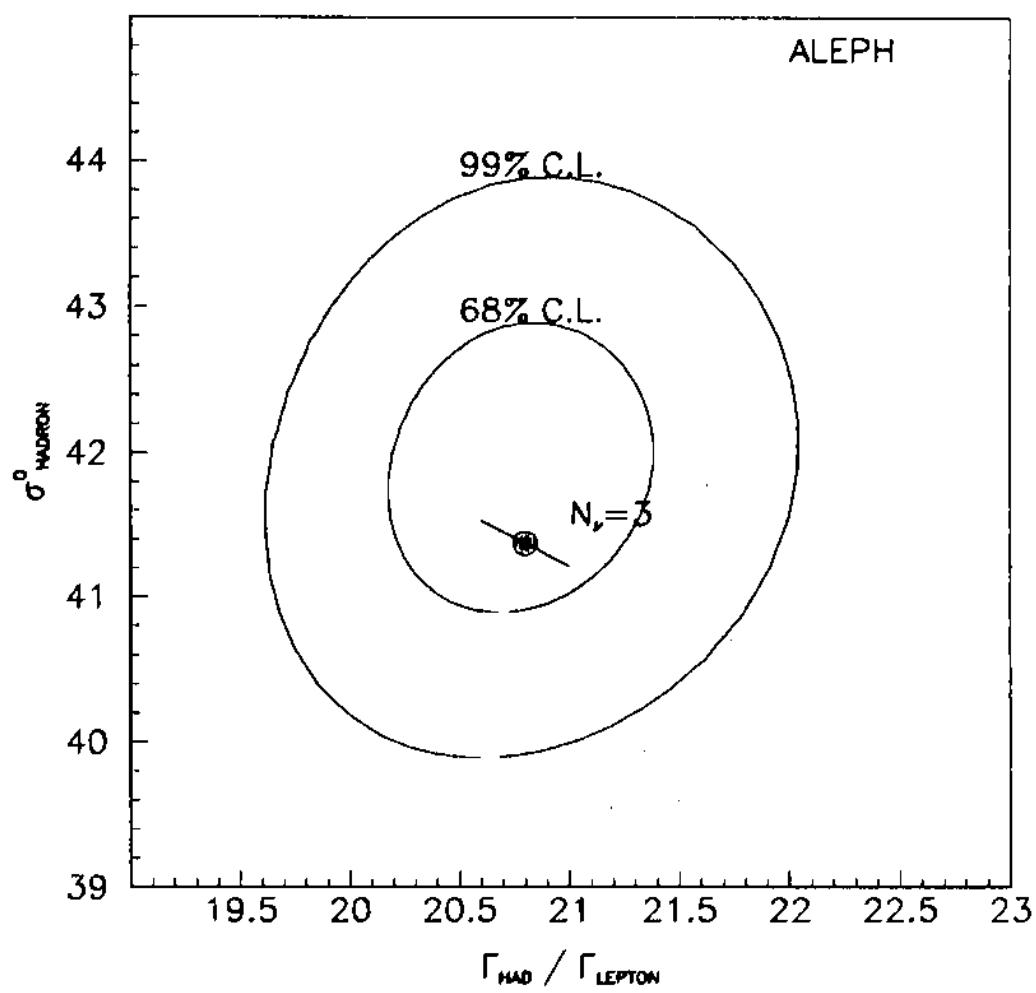

In the fit the values of $M_{\mathrm{Z}}$ and $\Gamma_{\mathrm{Z}}$ from section 7 are used together with the constraint on $g_{\mathrm{V} \ell}^{2}\left(M_{\mathrm{Z}}^{2}\right)$ and $g_{\mathrm{A} \ell}^{2}\left(M_{\mathrm{Z}}^{2}\right)$ from the lepton width, Eq. 5. The latter essentially fixes the value of $g_{\mathrm{A} \ell}^{2}\left(M_{\mathrm{Z}}^{2}\right)$ and its error, since $g_{\mathrm{V} \ell}^{2}\left(M_{\mathrm{Z}}^{2}\right) \ll g_{\mathrm{A} \ell}^{2}\left(M_{\mathrm{Z}}^{2}\right)$. A two parameter fit to the data with the constraint provided by the width yields:

$$
g_{\mathrm{V} \ell}^{2}\left(M_{\mathrm{Z}}^{2}\right)=(0.12 \pm 0.12) \times 10^{-2} \text { and } g_{\mathrm{A} \ell}^{2}\left(\dot{M}_{\mathrm{Z}}^{2}\right)=0.2528 \pm 0.0040
$$

with $\chi^{2}=17$ for 22 degrees of freedom. The errors in the fitted parameters are Gaussian since $g_{\mathrm{A} \ell}^{2}\left(M_{\mathrm{Z}}^{2}\right)$ is essentially proportional to $\Gamma_{\ell \ell}$ and $g_{\mathrm{V} \ell}^{2}\left(M_{\mathrm{Z}}^{2}\right)$ is related linearly to the asymmetry. According to the fit $g_{\mathrm{V} \ell}^{2}\left(M_{\mathrm{Z}}^{2}\right)$ has a non-negligible probability to be in the unphysical region $g_{\mathrm{V} \ell}^{2}\left(M_{\mathrm{Z}}^{2}\right)<0$.

The asymmetries for each lepton species and the fitted curves are shown in Fig. 23.

The data have also been fitted directly with $g_{\mathrm{V} \ell}\left(M_{\mathrm{Z}}^{2}\right)$ and $g_{\mathrm{A} \ell}\left(M_{\mathrm{Z}}^{2}\right)$ which results in a non Gaussian error on $g_{\mathrm{V} \ell}\left(M_{\mathrm{Z}}^{2}\right)$. The probability contours for $g_{\mathrm{A} \ell}\left(M_{\mathrm{Z}}^{2}\right)$ and $g_{\mathrm{V} \ell}\left(M_{\mathrm{Z}}^{2}\right)$ are shown in Fig. 24. When one compares this result with the one obtained in the neutrino-electron scattering experiments $[22,23,24]$ only one solution remains:

$$
g_{\mathrm{V} \ell}\left(M_{\mathrm{Z}}^{2}\right)=-0.034_{-0.014}^{+0.034} \text { and } g_{\mathrm{A} \ell}\left(M_{\mathrm{Z}}^{2}\right)=-0.5029 \pm 0.0041 \text {. }
$$

\section{$9 \quad$ Standard Model Interpretation}

With the new precision for the value of the $\mathrm{Z}$ mass, the Standard Model predictions are substantially better constrained, with only a small dependence on the as yet unknown Higgs and top masses, which enter through higher order corrections. The ratio of partial widths such as $R=\Gamma_{\text {lnd }} / \Gamma_{\ell}$ or the cross sections at the peak dependent very little on $M_{\text {top }}$ and $M_{\text {Higss }}$ and are therefore particularly suitable for a critical comparison with 
Figure 26: Contours of constant $\chi^{2}$ for $\Gamma_{z}$ as a function of $\Gamma_{\text {had }} / \Gamma_{\mu}$ together with the Standard Model predictions for various top masses. The uncertainty due to the QCD correction is indicated by the error bars.

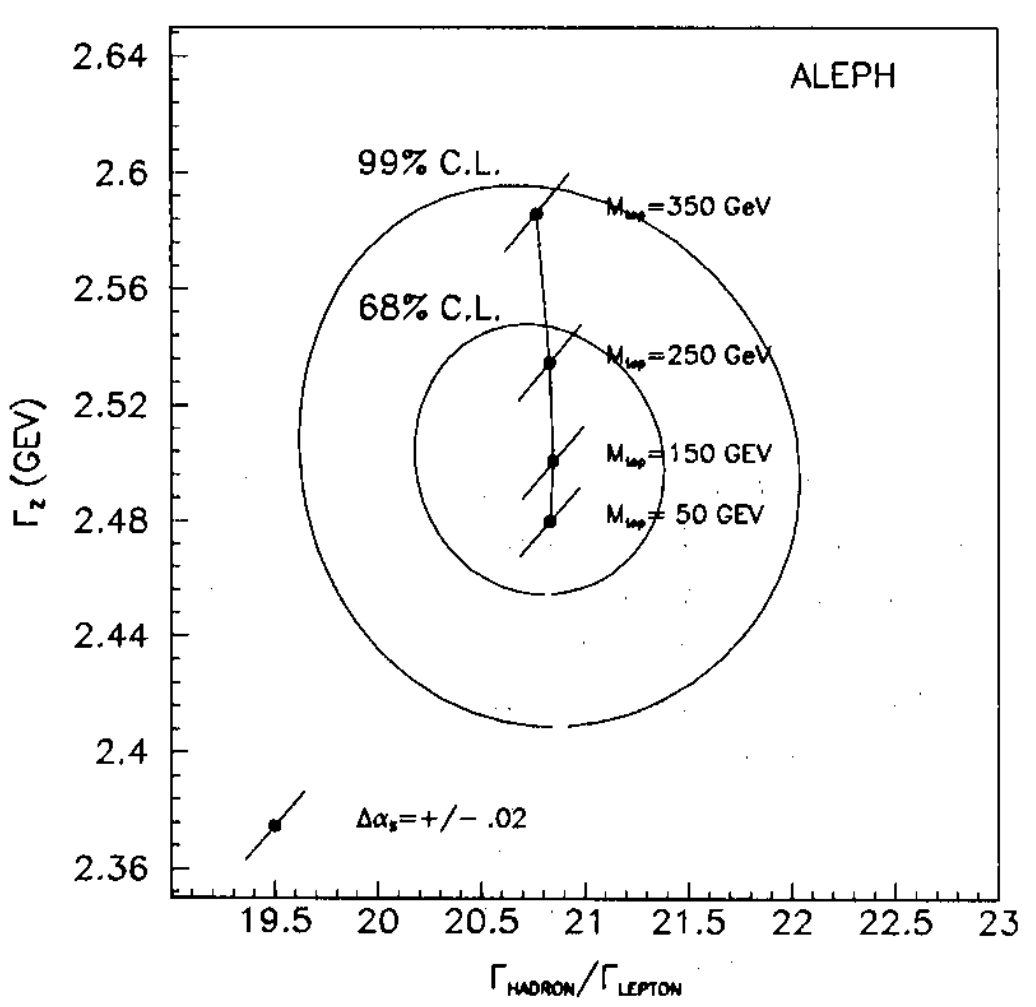

the experiments. The partial widths themselves, are, however, sensitive to $\sin ^{2} \theta_{\mathrm{W}}\left(M_{\mathrm{Z}}^{2}\right)$ and hence to the top mass.

In Fig. 25 the correlation between the peak cross section for $\mathrm{Z}$ decay into hadrons and the ratio of partial widths $R$ is compared with the model prediction for three neutrino species. The main uncertainty in the prediction is due to the uncertainty [25] in the strong interaction correction $\alpha_{\mathrm{s}} / \pi$ and is small compared with the experimental error. The agreement is excellent. Each of the two measurements checks the model with a precision of $\sim 1.5 \%$.

In Fig. 26 the results for $\Gamma_{Z}$ and $R$ are compared with the model. The variation of the theoretical prediction with $M_{\text {top }}$ is indicated as well as the uncertainty due to the $\alpha_{s}$ correction. Again the agreement is excellent for a top mass of the order of $150 \mathrm{GeV}$.

The dependence of $R$ on $M_{\text {top }}$ and $M_{\text {Higgs }}$ can be factorized from the dependence on $\alpha_{s}$. Assuming a first order expansion in $\alpha_{s}$

$$
R=R^{o}\left(1+\frac{\alpha_{s}\left(M_{\mathrm{Z}}^{2}\right)}{\pi}\right)
$$

and using the Standard Model prediction for $R^{\circ}$, the measured value of $R$ yields:

$$
\alpha_{s}=0.13 \pm 0.05_{s t a t} \pm 0.03_{\text {sys }} \text {. }
$$

\subsection{The Measurement of the Number of Light Neutrino Species}

The number of light neutrino species can be obtained from the measured cross sections assuming $\Gamma_{\mathrm{inv}}=N_{\nu} \Gamma_{\nu}$. With

$$
\Gamma_{\mathrm{z}}=\Gamma_{\text {had }}+3 \Gamma_{\ell}+N_{\nu} \Gamma_{\nu}
$$


Figure 27: Cross sections for

$e^{+} e^{-} \rightarrow$ hadrons as a function of the centre-of-mass energy. For points where the energy difference is less than $100 \mathrm{MeV}$ the average cross section is plotted. The Standard Model predictions for $N_{\nu}=2,3$, and 4 are shown.

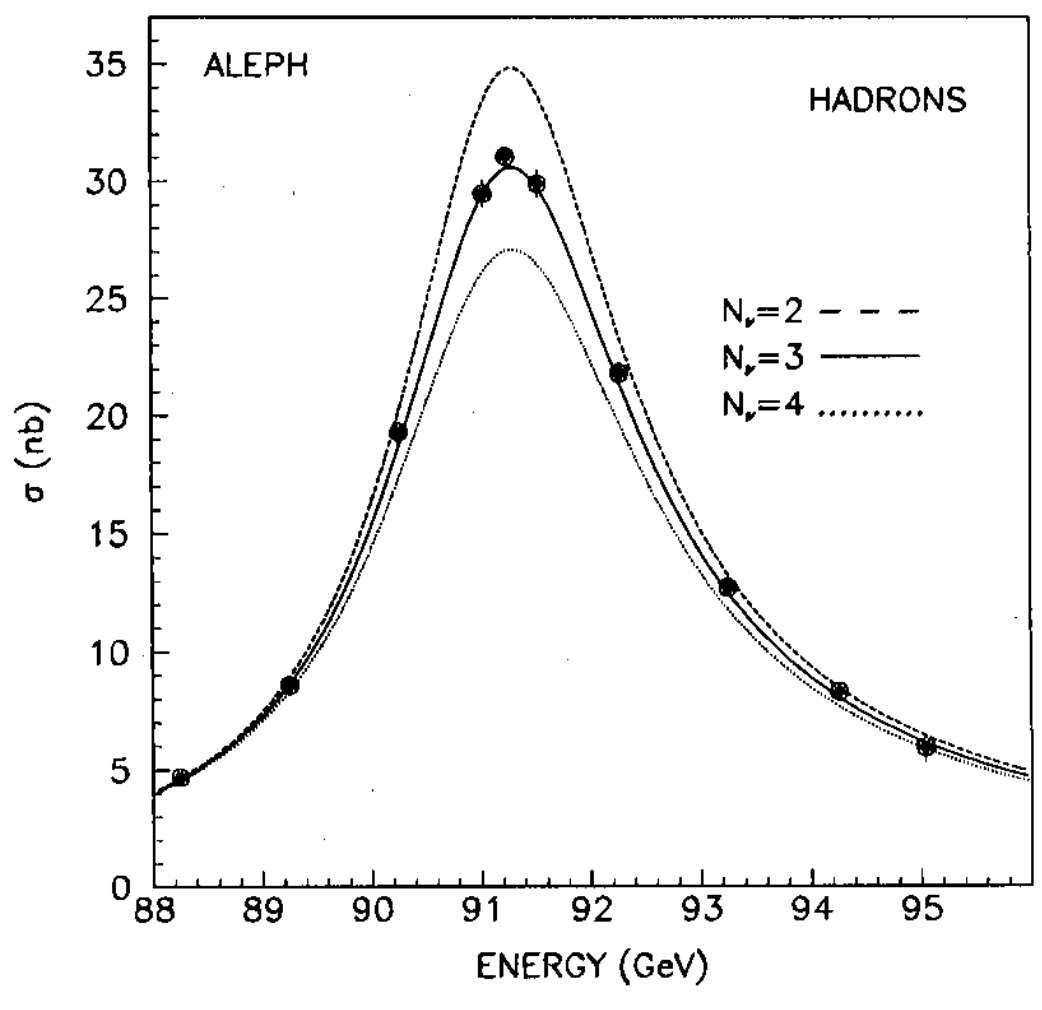

$$
N_{\nu}=\frac{\Gamma_{\mu}}{\Gamma_{\nu}}\left(\sqrt{\frac{12 \pi R}{M_{\mathrm{Z}}^{2} \sigma_{\mathrm{had}}^{0}}}-R-3\right) .
$$

Using the Standard Model prediction for $\Gamma_{U} / \Gamma_{\nu}=0.5010 \pm 0.0005$ and our measured values of $M_{\mathrm{Z}}, \sigma_{\text {had }}^{0}$ and $R$ one finds:

$$
N_{\nu}=2.91 \pm 0.13
$$

The only Standard Model assumption for this result is the ratio $\Gamma_{\ell} / \Gamma_{\nu}$ and hence it is still valid if unexpected states yielding hadrons are present in $\mathrm{Z}$ decay. The expected line shape for hadronic $\mathrm{Z}$ decay together with the data is shown in Fig. 27 for 2,3 , and 4 neutrino species.

\subsection{The Determination of $\sin ^{2} \theta_{\mathrm{W}}\left(M_{\mathrm{Z}}^{2}\right)$}

In the same spirit as in section 8 , one can define ${ }^{2}$ a running electroweak mixing parameter $\sin ^{2} \theta_{\mathrm{W}}\left(M_{\mathrm{Z}}^{2}\right)$ such that with equation 5 and the relation

$$
M_{\mathrm{Z}}^{2}=\frac{\pi \alpha\left(M_{\mathrm{Z}}^{2}\right)}{\sqrt{2} G_{F}(1+\Delta \rho) \sin ^{2} \theta_{\mathrm{W}}\left(M_{\mathrm{Z}}^{2}\right) \cos ^{2} \theta_{\mathrm{W}}\left(M_{\mathrm{Z}}^{2}\right)},
$$

\footnotetext{
${ }^{2}$ The sine of the effective weak mixing angle is defined as the ratio of the running QED and weak coupling constants evaluated at the $Z$ mass:

$$
\sin ^{2} \theta_{\mathrm{W}}\left(M_{\mathrm{Z}}^{2}\right)=\frac{\mathrm{e}^{2}\left(M_{\mathrm{Z}}^{2}\right)}{g^{2}\left(M_{\mathrm{Z}}^{2}\right)} \approx 1-\frac{M_{\mathrm{W}}^{2}}{M_{\mathrm{Z}}^{2}} \frac{1}{(1+\Delta \rho)}
$$

It is defined in different ways in the literature: $\sin ^{2} \hat{\theta}_{\mathrm{W}}\left(M_{\mathrm{Z}}^{2}\right) \overline{M S}[26], \sin ^{2} \theta_{\mathrm{W}}^{*}\left(M_{\mathrm{Z}}^{2}\right)[20]$, $\sin ^{2} \bar{\theta}_{\mathrm{W}}\left(M_{\mathrm{Z}}^{2}\right)[19]$ but the numerical differences are insignificant at the current level of precision.
} 
valid in the improved Born approximation, and expressing

$$
\begin{gathered}
g_{\mathrm{V} \ell}^{2}\left(M_{\mathrm{Z}}^{2}\right)=(1 / 2)^{2}(1+\Delta \rho)(1+\kappa)\left(1 / 2-2 \sin ^{2} \theta_{\mathrm{W}}\left(M_{\mathrm{Z}}^{2}\right)\right)^{2} \text { and } \\
g_{\mathrm{A} \ell}^{2}\left(M_{\mathrm{Z}}^{2}\right)=(1 / 2)^{2}(1+\Delta \rho)(1+\kappa),
\end{gathered}
$$

one obtains

$$
\Gamma_{\ell \ell}=(1+\kappa) \frac{\alpha\left(M_{\mathrm{Z}}^{2}\right) M_{\mathrm{Z}}}{12 \sin ^{2} \theta_{\mathrm{W}}\left(M_{\mathrm{Z}}^{2}\right) \cos ^{2} \theta_{\mathrm{W}}\left(M_{\mathrm{Z}}^{2}\right)} \quad\left[\left(1 / 2-2 \sin ^{2} \theta_{\mathrm{W}}\left(M_{\mathrm{Z}}^{2}\right)\right)^{2}+(1 / 2)^{2}\right]
$$

where $\kappa=(0.2 \pm 0.3) 10^{-2}$ forces the $\mathrm{Z}$ line shape to correspond to an s-dependent Breit. Wigner shape at the $O(\alpha)$ level $[20,27]$ and the dependence on unknown mass parameters are concentrated in $\sin ^{2} \theta_{\mathrm{W}}\left(M_{\mathrm{Z}}^{2}\right)$.

From our measurement of $\Gamma_{\ell \ell}$ and $M_{Z}$ one finds:

$$
\sin ^{2} \theta_{\mathrm{W}}\left(M_{\mathrm{Z}}^{2}\right)=0.2291 \pm 0.0040
$$

which in the limit of the improved Born approximation is valid irrespective of the masses of the top quark and the Higgs boson and is even independent of the weak isospin structure of the Higgs sector of the theory.

The forward-backward asymmetry $A_{\mathrm{FB}}$, discussed in Section 8 furnishes a direct measurement of the ratio of the vector to the axial-vector couplings, $g_{\mathrm{V} \ell}^{2}\left(M_{\mathrm{Z}}^{2}\right) / g_{\mathrm{A} \ell}^{2}\left(M_{\mathrm{Z}}^{2}\right)=$ $(0.46 \pm 0.46) \times 10^{-2}$. In the Standard Model, $g_{\mathrm{V} \ell}\left(M_{\mathrm{Z}}^{2}\right) / g_{\mathrm{A} \ell}\left(M_{\mathrm{Z}}^{2}\right)=1-4 \sin ^{2} \theta_{\mathrm{W}}\left(M_{\mathrm{Z}}^{2}\right)$ The value obtained from $A_{\mathrm{FB}}$ agrees, within its relatively large error, with the value which follows from the determination of $\sin ^{2} \theta_{\mathrm{W}}\left(M_{\mathrm{Z}}^{2}\right)$ from $\Gamma_{\ell \ell}: g_{\mathrm{V} \ell}^{2}\left(M_{\mathrm{Z}}^{2}\right) / g_{\mathrm{A} \ell}^{2}\left(M_{\mathrm{Z}}^{2}\right)=$ $(0.70 \pm 0.19) \times 10^{-2}$.

9.3 Comparison with Other Experiments: $\sin ^{2} \theta_{\mathrm{W}}\left(M_{\mathrm{Z}}^{2}\right)$ and Limits on the Top Mass

Our measurements can be compared with the determination of the mass ratio $\frac{M_{\mathrm{W}}}{M_{\mathrm{Z}}}$ obtained by combining the results of the following experiments:

- The UA2 measurement [28] of the ratio $\frac{M_{\mathrm{W}}}{M_{\mathrm{Z}}}=0.8831 \pm 0.0055$

- The CDF measurement [29] of the $\mathrm{W}$ mass $M_{\mathrm{W}}=79.83 \pm 0.44 \mathrm{GeV}$

- The determination of $1-\left(\frac{M_{\mathrm{W}}}{M_{\mathrm{z}}}\right)^{2}=0.231 \pm 0.006$ from CDHS and CHARM results $[30]$ on neutrino-nucleon scattering

which give $\frac{M_{\mathrm{W}}}{\bar{M}_{\mathrm{Z}}}=0.8777 \pm .0027$. The constraints on the weak mixing angle and the top mass from our results on $M_{Z}$ and $\Gamma_{\ell}$ are shown in Fig. 28 together with the limits on $\sin ^{2} \theta_{\mathrm{W}}\left(M_{\mathrm{Z}}^{2}\right)$ from the mass ratio. This figure is drawn assuming $M_{\mathrm{Higgs}}=200 \mathrm{GeV}$. The width of the bands represent the experimental errors. For a given top mass, the angle is given most precisely by the $\mathrm{Z}$ mass, the remaining uncertainty is mainly due to the unknown Higgs mass. The value of $\sin ^{2} \theta_{\mathrm{W}}\left(M_{\mathrm{Z}}^{2}\right)$ which follows from our measurement of the $\mathrm{Z}$ mass using formula 6 is:

$$
\sin ^{2} \theta_{\mathrm{W}}\left(M_{\mathrm{Z}}^{2}\right)=0.2316 \pm 0.0003+0.0024\left(1-{\frac{M_{\mathrm{top}}}{150 \mathrm{GeV}}}^{2}\right)+0.0010 \log _{10} \frac{M_{\mathrm{Higgs}}}{200 \mathrm{GeV}}
$$


Figure 28: Regions in the $\sin ^{2} \theta_{\mathrm{W}}\left(M_{\mathrm{Z}}^{2}\right) \quad$ versus $M_{\text {top }}$ plane constrained by $M_{\mathrm{Z}}, \Gamma_{\ell}$ and $\frac{M_{\mathrm{W}}}{M_{\mathrm{Z}}}$ assuming $M_{\text {Higgs }}=200 \mathrm{GeV}$

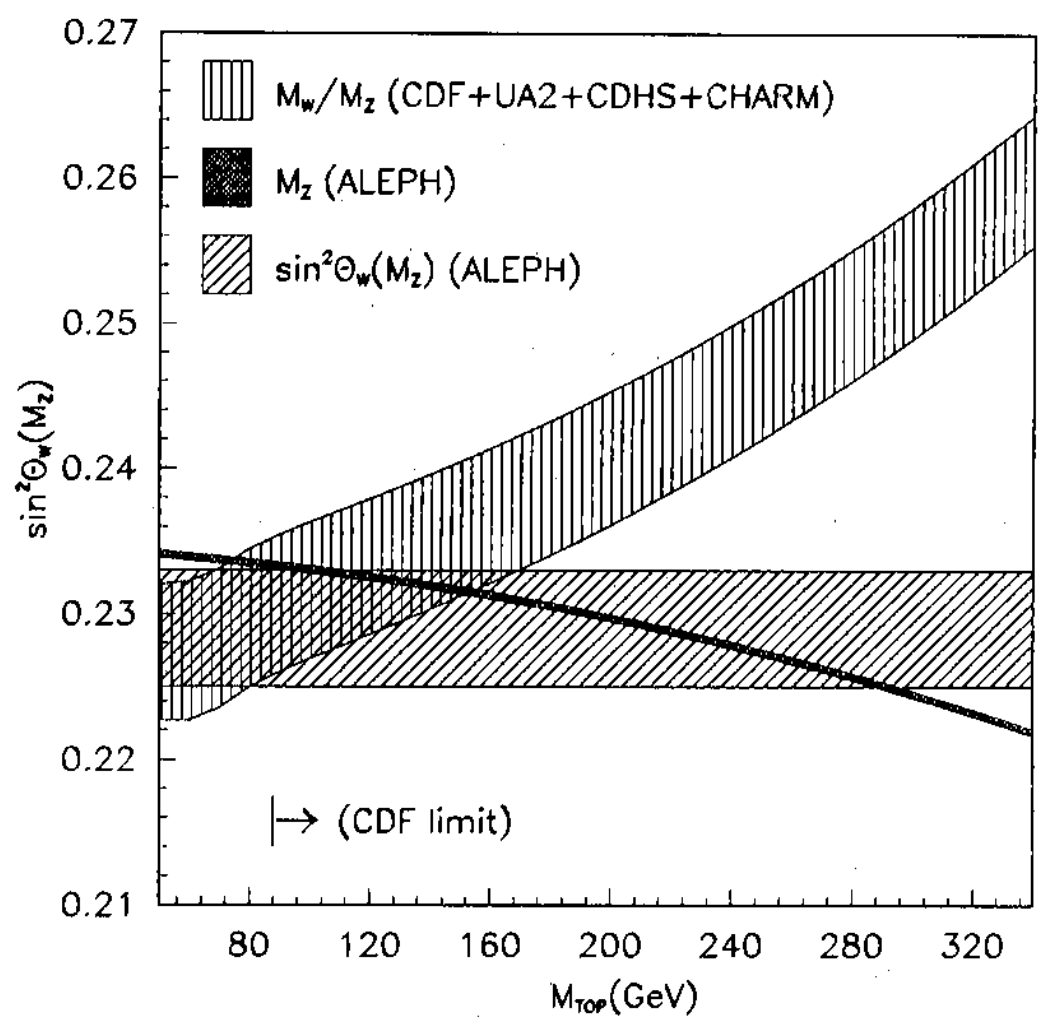

Combining this result with the mass ratio, one obtains a precise determination of $\sin ^{2} \theta_{\mathrm{W}}\left(M_{\mathrm{Z}}^{2}\right)$ :

$$
\sin ^{2} \theta_{\mathrm{W}}\left(M_{\mathrm{Z}}^{2}\right)=0.2326 \pm 0.0011 \pm 0.0005_{M_{\text {Higg }}}
$$

where the second error shows the uncertainty due to the Higgs mass ranging from 41 [31] to $1000 \mathrm{GeV}$.

The various measurements are consistent with a top mass

$$
M_{\mathrm{top}}=120 \pm 40 \pm 3_{M_{\mathrm{z}}} \pm 1_{\alpha_{\mathrm{s}}} \pm 20_{M_{\text {Higg. }}} \mathrm{GeV}
$$

where the different contributions to the error are shown separately.

\section{Conclusion}

From the data collected by ALEPH corresponding to an integrated luminosity of $2.5 \mathrm{pb}^{-1}$ we have measured the mass of the $\mathrm{Z}$ boson with a precision of $0.04 \%$ and its partial widths into hadrons and leptons with a precision of $1.5 \%$, obtaining:

$$
\begin{gathered}
M_{\mathrm{Z}}=\left(91.193 \pm 0.016_{\exp } \pm 0.030_{\mathrm{LEP}}\right) \mathrm{GeV} \\
\Gamma_{\mathrm{Z}}=(2497 \pm 31) \mathrm{MeV} \\
\sigma_{\text {had }}^{0}=(41.86 \pm 0.66) \mathrm{nb} \\
\Gamma_{\text {had }}=(1754 \pm 27) \mathrm{MeV} \\
\Gamma_{\ell \ell}=(84.3 \pm 1.3) \mathrm{MeV} \\
\Gamma_{\text {inv }}=(489 \pm 24) \mathrm{MeV}
\end{gathered}
$$


The number of light neutrinos, $N_{\nu}=2.91 \pm 0.13$, has been determined from the measured hadronic peak cross section and the ratio $\Gamma_{\text {had }} / \Gamma_{\ell}$ assuming the Standard Model value for $\Gamma_{\text {inv }} / \Gamma_{\mu}$. Using the measured value of $\Gamma_{u}$ we have determined $\sin ^{2} \theta_{\mathrm{W}}\left(M_{\mathrm{Z}}^{2}\right)=$ $0.2291 \pm 0.0040$ and with the forward-backward asymmetry of the lepton pairs in $\mathrm{Z}$ decay the vector and axial-vector weak coupling constants of leptons, $g_{\mathrm{V}}^{2}\left(M_{\mathrm{Z}}^{2}\right)=$ $(0.12 \pm 0.12) \times 10^{-2}$ and $g_{\mathrm{A}}^{2}\left(M_{\mathrm{Z}}^{2}\right)=0.2528 \pm 0.0040$ have been determined

All these results, both in the hadronic and leptonic decays, are in agreement with the Standard Model with a large top mass. They are also in good agreement with our previous measurements [1] and the measurements of the other LEP and SLC experiments [2].

\section{Acknowledgements}

It is a pleasure to thank our colleagues from the LEP division for the operation of the machine, the precise information of the absolute energy, and their continuing cooperation with us. We thank also the engineers and technicians in all our home institutes for their contribution to ALEPH's success. Those of us from non-member countries thank CERN for its hospitality. 


\section{References}

[1] D. Decamp et al. (ALEPH Collaboration): Phys. Lett. B231(1989)519, Phys. Lett. B234(1990)399, and Phys. Lett. B235(1990)339.

[2] G. S. Abrams et al. (MARK II Collaboration): Phys. Rev. Lett.63 (1989)724, G. S. Abrams et al. (MARK II Collaboration): Phys. Rev. Lett.63 (1989)2173, B. Adeva et al. (L3 Collaboration): Phys. Lett. B231(1989)509, M.Z. Akrawy et al. (OPAL Collaboration): Phys. Lett. B231(1989)530, P. Aarnio et al. (DELPHI Collaboration): Phys. Lett. B231(1989)539,

B. Adeva et al. (L3 Collaboration): Phys. Lett. B236(1990)109, B. Adeva et al. (L3 Collaboration): Phys. Lett. B237(1990)136, M.Z. Akrawy et al. (OPAL Collaboration): Phys. Lett. B240(1990)497, B. Adeva et al. (L3 Collaboration): Phys. Lett. B238(1990)122, P. Aarnio et al. (DELPHI Collaboration): Phys. Lett. B241(1990)425, P. Abreu et al. (DELPHI Collaboration): Phys. Lett. B241(1990)435, M.Z. Akrawy et al. (OPAL Collaboration): "Analysis of $Z^{\circ}$ couplings to Charged Leptons" , CERN-EP/90-81 (1990).

[3] "ALEPH - a Detector for Electron-Positron Annihilation at LEP", submitted to Nucl. Instr. and Meth. in Physics Research.

[4] F.A. Berends and R. Kleiss, Nucl.Phys. B228(1983)737, M. Böhm, A. Denner and W. Hollik, Nucl.Phys. B304(1988)687, F.A. Berends, R. Kleiss and W. Hollik, Nucl.Phys. B304(1988)712, and Computer program BABAMC, courtesy of R. Kleiss.

[5] The corresponding cross section published in our previous paper [1] was $0.7 \%$ larger due to an inadequate hadron vacuum polarization correction and a slightly different acceptance definition.

[6] H. Burkhardt et al.: Z. Phys. C43 (1989)497.

[7] D.Y. Bardin et al.: Monte Carlo group in "Proceedings of the Workshop on Z Physics at LEP", CERN Report 89-08.

[8] D. Decamp et al. (ALEPH Collaboration): Phys. Lett. B234(1990)209.

[9] M. Bengtsson and T. Sjöstrand, Phys. Lett. B185(1987)435 and

G. Marchesini and B.R. Webber, Nucl. Phys. B310(1988)461.

[10] M. Greco, Phys. Lett. B177 (1986) 97 and

M. Greco, Riv.Nuovo Cimento, 11(1988)1.

[11] M. Caffo, E. Remiddi, and F. Semaria in "Proceedings of Workshop on Z Physics at LEP", CERN Report 89-08 (1989)Vol.I,171.

[12] S. Jadach and Z. Wạs, Comp.Phys. Commun. 36(1985) 191; and Monte Carlo Group in "Proceedings of the Workshop on Z Physics at LEP", CERN Report 89-08 (1989)Vol.III.

[13] A. Borelli et al., Nucl. Phys. B333 (1990)357. 
[14] F.A. Berends et al.: Z Line Shape group in "Proceedings of the Workshop of Z Physics at LEP", CERN Report 89-08 Vol.I,89.

[15] F.A. Berends, G. Burgers and W.L. van Neerven, Nucl.Phys. B297(1988)429 and Nucl.Phys. B304(1988)921; computer program ZAPP, courtesy of G. Burgers.

[16] M. Martinez et al.: "Model Independent Fitting to the Z Line Shape", to be submitted to Phys. Lett. B.

[17] E.A. Kuraev, V.S. Fadin, Sov.J.Nucl.Phys. 41(1985)466, G. Altarelli, G. Martinelli, in "Physics at LEP", CERN 86-02 (1986) Vol. I,47, O. Nicrosini, L. Trentadue, Phys. Lett. B196(1987)551.

[18] A. Hofmann, private communication and LEP note to appear.

[19] M. Consoli, W. Hollik and F. Jegerlehner,in "Proceedings of the Workshop of Z Physics at LEP", CERN Report 89-08 (1989)

[20] D.C. Kennedy and B.W. Lynn, SLAC Pub 4039 (1986), Nucl. Phys. B322(1989)1.

[21] G. Montagna et al.: Phys. Lett. B231(1989)492 and

S. Jadach and Z. Wass, MPI-PAE/PTH 33/89,34/89(1989) and

D. Bardin et al.,Phys. Lett. B206(1988)539; computer program ZBIZON, courtesy of D. Bardin et al..

[22] K. Abe et al., Phys. Rev. Lett. 62 (1989)1709.

[23] J. Dorenbosch et al. (CHARM Collaboration): Z. Phys. C41 (1989) 567.

[24] R.C. Allen et al, Phys. Rev. Lett. 64 (1990) 1330.

[25] G.D'Agostini,W.deBoer and G.Grindhammer, Phys. Lett. B229(1989)160.

[26] W.J. Marciano and A. Sirlin, Phys. Rev. Lett. 46 (1981) 163.

[27] $\kappa$ has been computed using the program EXPOSTAR,

D.C. Kennedy, et al.: Nucl. Phys. B321 (1989)83.

[28] J. Alitti et al. (UA2 Collaboration): Phys. Lett. B241 (1990)150.

[29] P. Shalbach (CDF Collaboration): proceedings of the APS conference, Washington DC, April 90.

[30] H. Abramowicz et al. (CDHS Collaboration): Phys. Rev. Lett. 57 (1986)298, and A. Blondel et al., Z. Phys. C45 (1990) 361.

J.V. Allaby et al. (CHARM Collaboration): Phys. Lett B177 (1986)446, and Z. Phys. C36 (1987)611.

[31] D. Decamp et al. (ALEPH Collaboration): "Searches for the Standard Higgs Boson", CERN/PPE 90-101 (1990). 